\title{
Reduced-Complexity Soft-Decision Multiple-Symbol Differential Sphere Detection
}

\author{
Chao Xu, Member, IEEE, Xin Zuo, Soon Xin Ng, Senior Member, IEEE, \\ Robert G. Maunder, Senior Member, IEEE, and Lajos Hanzo, Fellow, IEEE
}

\begin{abstract}
Unlike a generic PSK/QAM detector, which may visit a constellation diagram only once, a depth-first Sphere Decoder (SD) has to re-visit the same constellation diagram multiple times. Therefore, in order to prevent the SD from repeating the detection operations, the Schnorr-Euchner search strategy of Schnorr and Euchner may be invoked for optimizing the nodes' search-order, where the ideal case is for the SD to visit the constellation nodes in a zigzag fashion. However, when the hard-decision MultipleSymbol Differential Sphere Detection (MSDSD) of Lampe et al. is invoked for using multiple receive antennas $N_{R} \geq 1$, the SchnorrEuchner search strategy has to visit and sort all the MPSK constellation points. A similar situation is encountered for the softdecision MSDSD of Pauli et al., when the a priori LLRs gleaned from the channel decoder are taken into account. In order to tackle these open problems, in this paper, we propose a correlation process for the hard-decision MSDSD of Lampe $e t$ al. and a reducedcomplexity design for the soft-decision MSDSD of Pauli et al., so that the Schnorr-Euchner search strategy always opts for visiting the MPSK constellation points in a zigzag fashion. Our simulation results demonstrate that a substantial complexity reduction is achieved by our reduced-complexity design without imposing any performance loss. Explicitly, up to $88.7 \%$ complexity reduction is attained for MSDSD $\left(N_{w}=4\right)$ aided D16PSK. This complexity reduction is quite substantial, especially when the MSDSD is invoked several times during turbo detection. Furthermore, in order to offer an improved solution and a comprehensive study for the soft-decision MSDSD, we also propose to modify the output of the SD to harmonize its operation with the near-optimum ApproxLog-MAP. Then the important subject of coherent versus noncoherent is discussed in the context of coded systems, which suggests that MSDSD aided DPSK is an eminently suitable candidate for turbo detection assisted coded systems operating at high Doppler frequencies.
\end{abstract}

Index Terms-Multiple-symbol differential sphere detection, DPSK, sphere decoding, Schnorr-Euchner search strategy, turbo detection, soft-decision, coherent versus noncoherent.

Manuscript received December 26, 2014; revised May 5, 2015; accepted July 4, 2015. The financial support of the European Research Council's Advanced Fellow Grant is gratefully acknowledged. The associate editor coordinating the review of this paper and approving it for publication was G. Bauch

The authors are with the School of Electronics and Computer Science, University of Southampton, Southampton SO17 1BJ, U.K. (e-mail: cx1g08@ecs. soton.ac.uk; xz3g08@ecs.soton.ac.uk; sxn@ecs.soton.ac.uk; rm@ecs.soton. ac.uk; lh@ecs.soton.ac.uk).

Color versions of one or more of the figures in this paper are available online at http://ieeexplore.ieee.org.

Digital Object Identifier 10.1109/TCOMM.2015.2456177

\section{INTRODUCTION}

$\mathbf{T}$ HE history of Differential Phase Shift Keying (DPSK) dates back to Lawton's classic work [4], [5] in 1959-1960, where the effect of false carrier-phase locking is mitigated by the low-complexity Conventional Differential Detection (CDD). More explicitly, the DPSK transmitter modulates the datacarrying symbols onto the phase changes between consecutive transmitted symbols, so that the CDD may recover the source information by observing the phase change between every pair of consecutive received samples. However, it was demonstrated by Cahn [6] in 1959 that the CDD-aided DPSK scheme suffers from a $3 \mathrm{~dB}$ performance penalty compared to its coherent counterpart. Moreover, it was discovered by Bello and Nelin [7] in 1962 that an irreducible error floor occurs for DPSK, when the CDD, which was originally designed for AWGN channels, is directly employed in rapidly fluctuating fading channels. In order to mitigate this problem, the Multiple-Symbol Differential Detection (MSDD) philosophy was proposed by Divsalar and Simon [8] in 1990 for DPSK invoked in AWGN channels and by Ho as well as Fung [9] in 1992 for Rayleigh fading channels. More explicitly, the MSDD extends the CDD's observation window width of $N_{w}=2$ to $N_{w} \geq 2$, where a total number of $\left(N_{w}-1\right)$ data-carrying symbols are jointly detected. The price paid is that the MSDD complexity grows exponentially with $\left(N_{w}-1\right)$. In order to reduce the MSDD complexity, a reduced-complexity algorithm was conceived for MSDD operating in AWGN channels by Mackenthun [10] in 1994, which may also be invoked for slowly-fading channels exhibiting a near-constant envelope for a block of signal transmission. As a closely related result, it was demonstrated by Cavers [11] in 1991 that accurate channel estimation relying on the classic Pilot Symbol Assisted Modulation (PSAM) may become especially challenging, when the normalized Doppler frequency is increased. Therefore, as an attractive alternative to coherent receivers, it is essentially important to implement MSDD in rapidly fluctuating fading channels at an affordable complexity.

Another low-complexity design alternative, namely the Decision-Feedback Differential Detection (DFDD) was originally proposed for AWGN channels by Leib and Pasupathy [12] in 1988. Then in 1995 it was confirmed by Leib [13] that the DFDD of [12] is equivalent to the MSDD of [8] operating in decision-feedback mode. The DFDD design was further extended to Rayleigh fading channels by Schober $e t$ al. [14] in 1999. However, the DFDD inevitably imposes a performance loss due to its inherent error-propagation problem. In order to retain the optimum MSDD performance, the state-of-the-art 
Multiple-Symbol Differential Sphere Detection (MSDSD) was proposed by Lampe et al. [2] in 2005, where the MSDD is facilitated by invoking the Sphere Decoder (SD) [15]. Furthermore, inspired by the revolutionary Turbo Code (TC) concept [16] and the generalization of the "turbo principle" [17], the softdecision MSDSD was conceived for DPSK by Pauli et al. [3] in 2006, so that the MSDSD may be invoked in turbo detection for the sake of approaching the full capacity potential of the DPSK systems.

Although the MSDD complexity may be effectively reduced by the SD, it was demonstrated by Jalden and Ottersten [18] in 2005 that the SD complexity still remains an exponential function at low SNRs. Later, Hassibi and Vikalo [19], [20] demonstrated that the expected SD complexity invoking the Fincke-Pohst enumeration strategy [21] obeyed a polynomial function. As an alternative, Kyrillidis and Karystinos [22] recently proposed a new algorithm that aimed for maximizing the Rayleigh quotient of PSK sequence detection in 2014, where the complexity was a fixed polynomial function at all SNRs. Nonetheless, further research efforts are required for enhancing this algorithm [22] in soft-decision-aided turbo detection assisted coded systems. Therefore, in this paper, we focus our attention on the MSDSD solutions [2], [3].

The classic MSDSD aided DPSK [2] was first invoked for a single receive antenna $\left(N_{R}=1\right)$. Since the recent developments in the millimeter-wave band [23], [24] facilitate the employment of a large number of antennas, especially at the Base Station (BS) [25], [26], DPSK systems relying on multiple receive antennas $N_{R} \geq 1$ may be preferred. However, when the harddecision MSDSD of [2] is invoked for $N_{R} \geq 1$, the SchnorrEuchner search strategy has to visit and sort all the MPSK constellation points. The similar situation is encountered for the soft-decision MSDSD of [3], when the a priori LLRs gleaned from the channel decoder are taken into account. Against this background, we offer the following novel contributions in this paper:

1) We propose to introduce a low-complexity correlation operation into the hard-decision MSDSD aided DPSK scheme employing an arbitrary number of receive antennas, so that the SD may visit the constellation points in a zigzag fashion, which is similar to the scenario of $N_{R}=1 \mathrm{in}$ [2].

2) It was shown in [27], [28] that substantial complexity reduction may be attained by exploring the symmetry of the Gray-labelled MPSK constellation. Against this background, we propose a reduced-complexity SchnorrEuchner search strategy for the soft-decision MSDSD of [3] employing an arbitrary number of received antennas. The proposed soft-decision MSDSD algorithm may visit a reduced number of nodes and hence achieve a substantial complexity reduction without imposing any performance loss.

Moreover, in order to offer an improved solution and a comprehensive study for the soft-decision MSDSD, the following novelties are also offered in this paper:

3) The soft-decision MSDSD proposed in [3] invokes the suboptimal Max-Log-MAP algorithm, where the SD produces only the optimum candidate. Against this background, we additionally propose to modify the output of the SD, where multiple candidates may be produced so that the nearoptimum Approx-Log-MAP may be implemented.

4) Furthermore, the important subject of coherent versus noncoherent detection is discussed for coded systems. Our simulation results suggest that compared to the coherent MPSK relying on realistic channel estimation, the DPSK schemes employing MSDSD may be deemed as a more suitable candidate for turbo detection aided coded systems operating at high Doppler frequencies.

The rest of this paper is organized as follows. The harddecision MSDSD of [2] is extended to the case of $N_{R} \geq 1$ in Section II, where the correlation operation is introduced. The soft-decision MSDSD of [3] is introduced in Section III, and then our reduced-complexity soft-decision MSDSD is proposed in Section IV. Furthermore, the near-optimum Approx-LogMAP for the soft-decision MSDSD is proposed in Section V. We provide simulation results in Section VI, where the coherent versus noncoherent discussion is offered, and our conclusions are given in Section VII. Finally, in Appendix, we present the classic SD aided V-BLAST employing MPSK [29]-[34] in the same form as the MSDSD aided DPSK, so that the proposed reducedcomplexity Schnorr-Euchner search strategy and the ApproxLog-MAP solution may be applied to the V-BLAST detection.

The following notations are used throughout the paper. The operations $(\cdot)^{*}$ and $(\cdot)^{H}$ denote the conjugate of a complex number and the Hermitian transpose of a complex matrix, respectively. The notations $\ln (\cdot)$ and $\exp (\cdot)$ refer to natural logarithm and natural exponential functions, respectively. The notations $p(\cdot)$ and $\mathrm{E}(\cdot)$ denote the probability and the expectation, respectively. The operation $\otimes$ represents the Kronecker product. The notation $\operatorname{rvec}(\mathbf{A})$ forms a row vector by taking the rows of matrix A one-by-one, while Toeplitz(a) refers to the symmetric Toeplitz matrix generated from the vector a. Moreover, the notations $\Re(\cdot)$ and $\Im(\cdot)$ take the real part and the imaginary part of a complex number, respectively.

\section{HARD-DECISION MSDSD}

For an $M$-ary DPSK scheme, the transmitter firstly maps BPS $=\log _{2} M$ source bits $\left\{b_{k}\right\}_{k=1}^{\mathrm{BPS}}$ to an MPSK symbol $x^{m}=$ $\exp \left(j \frac{2 \pi}{M} \check{m}\right)$, where the phasor index $m=\operatorname{bin} 2 \operatorname{dec}\left(b_{1} \cdots b_{\mathrm{BPS}}\right)$ is the Gray-coded index $\check{m}$. Following this, the differential encoding may be performed as:

$$
s_{n}=x_{n-1} s_{n-1} .
$$

For a Single-Input Multiple-Output (SIMO) system, the signal received by the $N_{R}$ receive antennas may be expressed as:

$$
\mathbf{Y}_{n}=s_{n} \mathbf{H}_{n}+\mathbf{V}_{n},
$$

where the $\left(1 \times N_{R}\right)$-element vectors $\mathbf{Y}_{n}, \mathbf{H}_{n}$ and $\mathbf{V}_{n}$ refer to the received signal vector, the Rayleigh fading vector and the AWGN vector which has a zero mean and a variance of $N_{0}$, respectively. Furthermore, the $N_{w}$ observations of (2) may be modelled by MSDD as [9], [35]:

$$
\mathbf{Y}=\mathbf{S H}+\mathbf{V},
$$


where we drop the time index $n$, so that the matrices in (3) are given by $\mathbf{Y}=\left[\mathbf{Y}_{N_{w}}^{T}, \cdots, \mathbf{Y}_{1}^{T}\right]^{T}, \mathbf{S}=\operatorname{diag}\left\{\left[s_{N_{w}}, \cdots, s_{1}\right]\right\}, \mathbf{H}=$ $\left[\mathbf{H}_{N_{w}}^{T}, \cdots, \mathbf{H}_{1}^{T}\right]^{T}$ and $\mathbf{V}=\left[\mathbf{V}_{N_{w}}^{T}, \cdots, \mathbf{V}_{1}^{T}\right]^{T}$. We note that the matrices $\mathbf{Y}, \mathbf{H}$ and $\mathbf{V}$ are of size $\left(N_{w} \times N_{R}\right)$, while $\mathbf{S}$ has $\left(N_{w} \times N_{w}\right)$ elements. Furthermore, since the first transmitted symbol $s_{1}$ in $\mathbf{S}$ is a common phase rotation of the following symbols $\left\{s_{t}\right\}_{t=2}^{N_{w}}$, the MSDD's received signal model of (3) may be rewritten as ${ }^{1}$ :

$$
\mathbf{Y}=\overline{\mathbf{S}} \overline{\mathbf{H}}+\mathbf{V}
$$

where the $v^{\text {th }}$ diagonal element in $\overline{\mathbf{S}}$ is given by $\bar{s}_{v}=s_{v} \cdot s_{1}^{*}$, which leads to $\bar{s}_{1}=1$ and $\bar{s}_{v}=x_{v-1} \bar{s}_{v-1}=\prod_{t=1}^{v-1} x_{t}$ for $v>1$, while the $v^{\text {th }}$ row in $\overline{\mathbf{H}}$ is given by $\overline{\mathbf{H}}_{v}=s_{1} \mathbf{H}_{v}$. The MSDD aims for maximizing the following a posteriori probability:

$$
p\left(\overline{\mathbf{S}}^{i} \mid \mathbf{Y}\right)=\frac{p\left(\mathbf{Y} \mid \overline{\mathbf{S}}^{i}\right) p\left(\overline{\mathbf{S}}^{i}\right)}{\sum_{\forall \overline{\mathbf{S}} \mathbf{j}} p\left(\mathbf{Y} \mid \overline{\mathbf{S}}^{j}\right) p\left(\overline{\mathbf{S}^{j}}\right)},
$$

where the a priori probability $p\left(\overline{\mathbf{S}}^{i}\right)$ may be assumed to be a constant of $\frac{1}{M^{\left(N_{W}-1\right)}}$ for the equiprobable candidates $\left\{\overline{\mathbf{S}}_{i}^{i}\right\}_{i=0}^{M^{\left(N_{w}-1\right)}-1}$. Furthermore, the conditional probability $p\left(\mathbf{Y} \mid \overline{\mathbf{S}}^{i}\right)$ in (5) may be expressed as:

$$
p\left(\mathbf{Y} \mid \overline{\mathbf{S}}^{i}\right)=\frac{\exp \left\{-\operatorname{rvec}(\mathbf{Y}) \cdot \mathbf{R}_{Y Y}^{-1} \cdot[\operatorname{rvec}(\mathbf{Y})]^{H}\right\}}{\pi^{N_{R} N_{w}} \operatorname{det}\left(\mathbf{R}_{Y Y}\right)},
$$

where the equivalent signal model is given by $\operatorname{rvec}(\mathbf{Y})=$ $\operatorname{rvec}(\overline{\mathbf{H}}) \cdot\left(\overline{\mathbf{S}} \otimes \mathbf{I}_{N_{R}}\right)+\operatorname{rvec}(\mathbf{V})$. As a result, the correlation matrix in (6) may be formulated as:

$$
\mathbf{R}_{Y Y}=\mathrm{E}\left\{[\operatorname{rvec}(\mathbf{Y})]^{H} \cdot \operatorname{rvec}(\mathbf{Y})\right\}=\left[\left(\overline{\mathbf{S}}^{i}\right)^{H} \mathbf{C} \overline{\mathbf{S}}^{i}\right] \otimes \mathbf{I}_{N_{R}} .
$$

More explicitly, the fading correlation matrix is given by $\mathbf{R}_{\bar{H} \bar{H}}=\mathrm{E}\left\{[\operatorname{rvec}(\overline{\mathbf{H}})]^{H} \cdot \operatorname{rvec}(\overline{\mathbf{H}})\right\}=\mathbf{R}_{h h} \otimes \mathbf{I}_{N_{R}}$, where the fading characteristic matrix is given by $\mathbf{R}_{h h}=\operatorname{Toeplitz}\left(\left[\rho_{0}, \rho_{1}, \cdots\right.\right.$, $\left.\left.\rho_{N_{w}-1}\right]\right)$. According to the Clarke model [36], the fading autocorrelation is given by $\left\{\rho_{v}=J_{0}\left(2 \pi f_{d} \cdot v\right)\right\}_{v=0}^{N_{w}-1}$, where $J_{0}(\cdot)$ is the zero-order Bessel function of the first kind, while $f_{d}$ denotes the normalized Doppler frequency. Moreover, the AWGN correlation matrix is given by $\mathbf{R}_{V V}=\mathrm{E}\left\{[\operatorname{rvec}(\mathbf{V})]^{H} \cdot \operatorname{rvec}(\mathbf{V})\right\}=$ $\mathbf{R}_{v v} \otimes \mathbf{I}_{N_{R}}$, where the AWGN characteristic matrix is simply given by $\mathbf{R}_{v v}=N_{0} \mathbf{I}_{N_{w}}$. As a result, the channel characteristic matrix in (7) is given by $\mathbf{C}=\mathbf{R}_{h h}+\mathbf{R}_{v v}$. The transmission matrix $\overline{\mathbf{S}}$ of (3) is a unitary matrix, hence the determinant term in (6) is a constant of $\operatorname{det}\left(\mathbf{R}_{Y Y}\right)=\left\{\operatorname{det}\left[\left(\overline{\mathbf{S}}^{i}\right)^{H} \cdot \operatorname{det}(\mathbf{C})\right.\right.$. $\left.\left.\operatorname{det}\left(\overline{\mathbf{S}}^{i}\right)\right]\right\}^{N_{R}}=\operatorname{det}(\mathbf{C})^{N_{R}}$. In summary, the MSDD that maximizes the a posteriori probability of (5) may be formulated as:

$$
\hat{\mathbf{S}}=\arg \min _{\forall \overline{\mathbf{S}}^{i}}\left\|\mathbf{L}\left(\overline{\mathbf{S}}^{i}\right)^{H} \mathbf{Y}\right\|^{2},
$$

where $\mathbf{L}$ is a lower triangular matrix obtained from the Cholesky decomposition of $\mathbf{C}^{-1}=\mathbf{L L}^{H}$.

\footnotetext{
${ }^{1}$ We note that $\mathbf{Y}=\left[\mathbf{Y}_{N_{w}}^{T}, \cdots, \mathbf{Y}_{1}^{T}\right]^{T}$ in (3) stores the received signal vectors in a reverse order compared to $\mathbf{Y}=\left[\mathbf{Y}_{1}^{T}, \cdots, \mathbf{Y}_{N_{w}}^{T}\right]^{T}$ seen in [2], [3], so that the MSDSD may detect the transmitted symbols according to their differential encoding order of $\bar{s}_{v}=x_{v-1} \bar{s}_{v-1}$, instead of detecting them backwards as $\bar{s}_{v}=x_{v}^{*} \bar{s}_{v+1}$.
}

In order to facilitate $\mathrm{SD},{ }^{2}$ the MSDD metric of (8) may be revised to the Euclidean Distance (ED) as:

$$
\left\|\mathbf{L}^{H} \overline{\mathbf{S}}^{H} \mathbf{Y}\right\|^{2}=\sum_{v=1}^{N_{w}}\left\|\sum_{t=1}^{v} \bar{s}_{t}^{*} \mathbf{U}_{t, v}\right\|^{2} \leq R^{2},
$$

where $R$ denotes the SD's sphere radius. The vectors $\left\{\left\{\mathbf{U}_{t, v}=\right.\right.$ $\left.\left.l_{N_{w}-t+1, N_{w}-v+1} \mathbf{Y}_{t}\right\}_{t=1}^{v}\right\}_{v=1}^{N_{w}}$ in (9) are invariant over the variables $\left\{\bar{s}_{v}\right\}_{v=2}^{N_{w}}$, where $l_{N_{w}-t+1, N_{w}-v+1}$ refers to the element taken from the $\left(N_{w}-t+1\right)$-th row and $\left(N_{w}-v+1\right)$-th column of the lower triangular matrix $\mathbf{L}$ in (8). We note that the superscript $i$ for $\overline{\mathbf{S}}^{i}$ in (8) is omitted in (9) for notational convenience. Therefore, the MSDSD's Partial Euclidean Distance (PED) based on the ED of (9) may be defined as [2]:

$$
d_{v}=\sum_{\bar{v}=1}^{v}\left\|\sum_{t=1}^{\bar{v}} \bar{s}_{t}^{*} \mathbf{U}_{t, \bar{v}}\right\|^{2}=d_{v-1}+\Delta_{v-1},
$$

and the associated PED increment as:

$$
\Delta_{v-1}=\left\|\sum_{t=1}^{v} \bar{s}_{t}^{*} \mathbf{U}_{t, v}\right\|^{2}=\left\|\bar{s}_{v-1}^{*} \mathbf{U}_{v, v}+x_{v-1}\left(\sum_{t=1}^{v-1} \bar{s}_{t}^{*} \mathbf{U}_{t, v}\right)\right\|^{2} .
$$

Observe in (10) and (11) that for a specific index $v$, all the previously tested transmitted symbols $\left\{\bar{s}_{t}\right\}_{t=1}^{v-1}$ have been decided, and the current SD search may opt for the best candidate for representing $x_{v-1}$, which is supposed to minimize $\Delta_{v-1}$.

When $N_{R}=1$ is used, it was demonstrated in [2] that the best phasor index is given by $\breve{m}_{v-1}=\left\lfloor p_{v-1}\right\rceil$, where we have $p_{v-1}=\frac{M}{2 \pi} L\left(-\bar{s}_{v-1}^{*} \mathbf{U}_{v, v} / \sum_{t=1}^{v-1} \bar{s}_{t}^{*} \mathbf{U}_{t, v}\right)$. If the phase index $\check{m}_{v-1}$ was rounded down from $p_{v-1}$, i.e. we have the condition of $p_{v-1}-\breve{m}_{v-1} \geq 0$, then the SD visits the remaining phasors in a zigzag fashion according to the steps of $\breve{m}_{v-1}=\breve{m}_{v-1}+1$, $\check{m}_{v-1}=\check{m}_{v-1}-2, \check{m}_{v-1}=\check{m}_{v-1}+3$, etc. Otherwise, the SD may visit the remaining phasors according to the steps of $\check{m}_{v-1}=$ $\check{m}_{v-1}-1, \check{m}_{v-1}=\check{m}_{v-1}+2, \check{m}_{v-1}=\check{m}_{v-1}-3$, etc. However, for the more general case of using $N_{R}>1,\left\{\left\{\mathbf{U}_{t, v}\right\}_{t=1}^{v}\right\}_{v=1}^{N_{w}}$ in (11) become vectors, hence we cannot directly obtain $p_{v-1}$. In order to mitigate this problem, we rewrite (11) as:

$$
\Delta_{v-1}=\left\|\mathbf{A}_{v-1}^{\mathrm{MSDD}}-x_{v-1} \mathbf{B}_{v-1}^{\mathrm{MSDD}}\right\|^{2}
$$

where we have $\mathbf{A}_{v-1}^{\mathrm{MSDD}}=\bar{s}_{v-1}^{*} \mathbf{U}_{v, v}$ and $\mathbf{B}_{v-1}^{\mathrm{MSDD}}=-\sum_{t=1}^{v-1} \bar{s}_{t}^{*} \mathbf{U}_{t, v}$. As a result, a simple correlation process leads us to the following decision variable:

$$
z_{v-1}=\mathbf{A}_{v-1}^{\mathrm{MSDD}}\left(\mathbf{B}_{v-1}^{\mathrm{MSDD}}\right)^{H}
$$

which may be directly used for detecting $x_{v-1}$. More explicitly, the best phasor is now given by $\check{m}_{v-1}=\left\lfloor p_{v-1}\right\rceil$, where we have $p_{v-1}=\frac{M}{2 \pi} \angle z_{v-1}$, and the Schnorr-Euchner search strategy may visit the remaining phasors in a zigzag fashion in the same way as the case of $N_{R}=1$ in [2].

\footnotetext{
${ }^{2}$ We note that the depth-first tree search strategy of [30] and the SchnorrEuchner constellation search strategy of [1] constitute the default choice for the MSDSD [2], [3], which enables the MSDSD to achieve the same detection capability as the MSDD.
} 


\section{SofT-DECISION MSDSD}

According to the a posteriori probability of (5), the optimum Log-MAP algorithm invoked by MSDD may be expressed as [37], [38]

$$
\begin{aligned}
L_{p}\left(b_{k}\right) & =\ln \frac{\sum_{\overline{\mathbf{S}}_{i} \in \overline{\mathbf{S}}_{b_{k}=1}} p\left(\overline{\mathbf{S}}^{i} \mid \mathbf{Y}\right)}{\sum_{\overline{\mathbf{S}}_{i} \in \overline{\mathbf{S}}_{b_{k}=0}} p\left(\overline{\mathbf{S}}^{i} \mid \mathbf{Y}\right)} \\
& =\ln \frac{\sum_{\overline{\mathbf{S}}^{i} \in \overline{\mathbf{S}}_{b_{k}=1}} \exp \left(d^{i}\right)}{\sum_{\overline{\mathbf{S}}_{i} \in \overline{\mathbf{S}}_{b_{k}=0}} \exp \left(d^{i}\right)}=L_{a}\left(b_{k}\right)+L_{e}\left(b_{k}\right),
\end{aligned}
$$

where $L_{p}\left(b_{k}\right), L_{e}\left(b_{k}\right)$ and $L_{a}\left(b_{k}\right)$ represent the a posteriori LLR and the extrinsic LLR produced by the MSDD as well as the a priori LLR gleaned from a channel decoder, respectively. Furthermore, $\overline{\mathbf{S}}_{b_{k}=1}$ and $\overline{\mathbf{S}}_{b_{k}=0}$ refer to the MSDD signal set $\overline{\mathbf{S}}$, when the specific bit $b_{k}$ is set to 1 and 0 , respectively. The probability metric ${ }^{3}\left\{d^{i}\right\}_{i=0}^{M^{\left(N_{w}-1\right)}-1}$ seen in (14) is given by $d^{i}=$ $-\left\|\mathbf{L}^{H}\left(\overline{\mathbf{S}}^{i}\right)^{H} \mathbf{Y}\right\|^{2}+\sum_{\bar{k}=1}^{\left(N_{w}-1\right) \mathrm{BPS}} \widetilde{b}_{\bar{k}} L_{a}\left(b_{\bar{k}}\right)$, where $\left\{\widetilde{b}_{\bar{k}}\right\}_{\bar{k}=1}^{\left(N_{w}-1\right) \mathrm{BPS}}$ denotes the bit-mapping corresponding to the MSDD candidate $\mathbf{S}^{i}$. The Log-MAP algorithm of (14) may be simplied by the low-complexity Max-Log-MAP [37] as:

$$
L_{p}\left(b_{k}\right)=\max _{\overline{\mathbf{S}}^{i} \in \overline{\mathbf{S}}_{b_{k}=1}} d^{i}-\max _{\overline{\mathbf{S}}^{i} \in \overline{\mathbf{S}}_{b_{k}=0}} d^{i} .
$$

Furthermore, in order to compensate for the sub-optimum MaxLog-MAP, the Approx-Log-MAP was introduced as [39]:

$$
L_{p}\left(b_{k}\right)=\operatorname{jac}_{\overline{\mathbf{S}}_{i} \in \overline{\mathbf{S}}_{b_{k}=1}} d^{i}-\operatorname{jac}_{\overline{\mathbf{S}}^{i} \in \overline{\mathbf{S}}_{b_{k}=0}} d^{i} .
$$

where jac denotes the Jacobian algorithm of $\operatorname{jac}\left(d^{1}, d^{2}\right)=$ $\max \left\{d^{1}, d^{2}\right\}+\Gamma\left\{\left|d^{1}-d^{2}\right|\right\}$, while the additional term of $\Gamma\left\{\left|d^{1}-d^{2}\right|\right\}$ may take into account the difference between $d^{1}$ and $d^{2}$ according to a lookup table.

The Max-Log-MAP aims for finding the maximum probability metrics, which is similar to the action of hard-decision detectors. Therefore, in order to invoke SD for the Max-LogMAP, the maximization of (15) has to be revised for the sake of minimization, while the probability metrics should be guaranteed to have positive values. As a result, the MSDD probability metric of (14) may be transformed into:

$$
d=\sum_{v=1}^{N_{w}}\left\|\sum_{t=1}^{v} \bar{s}_{t}^{*} \mathbf{U}_{t, v}\right\|^{2}-\sum_{v=2}^{N_{w}} \sum_{\bar{k}_{v}=1}^{\text {BPS }}\left[\widetilde{b}_{\bar{k}_{v}} L_{a}\left(b_{\bar{k}_{v}}\right)-\bar{C}_{a, \bar{k}_{v}}\right],
$$

where the superscript $i$ for $d^{i}$ seen in (14) is deleted for the sake of convenience, while the polarity of $d^{i}$ in (14) is altered. Furthermore, the constant $\bar{C}_{a, \bar{k}_{v}}$ in (17) was formulated as $\bar{C}_{a, \bar{k}_{v}}=$ $\ln \prod_{\bar{k}_{v}=1}^{\mathrm{BPS}}\left\{1+\exp \left[L_{a}\left(b_{\bar{k}_{v}}\right)\right]\right\}$ in [3], which was originally

\footnotetext{
${ }^{3}$ We note that all multiplicative factors of the $\exp \left(d^{i}\right)$ term are eliminated by the division operation in (14), which include the denominator $\left[\sum_{\forall \overline{\mathbf{S}} j} p\left(\mathbf{Y} \mid \overline{\mathbf{S}}^{j}\right) p\left(\overline{\mathbf{S}}^{j}\right)\right]$ of $p\left(\overline{\mathbf{S}}^{i} \mid \mathbf{Y}\right)$ in (5), the denominator $\left[\pi^{N_{R} N_{w}} \operatorname{det}\left(\mathbf{R}_{Y Y}\right)\right]$ of $p\left(\mathbf{Y} \mid \overline{\mathbf{S}}^{i}\right)$ in (6) as well as the denominator $\prod_{\bar{k}=1}^{\left(N_{w}-1\right) \operatorname{BPS}}\left\{1+\exp \left[L_{a}\left(b_{\bar{k}}\right)\right]\right\}$ in the a priori probability of $p\left(\overline{\mathbf{S}}^{i}\right)=\frac{\exp \left[\sum_{\bar{k}=1}^{\left(N_{w}-1\right) \mathrm{BPS}} \widetilde{b}_{\bar{k}} L_{a}\left(b_{\bar{k}}\right)\right]}{\prod_{\bar{k}=1}^{\left(N_{w}-1\right) \mathrm{BPS}}\left\{1+\exp \left[L_{a}\left(b_{\bar{k}}\right)\right]\right\}}$ according to the LLR definition of $L_{a}=\ln \frac{p(b=1)}{p(b=0)}$ [37], [38].
}

eliminated by the the division of the Log-MAP of (14). However, in order to avoid excessive calculations in logarithm domain, we adopt the method in [34], [40], which uses a simple operation of $\bar{C}_{a, \bar{k}_{v}}=\frac{1}{2}\left[\left|L_{a}\left(b_{\bar{k}_{v}}\right)\right|+L_{a}\left(b_{\bar{k}_{v}}\right)\right]$ to guarantee a nonnegative ED. As a result, the PED of soft-decision MSDSD may be defined as [3]:

$$
\begin{aligned}
d_{v} & =\sum_{\bar{v}=2}^{v}\left\|\sum_{t=1}^{\bar{v}} \bar{s}_{t}^{*} \mathbf{U}_{t, \bar{v}}\right\|^{2}-\sum_{\bar{v}=2}^{v} \sum_{\bar{k}_{\bar{v}}=1}^{\mathrm{BPS}}\left[\widetilde{b}_{\bar{k}_{\bar{v}}} L_{a}\left(b_{\bar{k}_{\bar{v}}}\right)-\bar{C}_{a, \bar{k}_{\bar{v}}}\right] \\
& =d_{v-1}+\Delta_{v-1},
\end{aligned}
$$

where the PED increment is given by:

$$
\begin{aligned}
\Delta_{v-1}=\| \bar{s}_{v-1}^{*} \mathbf{U}_{v, v}+x^{m} & \left(\sum_{t=1}^{v-1} \bar{s}_{t}^{*} \mathbf{U}_{t, v}\right) \|^{2} \\
& -\sum_{\bar{k}_{v}=1}^{\mathrm{BPS}}\left[\widetilde{b}_{\bar{k}_{v}} L_{a}\left(b_{\bar{k}_{v}}\right)-\bar{C}_{a, \bar{k}_{v}}\right] .
\end{aligned}
$$

As introduced in [3], the Schnorr-Euchner search strategy may search for $x_{v-1}$ according to $\Delta_{v-1}$ of (19). However, unlike the hard-decision MSDSD, the decision variable $z_{v-1}$ of (13) cannot be directly used, because the a priori information $\sum_{\bar{k}_{v}=1}^{\mathrm{BPS}}\left[\widetilde{b}_{\bar{k}_{v}} L_{a}\left(b_{\bar{k}_{v}}\right)-\bar{C}_{a, \bar{k}_{v}}\right]$ is not included in $z_{v-1}$. As a result, the conventional Schnorr-Euchner search strategy in [3] has to visit all MPSK constellation points for $x_{v-1}$ by evaluating and sorting a total of $M$ PED increment values $\Delta_{v-1}$ of (19).

The soft-decision MSDSD algorithm based on the PED of (18) may now be implemented by the "MAP-MSDSD" function in [3], which is exemplified for the cases of employing DQPSK and D8PSK in Figs. 1 and 2, respectively. In summary, the MSDSD algorithm in [3] is capable of finding both the global minimum $d_{M A P}$ as well as the optimum constellation points $\left\{\hat{x}_{v-1}\right\}_{v=2}^{N_{w}-1}$, which may be translated into the hard-bit decisions of $\left\{b_{k}^{M A P}\right\}_{k=1}^{\left(N_{w}-1\right) \mathrm{BPS}}$. In order to produce soft-bit decisions, the Max-Log-MAP algorithm of (15) may be completed as:

$$
L_{p}\left(b_{k}\right)= \begin{cases}-d_{M A P}+\bar{d}_{M A P}, & \text { if } b_{k}^{M A P}=1 \\ -\bar{d}_{M A P}+d_{M A P}, & \text { if } b_{k}^{M A P}=0\end{cases}
$$

where $\bar{d}_{M A P}$ is obtained by invoking the MSDSD again, where the search space is halved by fixing $b_{k}$ to be the flipped version of the MAP decision as $b_{k}=\bar{b}_{k}^{M A P}$. In summary, when the consecutive MSDSD windows are simply overlapped by $N_{O L}=1$ observations, the MSDSD algorithm of [3] has to be invoked once first for finding the global MAP solution $d_{M A P}$ in (20), and then it is invoked for an additional $\left(N_{w}-1\right) \log _{2} M$ number of times for finding the local MAP solutions $\bar{d}_{M A P}$ in (20), which may be referred to as the Repeated Tree Search (RTS).

Alternatively, it's recently proposed in [33], [34] that the Single Tree Search (STS) [41] may opt to invoke the SD only once for obtaining all the EDs of $d_{M A P}$ and $\bar{d}_{M A P}$, which may induce a potential performance loss. More explicitly, if the hypothesis bit-mapping arrangement for $d_{M A P}$ is updated and changed, all the counter-hypothesis bit-mapping arrangements for $\bar{d}_{M A P}$ have to be changed accordingly. As a result, the previously dismissed candidates that obey the new bit-mapping cannot be taken into account again. As a remedy, the sub-optimal 


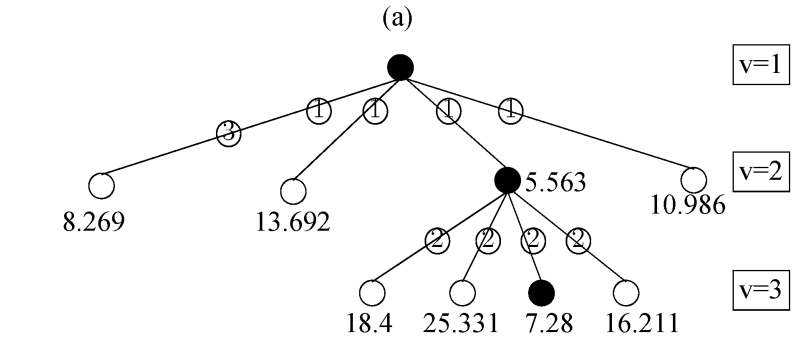

$\bigcirc \quad$ constellation points that are visited by the SD paths that are visited by the SD

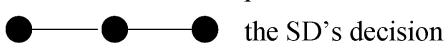

(1), (2), (3), $\cdots$ the SD's steps

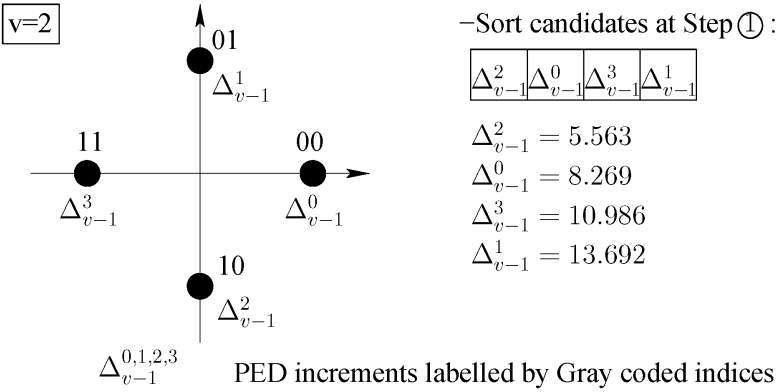

Fig. 1. Example of soft-decision MSDSD aided DQPSK, recorded at SNR $=$ $0 \mathrm{~dB}$, where we have $I_{A}=0.3, N_{R}=2$ and $N_{w}=3$. (a) Example of Soft-Decision MSDSD Conceived for DQPSK Detection (labelled with PED values). (b) Example of QPSK constellation digram visited by Soft-Decision $\mathrm{SD}$ at index $\mathrm{v}=2$.

(a)

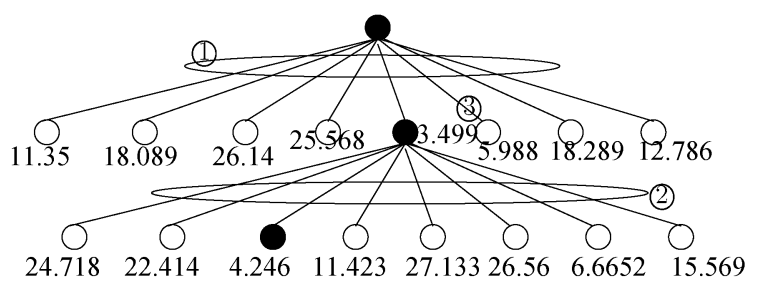

$\mathrm{v}=1$

$\mathrm{v}=2$

$\mathrm{v}=3$

(b)

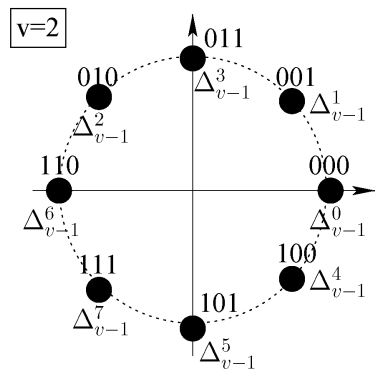

-Sort candidates at Step (1): \begin{tabular}{|l|l|l|l|l|l|l|l|}
\hline$\Delta_{v-1}^{4}$ & $\Delta_{v-1}^{5}$ & $\Delta_{v-1}^{0}$ & $\Delta_{v-1}^{7}$ & $\Delta_{v-1}^{1}$ & $\Delta_{v-1}^{6}$ & $\Delta_{v-1}^{3}$ & $\Delta_{v-1}^{2}$ \\
\hline
\end{tabular}

$\Delta_{v-1}^{4}=3.499$

$\Delta_{v-1}^{5}=5.988$

$\Delta_{v-1}^{0}=11.35$

$\Delta_{v-1}^{7}=12.786$

$\Delta_{v-1}^{1}=18.089$

$\Delta_{v-1}^{6}=18.289$

$\Delta_{v-1}^{3}=25.568$

$\Delta_{v-1}^{2}=26.14$

Fig. 2. Example of soft-decision MSDSD aided D8PSK, recorded at SNR = $3 \mathrm{~dB}$, where we have $I_{A}=0.3, N_{R}=2$ and $N_{w}=3$. (a) Example of SoftDecision MSDSD Conceived for D8PSK Detection. (b) Example of 8PSK constellation digram visited by Soft-Decision SD at index v $=2$.

detector has to invoke the LLR correction method [34] for correcting the LLR results. Against this background, the RTS is suggested in this paper. In fact, the STS's motivation of visiting a node at most once can still be accomplished by the RTS, where the previously visited nodes may be labelled so that the repeated calculations may be avoided by reading the previously evaluated PED metrics.

\section{REDUCED-COMPLEXITY SOFT-DECISION MSDSD}

It was demonstrated in Section III that the conventional Schnorr-Euchner search strategy utilized by the soft-decision MSDSD of [3] has to visit all MPSK constellation points. In this section, we opt to propose a reduced-complexity soft-decision MSDSD algorithm, where the Schnorr-Euchner search strategy may once again visit the MPSK constellation points in a zigzag fashion. More explicitly, the PED increment $\Delta_{v-1}$ of (19) may be further extended as:

$$
\begin{aligned}
& \Delta_{v-1}=\left\|\mathbf{A}_{v-1}^{\mathrm{MSDD}}-x_{v-1} \mathbf{B}_{v-1}^{\mathrm{MSDD}}\right\|^{2}-\sum_{\bar{k}_{v}=1}^{\mathrm{BPS}}\left[\widetilde{b}_{\bar{k}_{v}} L_{a}\left(b_{\bar{k}_{v}}\right)-\bar{C}_{a, \bar{k}_{v}}\right] \\
& =-2 \Re\left(\bar{x}_{v-1}^{*} \bar{z}_{v-1}\right)-\sum_{\bar{k}_{v}=1}^{\mathrm{BPS}} \widetilde{b}_{\bar{k}_{v}} L_{a}\left(b_{\bar{k}_{v}}\right)+\frac{L_{a}\left(b_{1}\right)+L_{a}\left(b_{2}\right)}{2}+\bar{C}_{v-1},
\end{aligned}
$$

where we deliberately rotate all the detected MPSK constellations (except for $\mathrm{BPSK}^{4}$ ) anti-clockwise by $(\pi / M)$ as suggested by [28], i.e. we have $\bar{x}_{v-1}=x_{v-1} \cdot \exp \left(j \frac{\pi}{M}\right)$, so that there are exactly $M / 4$ constellation points in each quadrant. Furthermore, the new decision variable $\bar{z}_{v-1}$ seen in (21) is given by:

$$
\bar{z}_{v-1}=\mathbf{A}_{v-1}^{\mathrm{MSDD}}\left(\mathbf{B}_{v-1}^{\mathrm{MSDD}}\right)^{H} \cdot \exp \left(j \frac{\pi}{M}\right),
$$

which is rotated anti-clockwise from the correlation decision variable $z_{v-1}^{M S D S D}$ of (13) by $(\pi / M)$ for detecting $\bar{x}_{v-1}$, while the constant of $\bar{C}_{v-1}$ seen in (21) is given by:

$\bar{C}_{v-1}=\left\|\mathbf{A}_{v-1}^{\mathrm{MSDD}}\right\|^{2}+\left\|\mathbf{B}_{v-1}^{\mathrm{MSDD}}\right\|^{2}+\bar{C}_{a, v-1}-\frac{L_{a}\left(b_{1}\right)+L_{a}\left(b_{2}\right)}{2}$,

and we have the constant $\bar{C}_{a, v-1}=\sum_{\bar{k}_{v}=1}^{\mathrm{BPS}} \bar{C}_{a, \bar{k}_{v}}$. We note that $\bar{C}_{v-1}$ of (23) is invariant over all the different candidates $\bar{x}_{v-1}$ in (21). As a result, comparing the $M$ candidates $\left\{x^{m}\right\}_{m=0}^{M-1}$ according to their PED increment values $\Delta_{v-1}$ of (21) is equivalent to comparing the following equivalent PED increment metric over the variables $\bar{x}_{v-1}$ as:

$$
\begin{aligned}
\bar{\Delta}_{v-1}= & -2 \Re\left(\bar{x}_{v-1}\right) \Re\left(\bar{z}_{v-1}\right)-2 \Im\left(\bar{x}_{v-1}\right) \Im\left(\bar{z}_{v-1}\right) \\
& -\sum_{\bar{k}_{v}=1}^{\mathrm{BPS}} \widetilde{b}_{\bar{k}_{v}} L_{a}\left(b_{\bar{k}_{v}}\right)+\frac{L_{a}\left(b_{1}\right)+L_{a}\left(b_{2}\right)}{2},
\end{aligned}
$$

where we have the algebraic relationship of $\Re\left(\bar{x}_{v-1}^{*} \bar{z}_{v-1}\right)=$ $\Re\left(\bar{x}_{v-1}\right) \Re\left(\bar{z}_{v-1}\right)+\Im\left(\bar{x}_{v-1}\right) \Im\left(\bar{z}_{v-1}\right)$.

Considering the rotated QPSK as an example, the four probability metrics $\left\{\bar{\Delta}_{v-1}^{m}\right\}_{m=0}^{M-1=3}$ of (24) corresponding to the $M=4$ rotated QPSK constellation points $\left\{\bar{x}^{m}= \pm \frac{1}{\sqrt{2}} \pm j \frac{1}{\sqrt{2}}\right\}_{m=0}^{M-1=3}$ may be expressed as (25), shown at the bottom of the next page.

\footnotetext{
${ }^{4}$ We note that rotating the BPSK constellation anti-clockwise by $(\pi / M)$ will only move the two BPSK constellation points from the real axis to the imaginary axis, which is not neccessary.
} 
TABLE I

PSEUdo-CODE FOR REDUCED-COMPLEXITY SOFT-DECISION MSDSD AIDED DPSK

\begin{tabular}{|c|c|c|}
\hline \multicolumn{3}{|c|}{$\begin{array}{l}\text { Function: } \quad\left[\left\{\hat{x}_{v-1}\right\}_{v=2}^{N_{w}}, d_{M A P}\right]=\text { Soft-MSDSD-RC }\left(\left\{\left\{\mathbf{U}_{t, v}\right\}_{t=1}^{v}\right\}_{v=1}^{N_{w}},\left\{\left\{L_{a}^{v-1, k}\right\}_{v w}^{N_{w}}\right\}_{k=1}^{2},\left\{\bar{C}_{a, v-1}\right\}_{v=2}^{N_{w}},\right. \\
\left.\left\{\left\{P_{v-1}^{g}\right\}_{g=0}^{M / 4-1}\right\}_{v=2}^{N_{w}}, N_{w}, R^{2}\right)\end{array}$} \\
\hline 1: $d_{1}=0$ & & //initialize PED \\
\hline //initialize the first transmitted symbol & & //initialize the first transmitted symbol \\
\hline \multicolumn{3}{|r|}{ //initialize SD search index } \\
\hline \multicolumn{3}{|c|}{ 4: (subfunction) findBest-DBPSK/findBest-DQPSK/findBest-DPSK } \\
\hline \multicolumn{3}{|l|}{ 5: loop } \\
\hline \multicolumn{3}{|c|}{ 6: $\quad d_{v}=d_{v-1}+\bar{\Delta}_{v-1}+\bar{C}_{v-1}$} \\
\hline \multicolumn{3}{|r|}{ //update candidate data symbol } \\
\hline \multicolumn{3}{|r|}{ //update candidate transmitted symbol } \\
\hline \multicolumn{3}{|c|}{ if $v \neq N_{w}$} \\
\hline \multirow{2}{*}{\multicolumn{3}{|c|}{$\begin{array}{l}v=v+1 \\
\text { (subfunction) findBest-DBPSK/findBest-DQPSK/findBest-DPSK }\end{array}$}} \\
\hline & & \\
\hline \multicolumn{3}{|l|}{ else } \\
\hline \multicolumn{3}{|r|}{ //update SD radius } \\
\hline $\multicolumn{3}{|c|}{\hat{x}_{v-1}}_{v=2}^{N_{w}^{w}}=\left\{x_{v-1}\right\}_{v=2}^{N_{w}}$ \\
\hline \multicolumn{2}{|r|}{ if $v==2$ return $\left[\left\{\hat{x}_{v-1}\right\}_{v=2}^{N_{w}}, R^{2}\right]$ and exit } & $\begin{array}{l}/ / \text { terminate } \mathrm{SD} \text { for the case of } N_{w}=2 \\
/ / \text { move down }\end{array}$ \\
\hline \multicolumn{3}{|c|}{ while $n_{v-1}==(M-1)$} \\
\hline \multicolumn{3}{|c|}{ end if } \\
\hline \multicolumn{3}{|l|}{ 22: $\quad$ else } \\
\hline \multicolumn{3}{|l|}{ 23: do } \\
\hline \multicolumn{2}{|r|}{ if $v==2$ return $\left[\left\{\hat{x}_{v-1}\right\}_{v=2}^{N_{w}}, R^{2}\right]$ and exit } & $/ /$ terminate $\mathrm{SD}$ when $v=2$ is reached \\
\hline & & $/ /$ move down \\
\hline \multicolumn{3}{|c|}{ while $n_{v-1}==(M-1)$} \\
\hline \multicolumn{2}{|r|}{ (subfunction) findNext-DBPSK/findNext-DQPSK/findNext-DPSK } & //find the next candidate for index $v$ \\
\hline \multicolumn{3}{|l|}{ 28: end if } \\
\hline 29: end loop & & \\
\hline
\end{tabular}

where we associate the real and imaginary parts of $\bar{z}_{v-1}$ with $L_{a}\left(b_{2}\right)$ and $L_{a}\left(b_{1}\right)$ respectively as:

$t_{v-1}^{R e}=\sqrt{2} \Re\left(\bar{z}_{v-1}\right)-\frac{L_{a}\left(b_{2}\right)}{2}, \quad t_{v-1}^{I m}=\sqrt{2} \Im\left(\bar{z}_{v-1}\right)-\frac{L_{a}\left(b_{1}\right)}{2}$.

After assigning the a priori LLRs to the appropriate parts of $\bar{z}_{v-1}$, the only difference between the four candidates $\left\{\bar{\Delta}_{v-1}^{m}\right\}_{m=0}^{M-1=3}$ in (25) is the polarity of $t_{v-1}^{R e}$ and $t_{v-1}^{I m}$. This allows us to directly obtain the minimum metric by simply evaluating $\bar{\Delta}_{v-1}=-\left|t_{v-1}^{R e}\right|-\left|t_{v-1}^{I m}\right|$, and then the ranking order of the rest of the candidates may be obtained by comparing $\left|t_{v-1}^{R e}\right|$ and $\left|t_{v-1}^{I m}\right|$. In more detail, if we have the condition of $\left|t_{v-1}^{R e}\right|>$ $\left|t_{v-1}^{I m}\right|$, then the SD may visit the remaining candidates in a zigzag fashion according to the steps of $\bar{\Delta}_{v-1}=-\left|t_{v-1}^{R e}\right|+\left|t_{v-1}^{I m}\right|$, $\bar{\Delta}_{v-1}=\left|t_{v-1}^{R e}\right|-\left|t_{v-1}^{I m}\right|$ and $\bar{\Delta}_{v-1}=\left|t_{v-1}^{R e}\right|+\left|t_{v-1}^{I m}\right|$. Other- wise, the remaining steps should be $\bar{\Delta}_{v-1}=\left|t_{v-1}^{R e}\right|-\left|t_{v-1}^{I m}\right|$, $\bar{\Delta}_{v-1}=-\left|t_{v-1}^{R e}\right|+\left|t_{v-1}^{I m}\right|$ and $\bar{\Delta}_{v-1}=\left|t_{v-1}^{R e}\right|+\left|t_{v-1}^{I m}\right|$. In summary, similar to the condition of $\operatorname{sign}\left(p_{v-1}-\left\lfloor p_{v-1}\right\rceil\right)$ for the hard-decision MSDSD of Section II, the soft-decision MSDSD aided DQPSK may rely on the condition of $\operatorname{sign}\left(\left|t_{v-1}^{R e}\right|-\right.$ $\left.\left|t_{v-1}^{I m}\right|\right)$ for deciding the direction of SD's zigzag path.

In more detail, the reduced-complexity soft-decision MSDSD is summarized in the form of its pseudo-code in Table I, where the simplified Schnorr-Euchner search strategy specifically tailored for DBPSK and DQPSK is given by Tables II and III, respectively. Furthermore, Fig. 3 revisits the specific example of Fig. 1, where the reduced-complexity algorithms of Tables I and III are invoked. It can be seen in Fig. 3 that the proposed reduced-complexity MSDSD exhibits a reduced number of visited nodes compared to the conventional MSDSD exemplified by Fig. 1.

$$
\begin{aligned}
& \bar{\Delta}_{v-1}^{0}=-\sqrt{2} \Re\left(\bar{z}_{v-1}\right)-\sqrt{2} \Im\left(\bar{z}_{v-1}\right)+\frac{L_{a}\left(b_{1}\right)+L_{a}\left(b_{2}\right)}{2}=-t_{v-1}^{R e}-t_{v-1}^{I m}, \\
& \bar{\Delta}_{v-1}^{1}=\sqrt{2} \Re\left(\bar{z}_{v-1}\right)-\sqrt{2} \Im\left(\bar{z}_{v-1}\right)-L_{a}\left(b_{2}\right)+\frac{L_{a}\left(b_{1}\right)+L_{a}\left(b_{2}\right)}{2}=t_{v-1}^{R e}-t_{v-1}^{I m}, \\
& \bar{\Delta}_{v-1}^{2}=-\sqrt{2} \Re\left(\bar{z}_{v-1}\right)+\sqrt{2} \Im\left(\bar{z}_{v-1}\right)-L_{a}\left(b_{1}\right)+\frac{L_{a}\left(b_{1}\right)+L_{a}\left(b_{2}\right)}{2}=-t_{v-1}^{R e}+t_{v-1}^{I m}, \\
& \bar{\Delta}_{v-1}^{3}=\sqrt{2} \Re\left(\bar{z}_{v-1}\right)+\sqrt{2} \Im\left(\bar{z}_{v-1}\right)-L_{a}\left(b_{1}\right)-L_{a}\left(b_{2}\right)+\frac{L_{a}\left(b_{1}\right)+L_{a}\left(b_{2}\right)}{2}=t_{v-1}^{R e}+t_{v-1}^{I m},
\end{aligned}
$$


TABLE III

Pseudo-Code For the Subfunctions of THE REDuCED-COMPLEXITy SofT-DeCision MSDSD OF TABLE I, WhERE DQPSK IS EMPLOYED

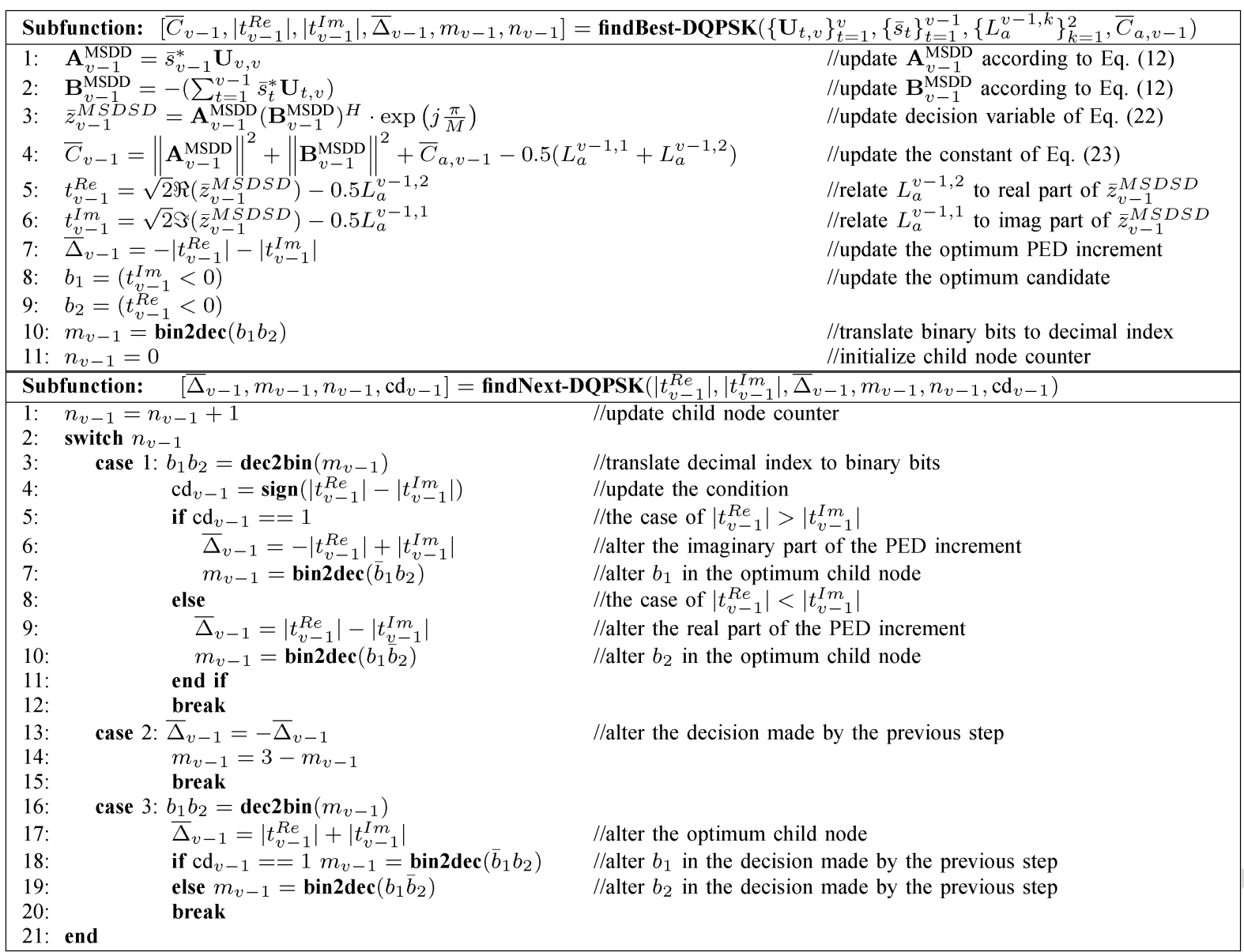

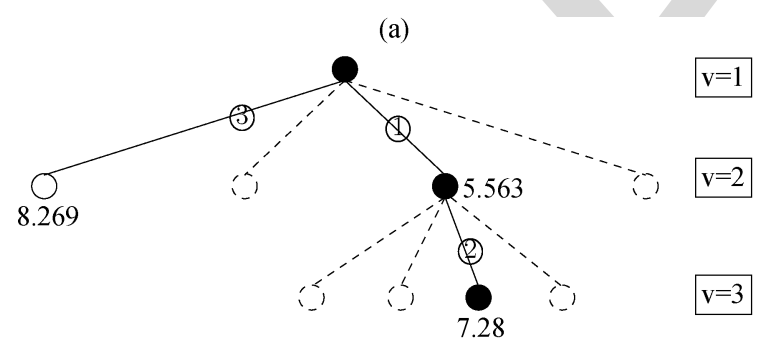

(-..., the SD paths that are avoided

because of the reduced-complexity design

(b)

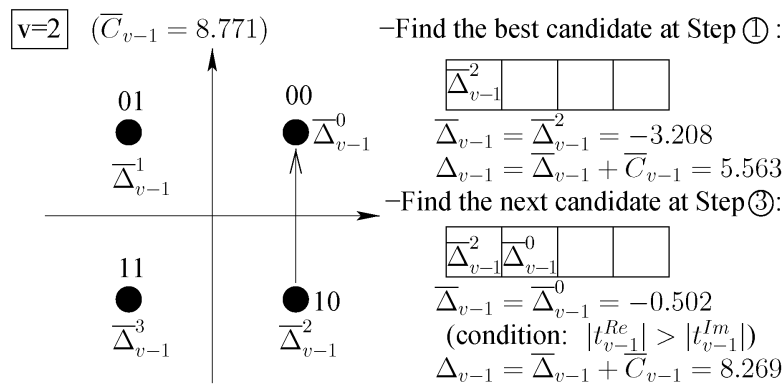

Fig. 3. Example of soft-decision MSDSD aided DQPSK, which corresponds to the example seen in Fig. 1, subject to the difference that the reducedcomplexity algorithm of Tables I and III are invoked. (a) Example of ReducedComplexity Soft-Decision MSDSD Conceived for DQPSK Detection (b) Example of QPSK constellation diagram visited by Reduced-Complexity Soft-Decision SD at index $\mathrm{v}=2$. strategy to invoke a sorting algorithm, which was represented by the "qsort" function on line F-6 in the pseudo-code algorithm table of [3]. As a result, all $M$ constellation points $\left\{x^{m}\right\}_{m=0}^{M-1}$ are ranked according to an ascending order of the PED increment values $\left\{\Delta_{v-1}^{m}\right\}_{m=0}^{M-1}$, which is explicitly exemplified by Figs. 1 and 2. There are numerous sorting algorithms that may be suitable, such as Bubble sort, Timsort, Library sort [42], [43], etc., but the average number of comparisons required by these algorithms is as high as $O(M \log M)$. By contrast, the reduced-complexity MSDSD of Tables II-IV does not require any sorting algorithms. As exemplified by Figs. 3 and 4, the proposed Schnorr-Euchner search strategy does not have to maintain the complete ranking order of constellation points, which dispenses with a considerable number of comparisons.

\section{APPROX-LOG-MAP IMPLEMENTED BY MSDSD}

The soft-decision MSDSD discussed in the Sections III and IV aims to implement the Max-Log-MAP of (15), which may impose a performance loss compared to the near-optimum ApproxLog-MAP of (16). In order to mitigate this open problem, we propose the Approx-Log-MAP solution for MSDSD as follows:

(1) Let us define the leaf nodes of a SD structure as the candidates associated with the SD index $v=N_{w}$. For example, the $M=4$ candidates visited at the SD's step of (2) in Fig. 1(a) as well as the $M=8$ candidates visited at step (2) 
(a)

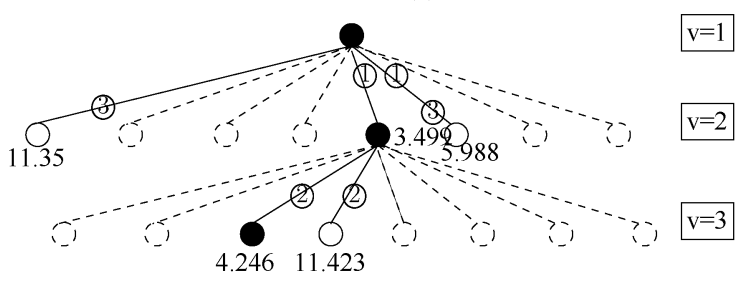

(b)

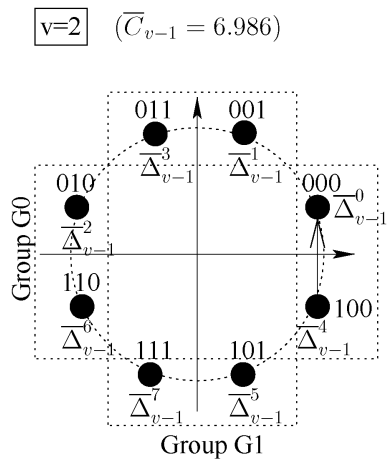

-Find the best candidate at Step (1)

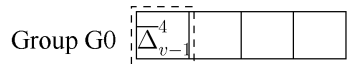

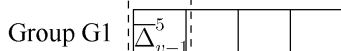

Comparison Window: $\bar{\Delta}_{v-1}^{G 0}=\bar{\Delta}_{v-1}^{4}=-3.487$ $\bar{\Delta}_{v-1}^{G 1}=\bar{\Delta}_{v-1}^{5}=-0.998$ $\therefore \bar{\Delta}_{v-1}=\underline{\min }_{v-1}\left\{\bar{\Delta}_{v-1}^{G 0}, \bar{\Delta}_{v-1}^{G 1}\right\}=-3.287$

$\therefore \Delta_{v-1}=\bar{\Delta}_{v-1}+\bar{C}_{v-1}=3.499$

-Find the next candidate at Step (3):

Group G0

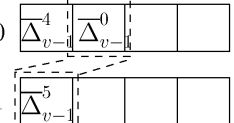

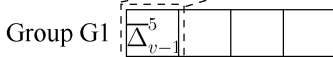

Comparison Window:

$\bar{\Delta}_{v-1}^{G 0}=\bar{\Delta}_{v-1}^{0}=4.364$ (condition $\left|t_{v-1}^{R e 0}\right|>\left|t_{v-1}^{I m 0}\right|$ )

$\bar{\Delta}_{v-1}^{G 1}=\bar{\Delta}_{v-1}^{5}=-0.998$

$\therefore \bar{\Delta}_{v-1}=\min \left\{\bar{\Delta}_{v-1}^{G 0}, \bar{\Delta}_{v-1}^{G 1}\right\}=-0.998$

$\therefore \Delta_{v-1}=\bar{\Delta}_{v-1}+\bar{C}_{v-1}=5.988$

Fig. 4. Example of the soft-decision MSDSD aided D8PSK, which corresponds to the example seen in Fig. 2, subject to the difference that the reduced-complexity algorithms of Tables I and IV are invoked. (a) Example of Reduced-Complexity Soft-Decision MSDSD Conceived for D8PSK Detection. (b) Example of 8PSK constellation diagram visited by ReducedComplexity Soft-Decision SD at index $\mathrm{v}=2$.

in Fig. 2(a) are all leaf nodes. This leads us to the proposed change of the MSDSD output scenario. When the MSDSD algorithm is invoked for the first time, instead of just producing the global optimum $d_{M A P}$ and the MAP hard-bit decisions $\left\{b_{k}^{M A P}\right\}_{k=1}^{\left(N_{w}-1\right) \mathrm{BPS}}$, all the PED values of the leaf nodes $\left\{d_{C A N}=d_{v}\right\}_{\forall v=N_{w}}$ as well as all the corresponding hardbit decisions $\left\{b_{k}^{C A N}\right\}_{k=1}^{\left(N_{w}-1\right) B P S}$ may also be recorded and produced. For example, the SD of Fig. 1(a) may produce both the MAP solution, which is represented by $d_{M A P}=7.28$ and $\left\{b_{k}^{M A P}\right\}_{k=1}^{\left(N_{w}-1\right) \mathrm{BPS}}=1010$, as well as all the leaf nodes, which are represented by $\left\{d_{C A N}\right\}=\{18.4,25.331,7.28$, $16.211\}$ and $\left\{b_{k}^{M A P}\right\}_{k=1}^{\left(N_{w}-1\right) \mathrm{BPS}}=\{1000,1001,1010,1011\}$.

(2) For each soft-bit output, the MSDSD algorithm is invoked again with a fixed bit $b_{k}=\bar{b}_{k}^{M A P}$. Similarly, whenever the SD visits index $v=N_{w}$, the resultant $M$ leaf candidates $\left\{d_{C A N}^{b_{k}=\bar{b}_{k}^{M A P}}\right\}$ may all be recorded and produced. For example, when the SD of Fig. 1(a) is invoked again with a fixed bit $b_{1}=0$, the resultant SD structure is portrayed in Fig. 5, where the two sub-groups of leaf nodes $\{25.118$, $35.09,18.97,14.91\}$ and $\{20.05,24.09,46.615,35.05\}$ may be recorded as $\left\{d_{C A N}^{b_{k}=\bar{b}_{k}^{M A P}}\right\}$. We note that there may only be $M / 2$ leaf candidates, when the fixed bit $b_{k}=\bar{b}_{k}^{M A P}$ is at the specific position in the range of $k \in\left\{\left(N_{w}-\right.\right.$ 2)BPS $\left.+1, \cdots,\left(N_{w}-1\right) \mathrm{BPS}\right\}$.

(3) Finally, the Max-Log-MAP of (20) may be revised for the Approx-Log-MAP as:

$L_{p}\left(b_{k}\right)= \begin{cases}\mathrm{jac}\left(-d_{C A N}\right)-\mathrm{jac}\left(-d_{C A N}^{b_{k}=\bar{b}_{k}^{M A P}}\right), & \text { if } b_{k}^{M A P}=1 \\ \mathrm{jac}\left(-d_{C A N}^{b_{k}=\bar{b}_{k}^{M A P}}\right)-\mathrm{jac}\left(-d_{C A N}\right), & \text { if } b_{k}^{M A P}=0 .\end{cases}$

We note that when the sizes of the two candidate groups $\left\{d_{C A N}\right\}$ and $\left\{d_{C A N}^{b_{k}=\bar{b}_{k}^{M A P}}\right\}$ are not the same, the size of the larger group may be reduced, so that ideally both groups disregard the same number of candidates. Ideally, any potential deviations introduced both by the jac $\left(-d_{C A N}\right)$ and by jac $\left(-d_{C A N}^{b_{k}=\bar{b}_{k}^{M A P}}\right)$ operations may be cancelled out. In practice, the SD's output candidates for $\left\{d_{C A N}\right\}$ and $\left\{d_{C A N}^{b_{k}=\bar{b}_{k}^{M A P}}\right\}$ are always constituted by either the sub-group of $M$ leaf candidates or by the sub-group of $M / 2$ leaf candidates. Therefore, for the larger-sized group, we may compare the best leaf candidates, which are supposed to have the minimum PED values in each sub-groups, and then we may delete the sub-groups associated with the highest locally best leaf candidate's PED value. For the example of Fig. 5, we may delete the sub-group of $\{20.05,24.09,46.615,35.05\}$, because the local best leaf candidates from each sub-group have the relationship of $20.05>14.91$. As a result, the Approx-Log-MAP of (31) may be implemented for the example seen in Figs. 1(a) and 5 as $L_{p}\left(b_{1}\right)=$ jac $(-\{18.4,25.331,7.28,16.211\})-$ jac $(-\{25.118,35.09,18.97,14.91\})$.

One may argue that the SD does not visit all the MSDD candidates, which means that the group sizes of $\left\{d_{C A N}\right\}$ and $\left\{d_{C A N}^{b_{k}=\bar{b}_{k}^{M A P}}\right\}$ seen in (31) may be smaller than the group sizes of $\overline{\mathbf{S}}^{i} \in \overline{\mathbf{S}}_{b_{k}=1}$ and $\overline{\mathbf{S}}^{i} \in \overline{\mathbf{S}}_{b_{k}=0}$ seen in (16). In other words, ideally, the Approx-Log-MAP of (16) may include all the MSDD candidates, but naturally the SD may only visit a subset of them. Nonetheless, as suggested by [39], when the Approx-LogMAP corrects the difference between two probability metrics of $\left|d^{1}-d^{2}\right|$, only 8 values corresponding to $\left|d^{1}-d^{2}\right|$ ranging between 0 and 5.0 may be taken into account. This implies that large differences of $\left|d^{1}-d^{2}\right|>5.0$ are inherently ignored by the Approx-Log-MAP. Therefore, we may assume that the leaf candidates ignored by the SD may also be ignored by the Approx-Log-MAP, so that no extra complexity is imposed on the SD by our proposed Approx-Log-MAP.

We note that for a better implementation, Step (2) may be executed for all $\operatorname{BPS}\left(N_{w}-1\right)$ fixed bits $\left\{b_{k}=\bar{b}_{k}^{M A P}\right\}_{k=1}^{\operatorname{BPS}\left(N_{w}-1\right)}$ before proceeding to Step (3), so that all the leaf nodes visited by the repeated SD searches may be utilized in Step (3). As a result, $\left\{d_{C A N}\right\}$ in (31) may be replaced by $\left\{d_{C A N}^{C_{C}^{C A N}}=b_{k}^{M A P}\right\}$, and then both $\left\{d_{C A N}^{b_{k}^{C A N}=b_{k}^{M A P}}\right\}$ and $\left\{d_{C A N}^{b_{k}=\bar{b}_{k}^{M A P}}\right\}$ in (31) may include all the leaf nodes obtained from Steps (1) and (2) corresponding to the specific bit $b_{k}$ being $b_{k}^{M A P}$ and $\bar{b}_{k}^{M A P}$, respectively. 
TABLE IV

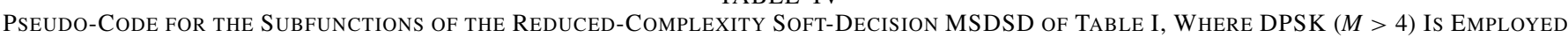

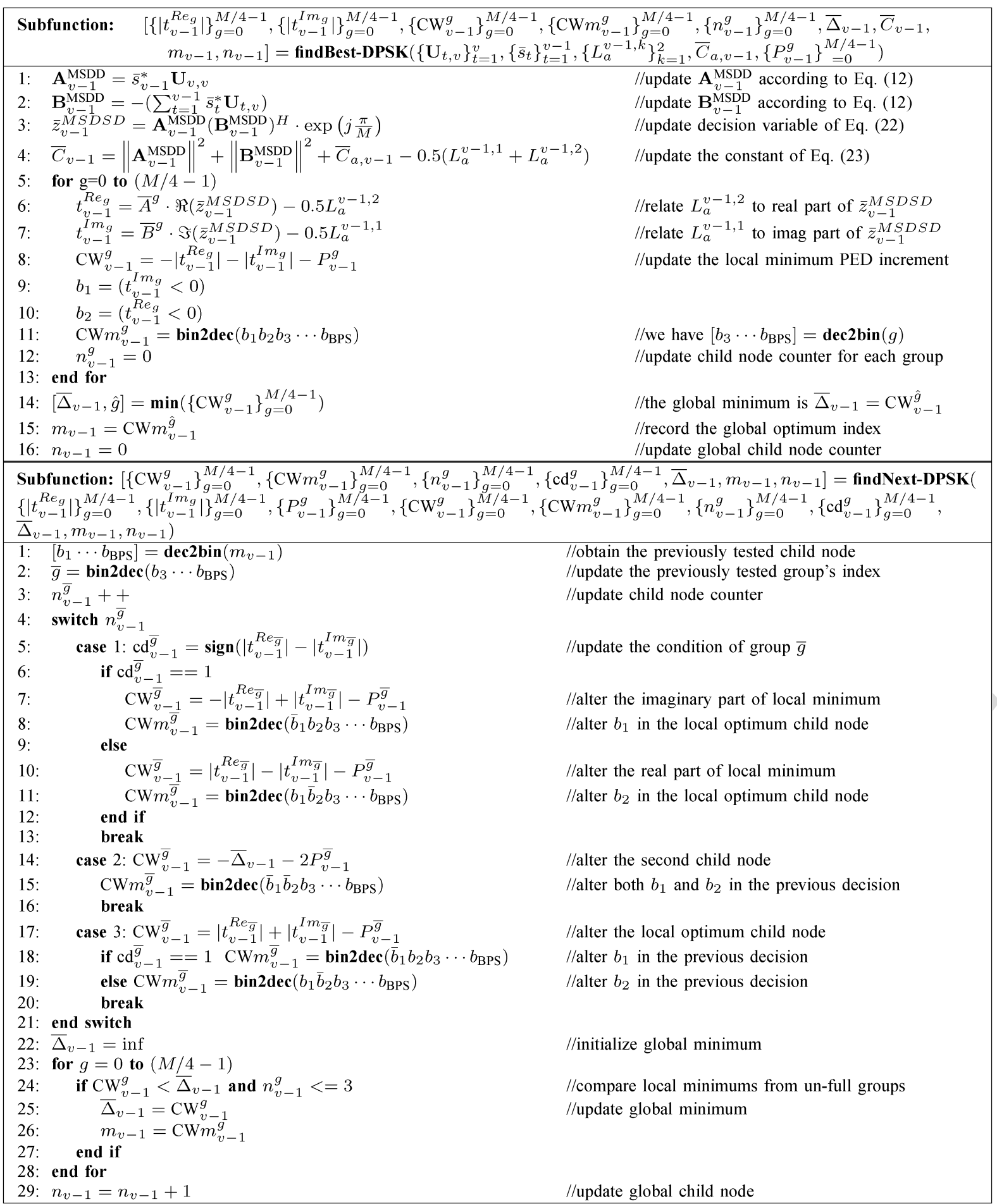

The Approx-Log-MAP may also be straightforwardly applied to the reduced-complexity soft-decision MSDSD of Section IV, where the simplified Schnorr-Euchner strategy of Tables II-IV can be invoked for all SD indice satisfying $v<N_{w}$. However, the original Schnorr-Euchner strategy of [3] has to be invoked for the specific SD index $v=N_{w}$, because all the leaf nodes at $v=N_{w}$ have to be recorded and produced for the Approx-Log-MAP.

\section{Performance Results}

\section{A. Approx-Log-MAP Versus Max-Log-MAP}

First of all, the accuracy of the extrinsic LLRs produced by the Approx-Log-MAP and Max-Log-MAP algorithms are tested as portrayed in Fig. 6, where the two PDFs $\left\{p\left(L_{e} \mid b\right)\right\}_{b=\{0,1\}}$ may be obtained by estimating the histograms of $L_{e}$, with the source bits being $b=\{0,1\}$. If the LLR 


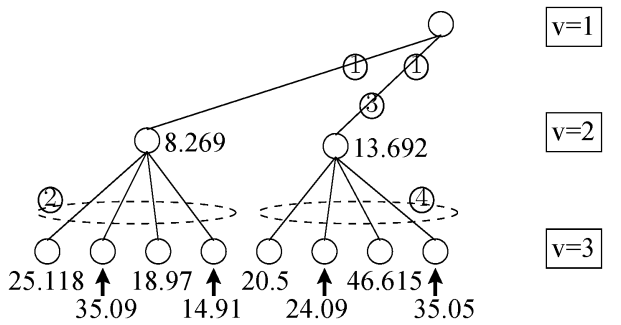

Fig. 5. Example of the soft-decision MSDSD conceived for DQPSK implementing Approx-Log-MAP, which invokes the SD seen in Fig. 1 with a fixed bit of $b_{1}=0$.

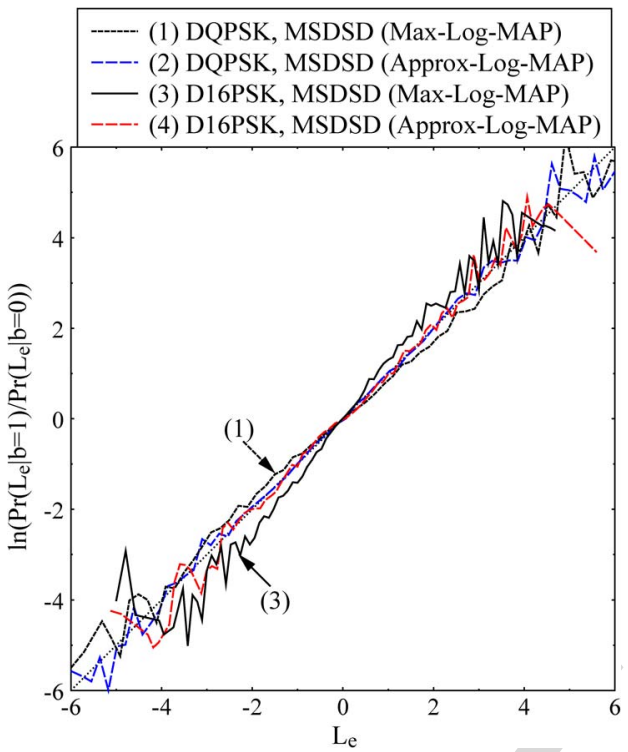

Fig. 6. LLR accuracy test for DQPSK and D16PSK employing both ApproxLog-MAP and Max-Log-MAP aided Subset MSDSD $\left(N_{w}=4\right)$, recorded at $\mathrm{SNR}=0 \mathrm{~dB}$ and $I_{A}=0$, where we have $N_{R}=2$ and $f_{d}=0.03$.

definition of $L_{e}=\ln \frac{p\left(L_{e} \mid b=1\right)}{p\left(L_{e} \mid b=0\right)}$ is statistically true, then the LLRs accuracy test is supposed to result in a diagonal line in Fig. 6. However, the LLRs of the Max-Log-MAP suffer from a noticeable deviation, which is effectively improved by the proposed Approx-Log-MAP, as evidenced by Fig. 6

It is worthy to note that the so-called Subset MSDSD is employed throughout this section. More explicitly, it was discovered in [44] that the symbols at the middle of the MSDSD window may be more reliably detected than those at its edges. Therefore, the Subset MSDSD overlaps the consecutive detection windows by $N_{O L}=3$ observations, so that the $\left(N_{O L}-\right.$ $1=2$ ) symbols detected at the edges may be discarded. ${ }^{5}$ The BER performance of Fig. 7 further confirms the advantage of the proposed Approx-Log-MAP algorithm in both TC coded and IRCC-URC coded DPSK systems, where the simulation parameters are summarized in Table V.

\section{B. Complexity Reduction}

To elaborate, the asymptotic complexity analysis of MSDSD was presented in [45] following the same guideline as the SD

\footnotetext{
${ }^{5}$ We note that the choice of $N_{O L}$ is independent of $N_{w}$, and it was demonstrated in [44] that increasing $N_{O L}$ beyond three does not provide any further advantage.
}

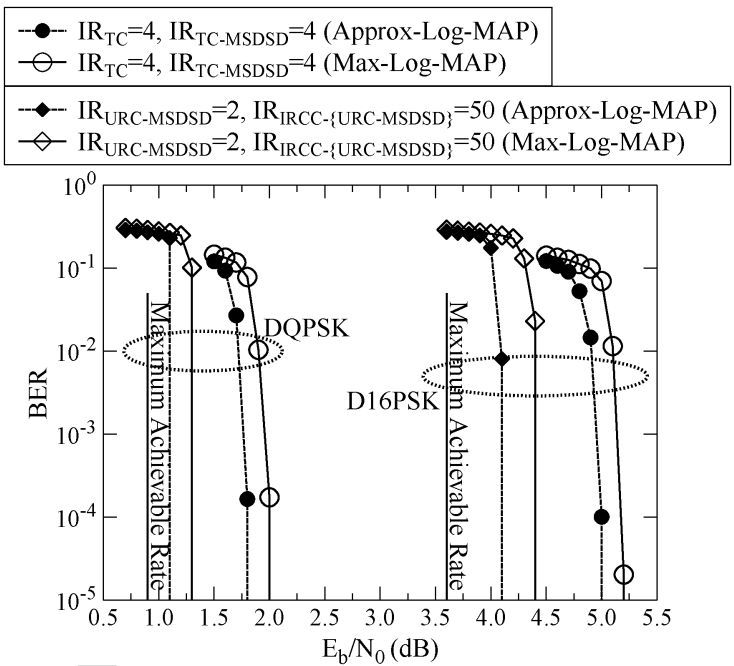

Fig. 7. BER performance of TC/IRCC-URC coded DPSK employing Subset $\operatorname{MSDSD}\left(N_{w}=4\right)$, where we have $N_{R}=2$ and $f_{d}=0.03$.

TABLE V

SYSTEM PARAMETERS

\begin{tabular}{|l|l|}
\hline $\begin{array}{l}\text { Channel } \\
\text { Coding }\end{array}$ & $\begin{array}{l}\text { TC coded DPSK (Schematics Fig. 3.1 in [38]) } \\
\text { IRCC-URC coded DPSK (Schematics Fig. 5.1 in [38]) }\end{array}$ \\
\hline TC coded & $I R_{T C}$ iterations within TC decoder; $I R_{T C}-M S D S D$ \\
DPSK & iterations between TC decoder and MSDSD \\
\hline IRCC-URC & $I R_{U R C-M S D S D}$ iterations between URC decoder and \\
coded & MSDSD; $I_{I R C C-\{U R C-M S D S D\} \text { iterations between }}$ \\
DPSK & IRCC and the amalgamated URC-MSDSD decoder. \\
\hline Frame length & 1000000 bits \\
\hline
\end{tabular}

\begin{tabular}{l|l}
\hline Frame length & 1000000 bits
\end{tabular}

TABLE VI

COMPLEXITY OF SOFT-DECISION MSDSD SUBFUNCTIONS

\begin{tabular}{|c|c|c|c|c|}
\hline & $\begin{array}{l}\text { real-valued mul- } \\
\text { tiplications }\end{array}$ & $\begin{array}{l}\text { real-valued } \\
\text { additions }\end{array}$ & comparisons & $\begin{array}{l}\text { visited } \\
\text { nodes }\end{array}$ \\
\hline sortDelta & $\left(\begin{array}{c}\left(4 N_{R} v+\right. \\
\left.6 N_{R}\right) M\end{array}\right.$ & $\begin{array}{r}\left(4 N_{R} v+\right. \\
\left.2 N_{R}\right) M\end{array}$ & $O(M \log M)$ & $M$ \\
\hline findBest-DBPSK & $4 N_{R} v+8 N_{R}$ & $4 N_{R} v+4 N_{R}$ & 2 & 1 \\
\hline findNext-DBPSK & 0 & 2 & 0 & 1 \\
\hline findBest-DQPSK & $\begin{array}{l}4 N_{R} v+8 N_{R}+ \\
7\end{array}$ & $\begin{array}{l}4 N_{R} v+4 N_{R}+ \\
6\end{array}$ & 4 & 1 \\
\hline findNext-DQPSK & $\leq 1$ & $\leq 4$ & $\leq 3$ & 1 \\
\hline findBest-DPSK & $\begin{array}{l}4 N_{R} v+8 N_{R}+ \\
M+4\end{array}$ & $\begin{array}{c}4 N_{R} v+4 N_{R}+ \\
3 M / 2+2\end{array}$ & $5 M / 4$ & $M / 4$ \\
\hline findNext-DPSK & $\leq 3$ & $\leq 7$ & $\leq M / 2+2$ & 1 \\
\hline
\end{tabular}

aided BLAST of [18], which was only feasible when invoking the sub-optimal Fincke-Pohst strategy of [21]. By contrast, in this work, we focus our attention on the complexity reduction achieved for the optimal Schnorr-Euchner strategy of [1]. Against this background, the detection complexities of the softdecision MSDSD subfunctions are summarized in Table VI, ${ }^{6}$ where "sortDelta" refers to the conventional Schnorr-Euchner search strategy in [3], while the rest of the proposed subfunctions are given by Tables II-IV. It can be seen in Table VI that the proposed Schnorr-Euchner search strategy visits a reduced

\footnotetext{
${ }^{6}$ We note that unneccessary calculations are eliminated for Table VI. For example, both $\left\{0.5 L_{a}^{v-1,1}\right\}_{\forall v}$ and $\left\{0.5 L_{a}^{v-1,2}\right\}_{\forall v}$ may be calculated before invoking the MSDSD's subfunctions. Furthermore, the function of $\left[b_{1} \ldots\right.$ $\left.b_{\mathrm{BPS}}\right]=\operatorname{dec} 2 \mathbf{b i n}(m)$ may be implemented by a pre-stored lookup table for bit-mapping, while its inverse function $m=\operatorname{bin} 2 \operatorname{dec}\left(b_{1} \cdots b_{\mathrm{BPS}}\right)=b_{1}$. $2^{\mathrm{BPS}-1}+b_{2} \cdot 2^{\mathrm{BPS}-2}+\cdots+b_{\mathrm{BPS}-1} \cdot 2+b_{\mathrm{BPS}}$ may require a total number of $(B P S-1)$ multiplications as well as $(B P S-1)$ additions.
} 


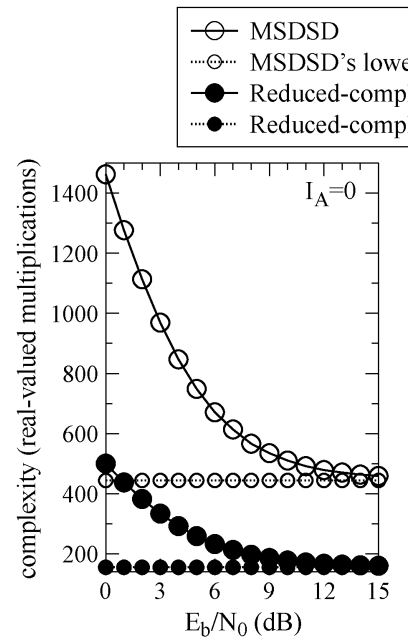

(a)

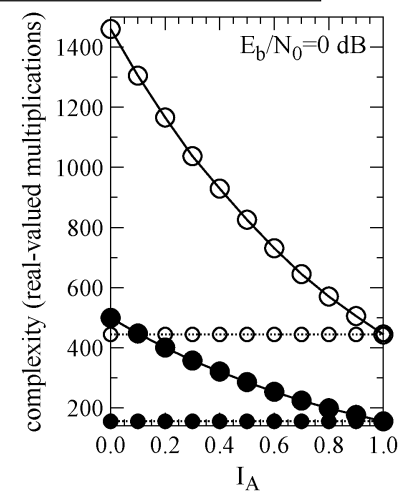

(b)

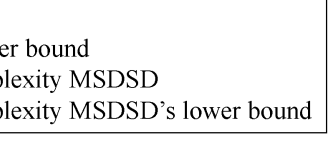

Fig. 8. Complexity (number of real-valued multiplications) comparison between the conventional MSDSD algorithm of [3] associated with $N_{w}=4$ and the reduced-complexity MSDSD algorithm of Tables I and III for coded DQPSK, where we have $N_{R}=2$ and $f_{d}=0.03$. (a) $E_{b} / N_{0}=0 \sim 15 \mathrm{~dB}$. (b) $I_{A}=0 \sim 1$.

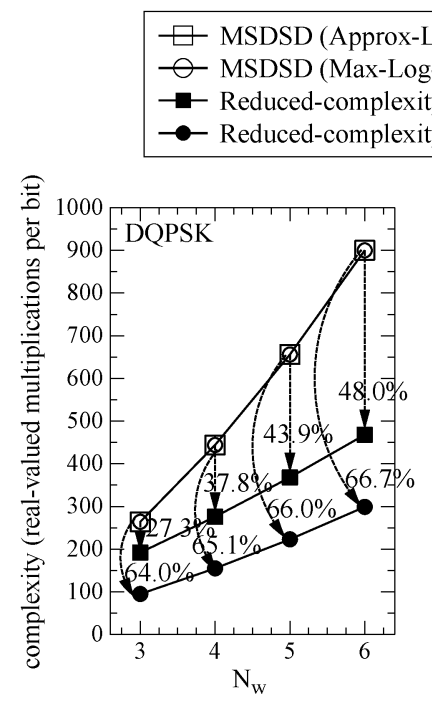

(a)

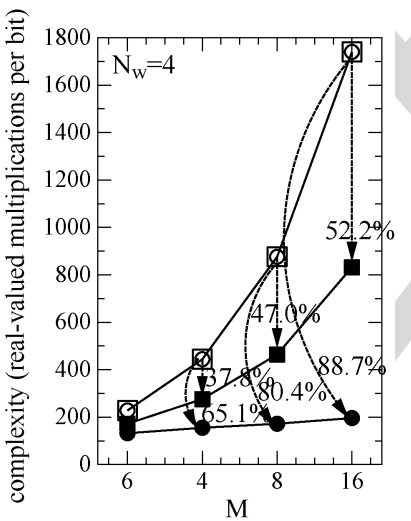

(b)
Fig. 9. Complexity-Reduction Ratio (CRR) achieved by the reducedcomplexity MSDSD algorithm of Table I compared to the conventional MSDSD algorithm of [3] recorded at $E_{b} / N_{0}=0 \mathrm{~dB}$ and $I_{A}=1$, where we have $N_{R}=2$ and $f_{d}=0.03$. (a) $N_{w}=\{3,4,5,6\}$. (b) $M=\{2,4,8,16\}$.

number of nodes, which results in a reduced complexity in all categories.

The complexity of the conventional MSDSD algorithm and that of the proposed MSDSD algorithm conceived for coded DQPSK are compared in Fig. 8 in terms of the total number of real-valued multiplications. It is confirmed by Fig. 8(a) and (b) that the complexities of the MSDSD algorithms may converge to their lower bounds, as $E_{b} / N_{0}$ and/or $I_{A}$ increase. Fig. 8 demonstrates that the proposed MSDSD of Table I substantially reduces the complexity of the conventional MSDSD in [3]. The Complexity-Reduction Ratios (CRRs) achieved by our reduced-complexity design are further presented in Fig. 9.
We note that for the conventional MSDSD, the Approx-LogMAP and the Max-Log-MAP impose the same number of realvalued multiplications. By contrast, for the proposed MSDSD, the Approx-Log-MAP has to invoke the conventional SchnorrEuchner strategy for the specific SD index $v=N_{w}$, which results in a higher number of multiplications than the Max-LogMAP. Nonetheless, Fig. 9(a) and (b) demonstrate that substantial complexity reductions of up to $C R R=48.0 \%$ and $C R R=$ $52.2 \%$ are achieved by the Subset MSDSD $\left(N_{w}=6\right)$ aided DQPSK and by the Subset MSDSD $\left(N_{w}=4\right)$ aided D16PSK, when the Approx-Log-MAP is implemented. Furthermore, even more substantial complexity reductions of $C R R=66.7 \%$ and $C R R=88.7 \%$ are achieved by the Subset $\operatorname{MSDSD}\left(N_{w}=\right.$ 6) aided DQPSK and by the Subset MSDSD $\left(N_{w}=4\right)$ aided D16PSK, when the Max-Log-MAP is implemented. We note that the complexity reductions achieved by the proposed MSDSD are especially significant, when the MSDSD is iteratively invoked several times by the turbo detected systems.

Once again, we note that the proposed soft-decision MSDSD algorithm presented in Tables I-IV has exactly the same detection capability as the conventional soft-decision MSDSD algorithm presented in Appendix I of [3]. We have arranged for them to decode the same channel output associated with the same a priori soft input, and we found that they always produce exactly the same SD decisions.

\section{Coherent Versus Noncoherent}

Last but not least, MSDSD aided DQPSK is compared to its PSAM aided coherent QPSK counterpart. First of all, Fig. 10 ${ }^{7}$ demonstrates that when we have $f_{d}=0.03$, the LLRs produced by the PSAM aided QPSK detector suffer from severe deviation from the true probabilities, which may result in disproportionately high LLR values that may misinform the channel decoder.

Secondly, Fig. $11^{8}$ demonstrates that when we have $f_{d}=0.001$, the coherent PSAM aided QPSK significantly outperforms the Subset MSDSD aided DQPSK in both our TC and IRCC-URC coded systems. However, when the normalized Doppler frequency is increased to $f_{d}=0.03$, the PSAM aided QPSK's performance degrades substantially, while the Subset MSDSD aided DQPSK only suffers from a small BER performance degradation, which gives the noncoherent schemes a $0.7 \mathrm{~dB}$ and a $1.4 \mathrm{~dB}$ performance advantage over their coherent counterparts in the context of our TC and IRCC-URC coded systems, respectively, as evidenced by Fig. 11. Therefore, we may conclude that the DPSK schemes employing MSDSD may be deemed to be a more suitable candidate for channel coded systems operating at high Doppler frequencies.

\footnotetext{
${ }^{7}$ When we have $f_{d}=0.03$, the PSAM's pilot spacing $N_{P S}$ is reduced to 12 in order to sample the channel more frequently, while the PSAM's observation window length $N_{O W}$ (number of filter coefficients) is also reduced to 12 , due to the weak temporal correlation.

${ }^{8}$ There is no iteration between the QPSK detector and the channel decoder in Fig. 11, because the QPSK detection does not produce any iteration gain [28]. Nonetheless, the coded coherent schemes and their noncoherent counterparts have the same total number of iterations for turbo detection.
} 


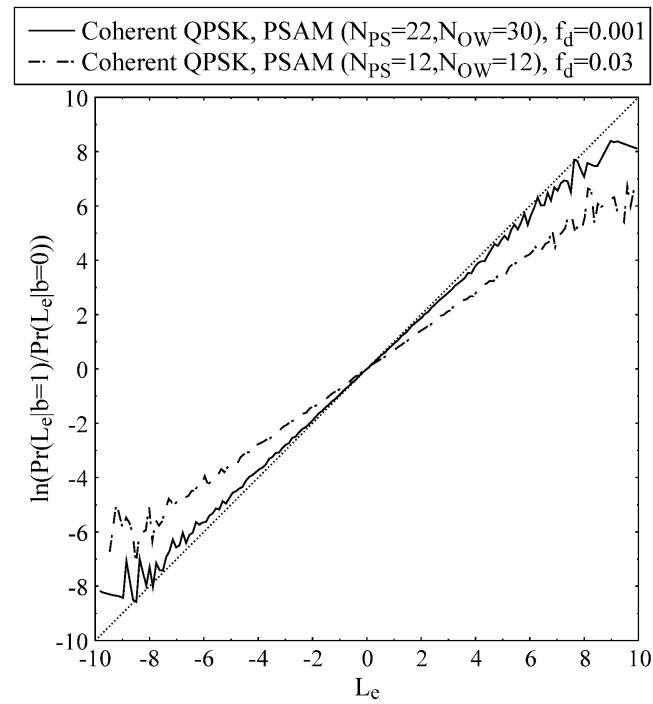

Fig. 10. LLR accuracy test for PSAM [11] aided coherent QPSK, recorded at $\mathrm{SNR}=0 \mathrm{~dB}$ and $I_{A}=0$, where we have $N_{R}=2$.

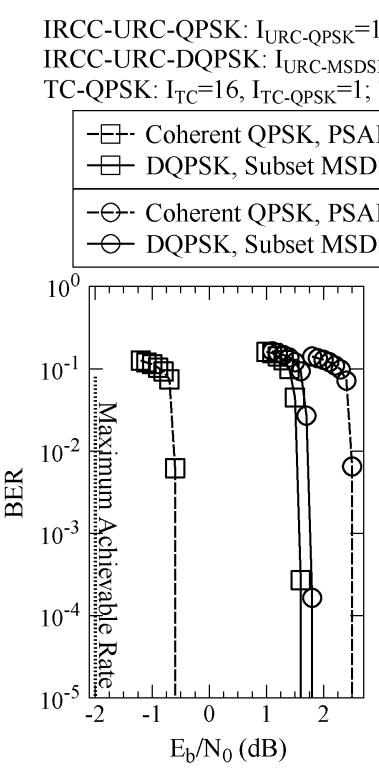

(a)
Fig. 11. BER performance comparison between TC/IRCC-URC coded PSAM [11] aided coherent QPSK and TC/IRCC-URC coded Subset MSDSD aided DQPSK, where the Approx-Log-MAP is invoked, while $N_{R}=2$ receive antennas are used. (a) TC coded systems. (b) IRCC-URC coded systems.

\section{CONCLUSION}

In this paper, the Schnorr-Euchner search strategy was configured to always visit the MPSK constellation points in a zigzag fashion both for the hard-decision MSDSD of Section II and for the soft-decision MSDSD of Section IV. The complexity results of Figs. 8 and 9 demonstrated that our proposed design offers a substantial complexity reduction. Furthermore, the Approx-Log-MAP algorithm implemented by the MSDSD was proposed in Section V, which improved the Max-LogMAP, as demonstrated by Figs. 6 and 7. Finally, our comparison of the channel-coded coherent and noncoherent schemes characterized in Figs. 10 and 11 suggested that the DPSK schemes employing MSDSD may be deemed to be more suitable candidates for channel coded systems operating at high Doppler frequencies.

\section{APPENDIX}

In this section, we aim to conceive the SD aided V-BLAST employing MPSK [29]-[34] in the same form as the MSDSD aided DPSK, so that the reduced-complexity Schnorr-Euchner search strategy proposed in Section IV and the Approx-LogMAP proposed in Section $\mathrm{V}$ may also be applied to the V-BLAST detection.

Explicitly, the $\left(1 \times N_{T}\right)$-element V-BLAST transmission matrix is given by:

$$
\mathbf{S}=\left[s_{1}, \quad \cdots, \quad s_{N_{T}}\right]=\left[\begin{array}{lll}
\frac{s^{m_{1}}}{\sqrt{N_{T}}}, & \cdots, & \frac{s^{m_{N_{T}}}}{\sqrt{N_{T}}}
\end{array}\right],
$$

where the MPSK/QAM symbols are separately modulated as $\left\{s^{m_{v}}\right\}_{v=1}^{N_{T}}$, while the factor $\sqrt{N_{T}}$ normalizes the transmission power. The signal received by the $N_{R}$ antenna elements at the receiver may be modelled as:

$$
\mathbf{Y}=\mathbf{S H}+\mathbf{V},
$$

where the $\left(1 \times N_{T}\right)$-element vector $\mathbf{S}$ and the $\left(1 \times N_{R}\right)$-element vector $\mathbf{Y}$ represent the input and output signals of the MIMO channels, respectively. Furthermore, the $\left(N_{T} \times N_{R}\right)$-element $\mathbf{H}$ matrix of (33) models the MIMO's Rayleigh fading channels, while the $\left(1 \times N_{R}\right)$-element AWGN vector $\mathbf{V}$ of (33) models the zero-mean Gaussian random variables with a common complex variance of $N_{0}$, whose PDF is given by $p(\mathbf{V})=p\left(\mathbf{Y} \mid \mathbf{S}^{i}\right)=$ $\frac{1}{\left(\pi N_{0}\right)^{N_{R} N_{P}}} \exp \left(-\frac{\left\|\mathbf{Y}-\mathbf{S}^{i} \mathbf{H}\right\|^{2}}{N_{0}}\right)$, where there is a total number of $M^{N_{T}}$ combinations $\left\{\mathbf{S}^{i}\right\}_{i=0}^{M^{N_{T}}-1}$ for the MIMO transmission matrix $\mathbf{S}$ in (33). In order to invoke the classic SD, the V-BLAST receiver may apply QR decomposition to $\mathbf{H}^{H}$ [31]-[34], which results in $\mathbf{H}=(\mathbf{Q U})^{H}=\mathbf{L} \mathbf{Q}^{H}$, where the $\left(N_{R} \times N_{T}\right)$-element matrix $\mathbf{Q}$ has orthogonal columns $\mathbf{Q}^{H} \mathbf{Q}=I_{N_{T}}$, while $\mathbf{U}$ and $\mathbf{L}=\mathbf{U}^{H}$ are upper- and lower- triangular matrices, respectively. As a result, (33) may be reformulated as:

$$
\widetilde{\mathbf{Y}}=\mathbf{Y} \mathbf{Q}=\mathbf{S} \mathbf{L}+\mathbf{V Q},
$$

where VQ has exactly the same statistics as the AWGN vector $\mathbf{V}$. Based on the conditional probability $p\left(\mathbf{Y} \mid \mathbf{S}^{i}\right)$ as well as on the Bayes's theorem of (5), the ED to be minimized by the SD may be expressed as [34], [40]:

$$
d=\frac{\sum_{v=1}^{N_{T}}\left|\widetilde{Y}_{v}-\sum_{t=v}^{N_{T}} l_{t, v} s_{t}\right|^{2}}{N_{0}}-\sum_{v=1}^{N_{T}} \sum_{\bar{k}_{v}=1}^{\text {BPS }}\left[\widetilde{b}_{\bar{k}_{v}} L_{a}\left(b_{\bar{k}_{v}}\right)-\bar{C}_{a, \bar{k}_{v}}^{S D}\right],
$$

where an extra constant $\bar{C}_{a, \bar{k}_{v}}^{S D}=\frac{1}{2}\left[\left|L_{a}\left(b_{\bar{k}_{v}}\right)\right|+L_{a}\left(b_{\bar{k}_{v}}\right)\right]$ is introduced in order to guarantee that the ED remains non-negative [34], [40]. According to the ED of (35), the SD's PED may be 
formulated as $d_{v}=d_{v+1}+\Delta_{v}$, where the PED increment $\Delta_{v}$ may be expressed as:

$$
\begin{aligned}
\Delta_{v} & =\frac{\left|\widetilde{Y}_{v}-\sum_{t=v}^{N_{T}} l_{t, v} s_{t}\right|^{2}}{N_{0}}-\sum_{\bar{k}_{v}=1}^{\mathrm{BPS}}\left[\widetilde{b}_{\bar{k}_{v}} L_{a}\left(b_{\bar{k}_{v}}\right)-\bar{C}_{a, \bar{k}_{v}}^{S D}\right] \\
& =\left|\widetilde{A}_{v}^{S D}-s_{v}^{m} \widetilde{B}_{v}^{S D}\right|^{2}-\sum_{\bar{k}_{v}=1}^{\mathrm{BPS}}\left[\widetilde{b}_{\bar{k}_{v}} L_{a}\left(b_{\bar{k}_{v}}\right)-\bar{C}_{a, \bar{k}_{v}}^{S D}\right],
\end{aligned}
$$

where we have $\widetilde{A}_{v}^{S D}=\frac{\widetilde{Y}_{v}-\sum_{t=v+1}^{N_{T}} l_{t, v} s_{t}}{\sqrt{N_{0}}}$ and $\widetilde{B}_{v}^{S D}=\frac{l_{v, v}}{\sqrt{N_{0} N_{T}}}$. As a result, (36) is in the same form as the MSDSD's PED increment of (19) in Section III and (21) in Section IV. Therefore, the reduced-complexity Schnorr-Euchner search strategy and the proposed Approx-Log-MAP solution may be directly applied to the SD aided V-BLAST employing MPSK.

\section{REFERENCES}

[1] C. P. Schnorr and M. Euchner, "Lattice basis reduction: Improved practical algorithms and solving subset sum problems," Math. Programm., vol. 66, no. 1-3, pp. 181-191, Aug. 1994.

[2] L. Lampe, R. Schober, V. Pauli, and C. Windpassinger, "Multiple-symbol differential sphere decoding," IEEE Trans. Commun., vol. 53, no. 12 pp. 1981-1985, Dec. 2005.

[3] V. Pauli, L. Lampe, and R. Schober, "Turbo DPSK' using soft multiplesymbol differential sphere decoding," IEEE Trans. Inf. Theory, vol. 52, no. 4, pp. 1385-1398, Apr. 2006.

[4] J. Lawton, "Theoretical error rates of differentially coherent binary and kineplex data transmission systems," Proc. Inst. Radio Eng., vol. 47, no. 2, pp. 333-334, 1959.

[5] J. Lawton, "Investigation of digital data communication systems," Cornell Aeron. Lab., Buffalo, NY, USA, 1961

[6] C. Cahn, "Performance of digital phase-modulation communication systems," IRE Trans. Commun. Syst., vol. 7, no. 1, pp. 3-6, Jan. 1959.

[7] P. Bello and B. D. Nelin, "The influence of fading spectrum on the binary error probabilites of incoherent and differentially coherent matched filter recievers," IRE Trans. Commun. Syst., vol. 10, no. 2, pp. 160-168, Jun. 1962.

[8] D. Divsalar and M. K. Simon, "Multiple-symbol differential detection of MPSK,” IEEE Trans. Commun., vol. 38, no. 3, pp. 300-308, Mar. 1990.

[9] P. Ho and D. Fung, "Error performance of multiple-symbol differential detection of PSK signals transmitted over correlated Rayleigh fading channels," IEEE Trans. Commun., vol. 40, no. 10, pp. 1566-1569, Oct. 1992.

[10] K. Mackenthun, "A fast algorithm for multiple-symbol differential detection of MPSK," IEEE Trans. Commun., vol. 42, no. 2-4, pp. 1471-1474, Feb.-Apr. 1994.

[11] J. Cavers, "An analysis of pilot symbol assisted modulation for Rayleigh fading channels," IEEE Trans. Veh. Technol., vol. 40, no. 4, pp. 686-693, Nov. 1991.

[12] H. Leib and S. Pasupathy, "The phase of a vector perturbed by Gaussian noise and differentially coherent receivers," IEEE Trans. Inf. Theory, vol. 34, no. 6, pp. 1491-1501, Nov. 1988 .

[13] H. Leib, "Data-aided noncoherent demodulation of DPSK," IEEE Trans. Commun., vol. 43, no. 2-4, pp. 722-725, Feb.-Apr. 1995.

[14] R. Schober, W. Gerstacker, and J. Huber, "Decision-feedback differential detection of MDPSK for flat Rayleigh fading channels," IEEE Trans. Commun., vol. 47, no. 7, pp. 1025-1035, Jul. 1999.

[15] E. Agrell, T. Eriksson, A. Vardy, and K. Zeger, "Closest point search in lattices," IEEE Trans. Inf. Theory, vol. 48, no. 8, pp. 2201-2214, Aug. 2002.

[16] C. Berrou and A. Glavieux, "Near optimum error correcting coding and decoding: Turbo-codes," IEEE Trans. Commun., vol. 44, no. 10, pp. 1261-1271, Oct. 1996.

[17] M. El-Hajjar and L. Hanzo, "EXIT charts for system design and analysis," IEEE Commun. Surveys Tuts., vol. 16, pp. 127-153, 1st Quart. 2014.

[18] J. Jalden and B. Ottersten, "On the complexity of sphere decoding in digital communications," IEEE Trans. Signal Process., vol. 53, no. 4, pp. 1474-1484, Apr. 2005.

[19] B. Hassibi and H. Vikalo, "On the sphere-decoding algorithm I. Expected complexity,” IEEE Trans. Signal Process., vol. 53, no. 8, pp. 2806-2818, Aug. 2005 .
[20] H. Vikalo and B. Hassibi, "On the sphere-decoding algorithm II. Generalizations, second-order statistics, and applications to communications," IEEE Trans. Signal Process., vol. 53, no. 8, pp. 2819-2834, Aug. 2005.

[21] U. Fincke and M. Pohst, "Improved methods for calculating vectors of short length in a lattice, including a complexity analysis," Math. Comput., vol. 44, no. 170 , pp. 463-471, 1985.

[22] A. Kyrillidis and G. N. Karystinos, "Fixed-rank Rayleigh quotient maximization by an MPSK sequence," IEEE Trans. Commun., vol. 62, no. 3 pp. 961-975, Mar. 2014

[23] Z. Pi and F. Khan, "An introduction to millimeter-wave mobile broadband systems,” IEEE Commun. Mag., vol. 49, no. 6, pp. 101-107, Jun. 2011.

[24] T. Rappaport, J. Murdock, and F. Gutierrez, "State of the art in 60-GHz integrated circuits and systems for wireless communications,' Proc. IEEE, vol. 99, no. 8, pp. 1390-1436, Aug. 2011.

[25] T. Marzetta, "Noncooperative cellular wireless with unlimited numbers of base station antennas," IEEE Trans. Wireless Commun., vol. 9, no. 11, pp. 3590-3600, Nov. 2010

[26] F. Rusek et al., "Scaling up MIMO: Opportunities and challenges with very large arrays," IEEE Signal Process. Mag., vol. 30, no. 1, pp. 40-60, Jan. 2013

[27] C. Xu, S. Sugiura, S. X. Ng, and L. Hanzo, "Reduced-complexity noncoherently detected differential space-time shift keying," IEEE Signal Process. Lett., vol. 18, no. 3, pp. 153-156, Mar. 2011.

[28] C. Xu, D. Liang, S. Sugiura, S. X. Ng, and L. Hanzo, "Reduced-complexity approx-log-MAP and max-log-MAP soft PSK/QAM detection algorithms," IEEE Trans. Commun., vol. 61, no. 4, pp. 1415-1425, Apr. 2013.

[29] O. Damen, A. Chkeif, and J. C. Belfiore, "Lattice code decoder for spacetime codes," IEEE Commun. Lett., vol. 4, no. 5, pp. 161-163, May 2000.

[30] M. Damen, H. El-Gamal, and G. Caire, "On maximum-likelihood detection and the search for the closest lattice point," IEEE Trans. Inf. Theory, vol. 49 , no. 10 , pp. $2389-2402$, Oct. 2003

[31] B. Hochwald and S. ten Brink, "Achieving near-capacity on a multipleantenna channel," IEEE Trans. Commun., vol. 51, no. 3, pp. 389-399, Mar. 2003.

[32] H. Vikalo, B. Hassibi, and T. Kailath, "Iterative decoding for MIMO channels via modified sphere decoding," IEEE Trans. Wireless Commun. vol. 3, no. 6, pp. 2299-2311, Jan. 2004.

[33] C. Studer, A. Burg, and H. Bolcskei, "Soft-output sphere decoding: Algorithms and VLSI implementation," IEEE J. Sel. Areas Commun. vol. 26, no. 2, pp. 290-300, Feb. 2008

[34] C. Studer and H. Bolcskei, "Soft-input soft-output single tree-search sphere decoding," IEEE Trans. Inf. Theory, vol. 56, no. 10, pp. 4827 4842, Sep. 2010.

[35] D. Divsalar and M. Simon, "Maximum-likelihood differential detection of uncoded and trellis coded amplitude phase modulation over AWGN and fading channels-Metrics and performance," IEEE Trans. Commun., vol. 42, no. 1, pp. 76-89, Jan. 1994.

[36] R. H. Clarke, "A statistical theory of mobile radio reception," Bell Labs Tech. J., vol. 47, no. 6, pp. 957-1000, Jul./Aug. 1968.

[37] W. Koch and A. Baier, "Optimum and sub-optimum detection of coded data disturbed by time-varying intersymbol interference," in Proc. IEEE GLOBECOM, San Diego, CA, USA, Dec. 1990, vol. 3, pp. 1679-1684

[38] L. Hanzo, O. Alamri, M. El-Hajjar, and N. Wu, Near-Capacity MultiFunctional MIMO Systems: Sphere-Packing, Iterative Detection and Cooperation. Hoboken, NJ, USA: Wiley, May 2009.

[39] P. Robertson, E. Villebrun, and P. Hoeher, "A comparison of optimal and sub-optimal MAP decoding algorithms operating in the log domain," in Proc. IEEE ICC, Seattle, WA, USA, Jun. 1995, vol. 2, pp. 1009-1013

[40] S. Baro, J. Hagenauer, and M. Witzke, "Iterative detection of MIMO transmission using a List-Sequential (LISS) detector," in Proc. IEEE ICC, May 2003, vol. 4, pp. 2653-2657.

[41] J. Jalden and B. Ottersten, "Parallel implementation of a soft output sphere decoder," in Proc. 39th Asilomar Conf. Signals, Syst. Comput., Oct. 2005 pp. 581-585.

[42] T. H. Cormen, C. E. Leiserson, R. L. Rivest, and C. Stein, Introduction to Algorithms. Cambridge, MA, USA: MIT Press, 2001.

[43] M. A. Bender, M. Farach-Colton, and M. Mosteiro, "Insertion sort is O(n $\log$ n)," Theory Comput. Syst., vol. 39, no. 3, pp. 391-397, 2006.

[44] V. Pauli and L. Lampe, "Tree-search multiple-symbol differential decoding for unitary space-time modulation," IEEE Trans. Commun., vol. 55, no. 8, pp. 1567-1576, Aug. 2007.

[45] V. Pauli and L. Lampe, "On the complexity of sphere decoding for differential detection," IEEE Trans. Inf. Theory, vol. 53, no. 4, pp. 1595-1603, Apr. 2007. 


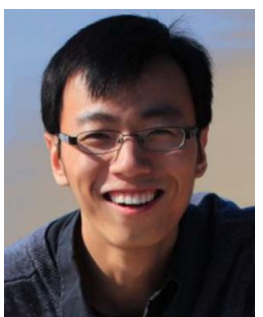

Chao Xu (S'09-M'14) received the B.Eng. degree from Beijing University of Posts and Telecommunications, Beijing, China, and the B.Sc. (Eng.) with First Class Honours from Queen Mary, University of London, London, U.K., through a Sino-U.K. joint degree program in 2008 , both in telecommunications engineering with management. He received the M.Sc. degree with distinction in Radio Frequency Communication Systems and the Ph.D. degree in wireless communications from the University of Southampton, Southampton, U.K., in 2009 and 2015, respectively. He is currently a Postdoctoral Researcher working at Southampton Wireless Group, University of Southampton, Southampton, U.K. His research interests include reduced-complexity multiple-input multiple-output design, noncoherent detection, extrinsic-information-transfer-chart-aided turbo detection, and cooperative communications. He was awarded the Best M.Sc. Student in Broadband and Mobile Communication Networks by the IEEE Communications Society (United Kingdom and Republic of Ireland Chapter) in 2009, and he received 2012 Chinese Government Award for Outstanding Self-Financed Student Abroad.

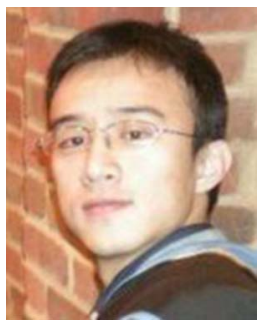

Xin Zuo received the B.Eng. degree in telecommunication engineering from the Beijing University of Posts and Telecommunications (BUPT), Beijing in 2008 , and the M.Sc. degree with distinction in radio frequency communication systems from the University of Southampton, Southampton, U.K., in 2009. $\mathrm{He}$ is currently working towards the Ph.D. degree in the Wireless Communications Group in the University of Southampton. His research interests include the error-tolerant design and the implementation of LDPC decoders.

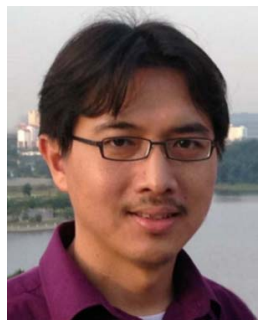

Soon Xin Ng (S'99-M'03-SM'08) received the B.Eng. degree (First Class) in electronic engineering and the Ph.D. degree in telecommunications from the University of Southampton, Southampton, U.K., in 1999 and 2002, respectively. From 2003 to 2006, he was a Postdoctoral Research Fellow working on collaborative European research projects known as SCOUT, NEWCOM, and PHOENIX. Since August 2006, he has been a member of the academic staff in the School of Electronics and Computer Science, University of Southampton. He is involved in the OPTIMIX and CONCERTO European projects as well as the IU-ATC and UC4G projects. He is currently an Associate Professor in telecommunications at the University of Southampton.

His research interests include adaptive coded modulation, coded modulation, channel coding, space-time coding, joint source and channel coding, iterative detection, OFDM, MIMO, cooperative communications, distributed coding, quantum error correction codes and joint wireless-and-optical-fibre communications. He has published over 180 papers and co-authored two John Wiley/IEEE Press books in this field. He is a Chartered Engineer and a Fellow of the Higher Education Academy in the U.K.

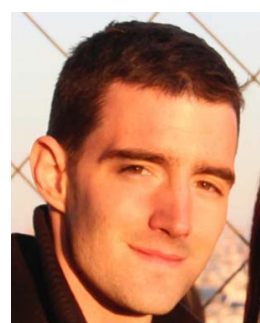

Robert G. Maunder (S'03-M'08-SM'12) received the B.Eng. degree (first class honors) in electronic engineering and the Ph.D. degree in wireless communications from University of Southampton, U.K., in 2003 and 2007, respectively. He became a Lecturer in 2007 and an Associated Professor in 2013. His research interests include joint source/ channel coding, iterative decoding, irregular coding and modulation techniques. For further information on this research, please refer to http://users.ecs.soton. ac.uk/rm

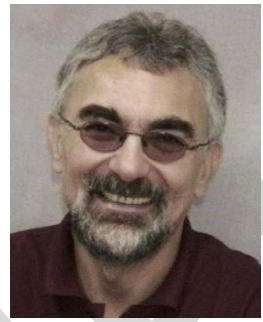

Lajos Hanzo (M'91-SM'92-F'04) received the degree in electronics in 1976 and the doctorate in 1983. In 2009 he was awarded an honorary doctorate by the Technical University of Budapest, while in 2015 by the University of Edinburgh. During his 38-year career in telecommunications he has held various research and academic posts in Hungary, Germany and the U.K. Since 1986, he has been with the School of Electronics and Computer Science, University of Southampton, U.K., where he holds the chair in telecommunications. He has successfully supervised about 100 Ph.D. students, co-authored 20 John Wiley/IEEE Press books on mobile radio communications totalling in excess of 10,000 pages, published $1400+$ research entries at IEEE Xplore, acted both as TPC and General Chair of IEEE conferences, presented keynote lectures, and been awarded a number of distinctions. Currently, he is directing a 60-strong academic research team, working on a range of research projects in the field of wireless multimedia communications sponsored by industry, the Engineering and Physical Sciences Research Council (EPSRC) U.K., the European Research Council's Advanced Fellow Grant and the Royal Society's Wolfson Research Merit Award. He is an enthusiastic supporter of industrial and academic liaison and he offers a range of industrial courses. He is also a Governor of the IEEE Vehicular Technology Society. During 2008-2012, he was the Editor-in-Chief of the IEEE Press and a Chaired Professor also at Tsinghua University, Beijing. His research is funded by the European Research Council's Senior Research Fellow Grant. He has $22000+$ citations. For further information on research in progress and associated publications please refer to http://www-mobile.ecs.soton.ac.uk 


\title{
Reduced-Complexity Soft-Decision Multiple-Symbol Differential Sphere Detection
}

\author{
Chao Xu, Member, IEEE, Xin Zuo, Soon Xin Ng, Senior Member, IEEE, \\ Robert G. Maunder, Senior Member, IEEE, and Lajos Hanzo, Fellow, IEEE
}

\begin{abstract}
Unlike a generic PSK/QAM detector, which may visit a constellation diagram only once, a depth-first Sphere Decoder (SD) has to re-visit the same constellation diagram multiple times. Therefore, in order to prevent the SD from repeating the detection operations, the Schnorr-Euchner search strategy of Schnorr and Euchner may be invoked for optimizing the nodes' search-order, where the ideal case is for the SD to visit the constellation nodes in a zigzag fashion. However, when the hard-decision MultipleSymbol Differential Sphere Detection (MSDSD) of Lampe et al. is invoked for using multiple receive antennas $N_{R} \geq 1$, the SchnorrEuchner search strategy has to visit and sort all the MPSK constellation points. A similar situation is encountered for the softdecision MSDSD of Pauli et al., when the a priori LLRs gleaned from the channel decoder are taken into account. In order to tackle these open problems, in this paper, we propose a correlation process for the hard-decision MSDSD of Lampe $e t$ al. and a reducedcomplexity design for the soft-decision MSDSD of Pauli et al., so that the Schnorr-Euchner search strategy always opts for visiting the MPSK constellation points in a zigzag fashion. Our simulation results demonstrate that a substantial complexity reduction is achieved by our reduced-complexity design without imposing any performance loss. Explicitly, up to $\mathbf{8 8 . 7 \%}$ complexity reduction is attained for MSDSD $\left(N_{w}=4\right)$ aided D16PSK. This complexity reduction is quite substantial, especially when the MSDSD is invoked several times during turbo detection. Furthermore, in order to offer an improved solution and a comprehensive study for the soft-decision MSDSD, we also propose to modify the output of the SD to harmonize its operation with the near-optimum ApproxLog-MAP. Then the important subject of coherent versus noncoherent is discussed in the context of coded systems, which suggests that MSDSD aided DPSK is an eminently suitable candidate for turbo detection assisted coded systems operating at high Doppler frequencies.
\end{abstract}

Index Terms-Multiple-symbol differential sphere detection, DPSK, sphere decoding, Schnorr-Euchner search strategy, turbo detection, soft-decision, coherent versus noncoherent.

Manuscript received December 26, 2014; revised May 5, 2015; accepted July 4, 2015. The financial support of the European Research Council's Advanced Fellow Grant is gratefully acknowledged. The associate editor coordinating the review of this paper and approving it for publication was G. Bauch

The authors are with the School of Electronics and Computer Science, University of Southampton, Southampton SO17 1BJ, U.K. (e-mail: cx1g08@ecs. soton.ac.uk; xz3g08@ecs.soton.ac.uk; sxn@ecs.soton.ac.uk; rm@ecs.soton. ac.uk; 1h@ecs.soton.ac.uk).

Color versions of one or more of the figures in this paper are available online at http://ieeexplore.ieee.org.

Digital Object Identifier 10.1109/TCOMM.2015.2456177

\section{INTRODUCTION}

$\mathbf{T}$ HE history of Differential Phase Shift Keying (DPSK) dates back to Lawton's classic work [4], [5] in 1959-1960, where the effect of false carrier-phase locking is mitigated by the low-complexity Conventional Differential Detection (CDD). More explicitly, the DPSK transmitter modulates the datacarrying symbols onto the phase changes between consecutive transmitted symbols, so that the CDD may recover the source information by observing the phase change between every pair of consecutive received samples. However, it was demonstrated by Cahn [6] in 1959 that the CDD-aided DPSK scheme suffers from a $3 \mathrm{~dB}$ performance penalty compared to its coherent counterpart. Moreover, it was discovered by Bello and Nelin [7] in 1962 that an irreducible error floor occurs for DPSK, when the CDD, which was originally designed for AWGN channels, is directly employed in rapidly fluctuating fading channels. In order to mitigate this problem, the Multiple-Symbol Differential Detection (MSDD) philosophy was proposed by Divsalar and Simon [8] in 1990 for DPSK invoked in AWGN channels and by Ho as well as Fung [9] in 1992 for Rayleigh fading channels. More explicitly, the MSDD extends the CDD's observation window width of $N_{w}=2$ to $N_{w} \geq 2$, where a total number of $\left(N_{w}-1\right)$ data-carrying symbols are jointly detected. The price paid is that the MSDD complexity grows exponentially with $\left(N_{w}-1\right)$. In order to reduce the MSDD complexity, a reduced-complexity algorithm was conceived for MSDD operating in AWGN channels by Mackenthun [10] in 1994, which may also be invoked for slowly-fading channels exhibiting a near-constant envelope for a block of signal transmission. As a closely related result, it was demonstrated by Cavers [11] in 1991 that accurate channel estimation relying on the classic Pilot Symbol Assisted Modulation (PSAM) may become especially challenging, when the normalized Doppler frequency is increased. Therefore, as an attractive alternative to coherent receivers, it is essentially important to implement MSDD in rapidly fluctuating fading channels at an affordable complexity.

Another low-complexity design alternative, namely the Decision-Feedback Differential Detection (DFDD) was originally proposed for AWGN channels by Leib and Pasupathy [12] in 1988. Then in 1995 it was confirmed by Leib [13] that the DFDD of [12] is equivalent to the MSDD of [8] operating in decision-feedback mode. The DFDD design was further extended to Rayleigh fading channels by Schober et al. [14] in 1999. However, the DFDD inevitably imposes a performance loss due to its inherent error-propagation problem. In order to retain the optimum MSDD performance, the state-of-the-art 
Multiple-Symbol Differential Sphere Detection (MSDSD) was proposed by Lampe et al. [2] in 2005, where the MSDD is facilitated by invoking the Sphere Decoder (SD) [15]. Furthermore, inspired by the revolutionary Turbo Code (TC) concept [16] and the generalization of the "turbo principle" [17], the softdecision MSDSD was conceived for DPSK by Pauli et al. [3] in 2006, so that the MSDSD may be invoked in turbo detection for the sake of approaching the full capacity potential of the DPSK systems.

Although the MSDD complexity may be effectively reduced by the SD, it was demonstrated by Jalden and Ottersten [18] in 2005 that the SD complexity still remains an exponential function at low SNRs. Later, Hassibi and Vikalo [19], [20] demonstrated that the expected SD complexity invoking the Fincke-Pohst enumeration strategy [21] obeyed a polynomial function. As an alternative, Kyrillidis and Karystinos [22] recently proposed a new algorithm that aimed for maximizing the Rayleigh quotient of PSK sequence detection in 2014, where the complexity was a fixed polynomial function at all SNRs. Nonetheless, further research efforts are required for enhancing this algorithm [22] in soft-decision-aided turbo detection assisted coded systems. Therefore, in this paper, we focus our attention on the MSDSD solutions [2], [3].

The classic MSDSD aided DPSK [2] was first invoked for a single receive antenna $\left(N_{R}=1\right)$. Since the recent developments in the millimeter-wave band [23], [24] facilitate the employment of a large number of antennas, especially at the Base Station (BS) [25], [26], DPSK systems relying on multiple receive antennas $N_{R} \geq 1$ may be preferred. However, when the harddecision MSDSD of [2] is invoked for $N_{R} \geq 1$, the SchnorrEuchner search strategy has to visit and sort all the MPSK constellation points. The similar situation is encountered for the soft-decision MSDSD of [3], when the a priori LLRs gleaned from the channel decoder are taken into account. Against this background, we offer the following novel contributions in this paper:

1) We propose to introduce a low-complexity correlation operation into the hard-decision MSDSD aided DPSK scheme employing an arbitrary number of receive antennas, so that the SD may visit the constellation points in a zigzag fashion, which is similar to the scenario of $N_{R}=1$ in [2].

2) It was shown in [27], [28] that substantial complexity reduction may be attained by exploring the symmetry of the Gray-labelled MPSK constellation. Against this background, we propose a reduced-complexity SchnorrEuchner search strategy for the soft-decision MSDSD of [3] employing an arbitrary number of received antennas. The proposed soft-decision MSDSD algorithm may visit a reduced number of nodes and hence achieve a substantial complexity reduction without imposing any performance loss.

Moreover, in order to offer an improved solution and a comprehensive study for the soft-decision MSDSD, the following novelties are also offered in this paper:

3) The soft-decision MSDSD proposed in [3] invokes the suboptimal Max-Log-MAP algorithm, where the SD produces only the optimum candidate. Against this background, we additionally propose to modify the output of the SD, where multiple candidates may be produced so that the nearoptimum Approx-Log-MAP may be implemented.

4) Furthermore, the important subject of coherent versus noncoherent detection is discussed for coded systems. Our simulation results suggest that compared to the coherent MPSK relying on realistic channel estimation, the DPSK schemes employing MSDSD may be deemed as a more suitable candidate for turbo detection aided coded systems operating at high Doppler frequencies.

The rest of this paper is organized as follows. The harddecision MSDSD of [2] is extended to the case of $N_{R} \geq 1$ in Section II, where the correlation operation is introduced. The soft-decision MSDSD of [3] is introduced in Section III, and then our reduced-complexity soft-decision MSDSD is proposed in Section IV. Furthermore, the near-optimum Approx-LogMAP for the soft-decision MSDSD is proposed in Section V. We provide simulation results in Section VI, where the coherent versus noncoherent discussion is offered, and our conclusions are given in Section VII. Finally, in Appendix, we present the classic SD aided V-BLAST employing MPSK [29]-[34] in the same form as the MSDSD aided DPSK, so that the proposed reducedcomplexity Schnorr-Euchner search strategy and the ApproxLog-MAP solution may be applied to the V-BLAST detection.

The following notations are used throughout the paper. The operations $(\cdot)^{*}$ and $(\cdot)^{H}$ denote the conjugate of a complex number and the Hermitian transpose of a complex matrix, respectively. The notations $\ln (\cdot)$ and $\exp (\cdot)$ refer to natural logarithm and natural exponential functions, respectively. The notations $p(\cdot)$ and $\mathrm{E}(\cdot)$ denote the probability and the expectation, respectively. The operation $\otimes$ represents the Kronecker product. The notation $\operatorname{rvec}(\mathbf{A})$ forms a row vector by taking the rows of matrix A one-by-one, while Toeplitz(a) refers to the symmetric Toeplitz matrix generated from the vector a. Moreover, the notations $\Re(\cdot)$ and $\Im(\cdot)$ take the real part and the imaginary part of a complex number, respectively.

\section{HARD-DECISION MSDSD}

For an $M$-ary DPSK scheme, the transmitter firstly maps BPS $=\log _{2} M$ source bits $\left\{b_{k}\right\}_{k=1}^{\text {BPS }}$ to an MPSK symbol $x^{m}=$ $\exp \left(j \frac{2 \pi}{M} \check{m}\right)$, where the phasor index $m=\operatorname{bin} 2 \operatorname{dec}\left(b_{1} \cdots b_{\mathrm{BPS}}\right)$ is the Gray-coded index $\check{m}$. Following this, the differential encoding may be performed as:

$$
s_{n}=x_{n-1} s_{n-1} .
$$

For a Single-Input Multiple-Output (SIMO) system, the signal received by the $N_{R}$ receive antennas may be expressed as:

$$
\mathbf{Y}_{n}=s_{n} \mathbf{H}_{n}+\mathbf{V}_{n},
$$

where the $\left(1 \times N_{R}\right)$-element vectors $\mathbf{Y}_{n}, \mathbf{H}_{n}$ and $\mathbf{V}_{n}$ refer to the received signal vector, the Rayleigh fading vector and the AWGN vector which has a zero mean and a variance of $N_{0}$, respectively. Furthermore, the $N_{w}$ observations of (2) may be modelled by MSDD as [9], [35]:

$$
\mathbf{Y}=\mathbf{S H}+\mathbf{V},
$$


where we drop the time index $n$, so that the matrices in (3) are given by $\mathbf{Y}=\left[\mathbf{Y}_{N_{w}}^{T}, \cdots, \mathbf{Y}_{1}^{T}\right]^{T}, \mathbf{S}=\operatorname{diag}\left\{\left[s_{N_{w}}, \cdots, s_{1}\right]\right\}, \mathbf{H}=$ $\left[\mathbf{H}_{N_{w}}^{T}, \cdots, \mathbf{H}_{1}^{T}\right]^{T}$ and $\mathbf{V}=\left[\mathbf{V}_{N_{w}}^{T}, \cdots, \mathbf{V}_{1}^{T}\right]^{T}$. We note that the matrices $\mathbf{Y}, \mathbf{H}$ and $\mathbf{V}$ are of size $\left(N_{w} \times N_{R}\right)$, while $\mathbf{S}$ has $\left(N_{w} \times N_{w}\right)$ elements. Furthermore, since the first transmitted symbol $s_{1}$ in $\mathbf{S}$ is a common phase rotation of the following symbols $\left\{s_{t}\right\}_{t=2}^{N_{w}}$, the MSDD's received signal model of (3) may be rewritten as ${ }^{1}$ :

$$
\mathbf{Y}=\overline{\mathbf{S}} \overline{\mathbf{H}}+\mathbf{V}
$$

where the $v^{\text {th }}$ diagonal element in $\overline{\mathbf{S}}$ is given by $\bar{s}_{v}=s_{v} \cdot s_{1}^{*}$, which leads to $\bar{s}_{1}=1$ and $\bar{s}_{v}=x_{v-1} \bar{s}_{v-1}=\prod_{t=1}^{v-1} x_{t}$ for $v>1$, while the $v^{\text {th }}$ row in $\overline{\mathbf{H}}$ is given by $\overline{\mathbf{H}}_{v}=s_{1} \mathbf{H}_{v}$. The MSDD aims for maximizing the following a posteriori probability:

$$
p\left(\overline{\mathbf{S}}^{i} \mid \mathbf{Y}\right)=\frac{p\left(\mathbf{Y} \mid \overline{\mathbf{S}}^{i}\right) p\left(\overline{\mathbf{S}}^{i}\right)}{\sum_{\forall \overline{\mathbf{S}}} p\left(\mathbf{Y} \mid \overline{\mathbf{S}}^{j}\right) p\left(\overline{\mathbf{S}} \bar{j}^{j}\right.},
$$

where the a priori probability $p\left(\overline{\mathbf{S}}^{i}\right)$ may be assumed to be a constant of $\frac{1}{M^{\left(N_{W}-1\right)}}$ for the equiprobable candidates $\left\{\overline{\mathbf{S}}_{\}_{i=0}^{i}}^{M^{\left(N_{w}-1\right)}-1}\right.$. Furthermore, the conditional probability $p\left(\mathbf{Y} \mid \overline{\mathbf{S}}^{i}\right)$ in (5) may be expressed as:

$$
p\left(\mathbf{Y} \mid \overline{\mathbf{S}}^{i}\right)=\frac{\exp \left\{-\operatorname{rvec}(\mathbf{Y}) \cdot \mathbf{R}_{Y Y}^{-1} \cdot[\operatorname{rvec}(\mathbf{Y})]^{H}\right\}}{\pi^{N_{R} N_{w}} \operatorname{det}\left(\mathbf{R}_{Y Y}\right)},
$$

where the equivalent signal model is given by $\operatorname{rvec}(\mathbf{Y})=$ $\operatorname{rvec}(\overline{\mathbf{H}}) \cdot\left(\overline{\mathbf{S}} \otimes \mathbf{I}_{N_{R}}\right)+\operatorname{rvec}(\mathbf{V})$. As a result, the correlation matrix in (6) may be formulated as:

$$
\mathbf{R}_{Y Y}=\mathrm{E}\left\{[\operatorname{rvec}(\mathbf{Y})]^{H} \cdot \operatorname{rvec}(\mathbf{Y})\right\}=\left[\left(\overline{\mathbf{S}}^{i}\right)^{H} \mathbf{C} \overline{\mathbf{S}}^{i}\right] \otimes \mathbf{I}_{N_{R}} .
$$

More explicitly, the fading correlation matrix is given by $\mathbf{R}_{\bar{H} \bar{H}}=\mathrm{E}\left\{[\operatorname{rvec}(\overline{\mathbf{H}})]^{H} \cdot \operatorname{rvec}(\overline{\mathbf{H}})\right\}=\mathbf{R}_{h h} \otimes \mathbf{I}_{N_{R}}$, where the fading characteristic matrix is given by $\mathbf{R}_{h h}=\operatorname{Toeplitz}\left(\left[\rho_{0}, \rho_{1}, \cdots\right.\right.$, $\left.\rho_{N_{w}-1}\right]$ ). According to the Clarke model [36], the fading autocorrelation is given by $\left\{\rho_{v}=J_{0}\left(2 \pi f_{d} \cdot v\right)\right\}_{v=0}^{N_{w}-1}$, where $J_{0}(\cdot)$ is the zero-order Bessel function of the first kind, while $f_{d}$ denotes the normalized Doppler frequency. Moreover, the AWGN correlation matrix is given by $\mathbf{R}_{V V}=\mathrm{E}\left\{[\operatorname{rvec}(\mathbf{V})]^{H} \cdot \operatorname{rvec}(\mathbf{V})\right\}=$ $\mathbf{R}_{v v} \otimes \mathbf{I}_{N_{R}}$, where the AWGN characteristic matrix is simply given by $\mathbf{R}_{v v}=N_{0} \mathbf{I}_{N_{w}}$. As a result, the channel characteristic matrix in (7) is given by $\mathbf{C}=\mathbf{R}_{h h}+\mathbf{R}_{v v}$. The transmission matrix $\overline{\mathbf{S}}$ of (3) is a unitary matrix, hence the determinant term in (6) is a constant of $\operatorname{det}\left(\mathbf{R}_{Y Y}\right)=\left\{\operatorname{det}\left[\left(\overline{\mathbf{S}}^{i}\right)^{H} \cdot \operatorname{det}(\mathbf{C})\right.\right.$. $\left.\left.\operatorname{det}\left(\overline{\mathbf{S}}^{i}\right)\right]\right\}^{N_{R}}=\operatorname{det}(\mathbf{C})^{N_{R}}$. In summary, the MSDD that maximizes the a posteriori probability of (5) may be formulated as:

$$
\hat{\mathbf{S}}=\arg \min _{\forall \overline{\mathbf{S}} i}\left\|\mathbf{L}\left(\overline{\mathbf{S}}^{i}\right)^{H} \mathbf{Y}\right\|^{2},
$$

where $\mathbf{L}$ is a lower triangular matrix obtained from the Cholesky decomposition of $\mathbf{C}^{-1}=\mathbf{L L}^{H}$.

\footnotetext{
${ }^{1}$ We note that $\mathbf{Y}=\left[\mathbf{Y}_{N_{w}}^{T}, \cdots, \mathbf{Y}_{1}^{T}\right]^{T}$ in (3) stores the received signal vectors in a reverse order compared to $\mathbf{Y}=\left[\mathbf{Y}_{1}^{T}, \cdots, \mathbf{Y}_{N_{w}}^{T}\right]^{T}$ seen in [2], [3], so that the MSDSD may detect the transmitted symbols according to their differential encoding order of $\bar{s}_{v}=x_{v-1} \bar{s}_{v-1}$, instead of detecting them backwards as $\bar{s}_{v}=x_{v}^{*} \bar{s}_{v+1}$.
}

In order to facilitate SD ${ }^{2}$ the MSDD metric of (8) may be revised to the Euclidean Distance (ED) as:

$$
\left\|\mathbf{L}^{H} \overline{\mathbf{S}}^{H} \mathbf{Y}\right\|^{2}=\sum_{v=1}^{N_{w}}\left\|\sum_{t=1}^{v} \bar{s}_{t}^{*} \mathbf{U}_{t, v}\right\|^{2} \leq R^{2},
$$

where $R$ denotes the SD's sphere radius. The vectors $\left\{\left\{\mathbf{U}_{t, v}=\right.\right.$ $\left.\left.l_{N_{w}-t+1, N_{w}-v+1} \mathbf{Y}_{t}\right\}_{t=1}^{v}\right\}_{v=1}^{N_{w}}$ in (9) are invariant over the variables $\left\{\bar{s}_{v}\right\}_{v=2}^{N_{w}}$, where $l_{N_{w}-t+1, N_{w}-v+1}$ refers to the element taken from the $\left(N_{w}-t+1\right)$-th row and $\left(N_{w}-v+1\right)$-th column of the lower triangular matrix $\mathbf{L}$ in (8). We note that the superscript $i$ for $\overline{\mathbf{S}}^{i}$ in (8) is omitted in (9) for notational convenience. Therefore, the MSDSD's Partial Euclidean Distance (PED) based on the ED of (9) may be defined as [2]:

$$
d_{v}=\sum_{\bar{v}=1}^{v}\left\|\sum_{t=1}^{\bar{v}} \bar{s}_{t}^{*} \mathbf{U}_{t, \bar{v}}\right\|^{2}=d_{v-1}+\Delta_{v-1},
$$

and the associated PED increment as:

$$
\Delta_{v-1}=\left\|\sum_{t=1}^{v} \bar{s}_{t}^{*} \mathbf{U}_{t, v}\right\|^{2}=\left\|\bar{s}_{v-1}^{*} \mathbf{U}_{v, v}+x_{v-1}\left(\sum_{t=1}^{v-1} \bar{s}_{t}^{*} \mathbf{U}_{t, v}\right)\right\|^{2} .
$$

Observe in (10) and (11) that for a specific index $v$, all the previously tested transmitted symbols $\left\{\bar{s}_{t}\right\}_{t=1}^{v-1}$ have been decided, and the current SD search may opt for the best candidate for representing $x_{v-1}$, which is supposed to minimize $\Delta_{v-1}$.

When $N_{R}=1$ is used, it was demonstrated in [2] that the best phasor index is given by $\breve{m}_{v-1}=\left\lfloor p_{v-1}\right\rceil$, where we have $p_{v-1}=\frac{M}{2 \pi} L\left(-\bar{s}_{v-1}^{*} \mathbf{U}_{v, v} / \sum_{t=1}^{v-1} \bar{s}_{t}^{*} \mathbf{U}_{t, v}\right)$. If the phase index $\check{m}_{v-1}$ was rounded down from $p_{v-1}$, i.e. we have the condition of $p_{v-1}-\breve{m}_{v-1} \geq 0$, then the SD visits the remaining phasors in a zigzag fashion according to the steps of $\breve{m}_{v-1}=\breve{m}_{v-1}+1$, $\breve{m}_{v-1}=\breve{m}_{v-1}-2, \breve{m}_{v-1}=\check{m}_{v-1}+3$, etc. Otherwise, the SD may visit the remaining phasors according to the steps of $\check{m}_{v-1}=$ $\check{m}_{v-1}-1, \check{m}_{v-1}=\check{m}_{v-1}+2, \check{m}_{v-1}=\check{m}_{v-1}-3$, etc. However, for the more general case of using $N_{R}>1,\left\{\left\{\mathbf{U}_{t, v}\right\}_{t=1}^{v}\right\}_{v=1}^{N_{w}}$ in (11) become vectors, hence we cannot directly obtain $p_{v-1}$. In order to mitigate this problem, we rewrite (11) as:

$$
\Delta_{v-1}=\left\|\mathbf{A}_{v-1}^{\mathrm{MSDD}}-x_{v-1} \mathbf{B}_{v-1}^{\mathrm{MSDD}}\right\|^{2}
$$

where we have $\mathbf{A}_{v-1}^{\mathrm{MSDD}}=\bar{s}_{v-1}^{*} \mathbf{U}_{v, v}$ and $\mathbf{B}_{v-1}^{\mathrm{MSDD}}=-\sum_{t=1}^{v-1} \bar{s}_{t}^{*} \mathbf{U}_{t, v}$. As a result, a simple correlation process leads us to the following decision variable:

$$
z_{v-1}=\mathbf{A}_{v-1}^{\mathrm{MSDD}}\left(\mathbf{B}_{v-1}^{\mathrm{MSDD}}\right)^{H},
$$

which may be directly used for detecting $x_{v-1}$. More explicitly, the best phasor is now given by $\check{m}_{v-1}=\left\lfloor p_{v-1}\right\rceil$, where we have $p_{v-1}=\frac{M}{2 \pi} \angle z_{v-1}$, and the Schnorr-Euchner search strategy may visit the remaining phasors in a zigzag fashion in the same way as the case of $N_{R}=1$ in [2].

\footnotetext{
${ }^{2}$ We note that the depth-first tree search strategy of [30] and the SchnorrEuchner constellation search strategy of [1] constitute the default choice for the MSDSD [2], [3], which enables the MSDSD to achieve the same detection capability as the MSDD.
} 


\section{SOFT-DECISION MSDSD}

According to the a posteriori probability of (5), the optimum Log-MAP algorithm invoked by MSDD may be expressed as [37], [38]

$$
\begin{aligned}
L_{p}\left(b_{k}\right) & =\ln \frac{\sum_{\overline{\mathbf{S}}^{i} \in \overline{\mathbf{S}}_{b_{k}=1}} p\left(\overline{\mathbf{S}}^{i} \mid \mathbf{Y}\right)}{\sum_{\overline{\mathbf{S}}^{i} \in \overline{\mathbf{S}}_{b_{k}=0}} p\left(\overline{\mathbf{S}}^{i} \mid \mathbf{Y}\right)} \\
& =\ln \frac{\sum_{\overline{\mathbf{S}}^{i} \in \overline{\mathbf{S}}_{b_{k}=1}} \exp \left(d^{i}\right)}{\sum_{\overline{\mathbf{S}}^{i} \in \overline{\mathbf{S}}_{b_{k}=0}} \exp \left(d^{i}\right)}=L_{a}\left(b_{k}\right)+L_{e}\left(b_{k}\right),
\end{aligned}
$$

where $L_{p}\left(b_{k}\right), L_{e}\left(b_{k}\right)$ and $L_{a}\left(b_{k}\right)$ represent the a posteriori LLR and the extrinsic LLR produced by the MSDD as well as the a priori LLR gleaned from a channel decoder, respectively. Furthermore, $\overline{\mathbf{S}}_{b_{k}=1}$ and $\overline{\mathbf{S}}_{b_{k}=0}$ refer to the MSDD signal set $\overline{\mathbf{S}}$, when the specific bit $b_{k}$ is set to 1 and 0 , respectively. The probability metric ${ }^{3}\left\{d^{i}\right\}_{i=0}^{M^{\left(N_{w}-1\right)}-1}$ seen in (14) is given by $d^{i}=$ $-\left\|\mathbf{L}^{H}\left(\overline{\mathbf{S}}^{i}\right)^{H} \mathbf{Y}\right\|^{2}+\sum_{\bar{k}=1}^{\left(N_{w}-1\right) \mathrm{BPS}} \widetilde{b}_{\bar{k}} L_{a}\left(b_{\bar{k}}\right)$, where $\left\{\widetilde{b}_{\bar{k}}\right\}_{\bar{k}=1}^{\left(N_{w}-1\right) \mathrm{BPS}}$ denotes the bit-mapping corresponding to the MSDD candidate $\mathbf{S}^{i}$. The Log-MAP algorithm of (14) may be simplied by the low-complexity Max-Log-MAP [37] as:

$$
L_{p}\left(b_{k}\right)=\max _{\overline{\mathbf{S}}^{i} \in \overline{\mathbf{S}}_{b_{k}=1}} d^{i}-\max _{\overline{\mathbf{S}}^{i} \in \overline{\mathbf{S}}_{b_{k}=0}} d^{i} .
$$

Furthermore, in order to compensate for the sub-optimum MaxLog-MAP, the Approx-Log-MAP was introduced as [39]:

$$
L_{p}\left(b_{k}\right)=\operatorname{jac}_{\overline{\mathbf{S}}^{i} \in \overline{\mathbf{S}}_{b_{k}=1}} d^{i}-\operatorname{jac}_{\overline{\mathbf{S}}^{i} \in \overline{\mathbf{S}}_{b_{k}}=0} d^{i} .
$$

where jac denotes the Jacobian algorithm of $\operatorname{jac}\left(d^{1}, d^{2}\right)=$ $\max \left\{d^{1}, d^{2}\right\}+\Gamma\left\{\left|d^{1}-d^{2}\right|\right\}$, while the additional term of $\Gamma\left\{\left|d^{1}-d^{2}\right|\right\}$ may take into account the difference between $d^{1}$ and $d^{2}$ according to a lookup table.

The Max-Log-MAP aims for finding the maximum probability metrics, which is similar to the action of hard-decision detectors. Therefore, in order to invoke SD for the Max-LogMAP, the maximization of (15) has to be revised for the sake of minimization, while the probability metrics should be guaranteed to have positive values. As a result, the MSDD probability metric of (14) may be transformed into:

$$
d=\sum_{v=1}^{N_{w}}\left\|\sum_{t=1}^{v} \bar{s}_{t}^{*} \mathbf{U}_{t, v}\right\|^{2}-\sum_{v=2}^{N_{w}} \sum_{\bar{k}_{v}=1}^{\text {BPS }}\left[\tilde{b}_{\bar{k}_{v}} L_{a}\left(b_{\bar{k}_{v}}\right)-\bar{C}_{a, \bar{k}_{v}}\right],
$$

where the superscript $i$ for $d^{i}$ seen in (14) is deleted for the sake of convenience, while the polarity of $d^{i}$ in (14) is altered. Furthermore, the constant $\bar{C}_{a, \bar{k}_{v}}$ in (17) was formulated as $\bar{C}_{a, \bar{k}_{v}}=$ $\ln \prod_{k_{v}=1}^{\mathrm{BPS}}\left\{1+\exp \left[L_{a}\left(b_{\bar{k}_{v}}\right)\right]\right\}$ in [3], which was originally

\footnotetext{
${ }^{3}$ We note that all multiplicative factors of the $\exp \left(d^{i}\right)$ term are eliminated by the division operation in (14), which include the denominator $\left[\sum_{\forall \overline{\mathbf{S}}^{j}} p\left(\mathbf{Y} \mid \overline{\mathbf{S}}^{j}\right) p\left(\overline{\mathbf{S}}^{j}\right)\right]$ of $p\left(\overline{\mathbf{S}}^{i} \mid \mathbf{Y}\right)$ in (5), the denominator $\left[\pi^{N_{R} N_{w}} \operatorname{det}\left(\mathbf{R}_{Y Y}\right)\right]$ of $p\left(\mathbf{Y} \mid \overline{\mathbf{S}}^{i}\right)$ in (6) as well as the denominator $\prod_{\bar{k}=1}^{\left(N_{w}-1\right) \mathrm{BPS}}\left\{1+\exp \left[L_{a}\left(b_{\bar{k}}\right)\right]\right\}$ in the a priori probability of $p\left(\overline{\mathbf{S}}^{i}\right)=\frac{\exp \left[\sum_{\bar{k}=1}^{\left(N_{w}-1\right) \mathrm{BPS}} \widetilde{b}_{\bar{k}} L_{a}\left(b_{\bar{k}}\right)\right]}{\prod_{\bar{k}=1}^{\left(N_{w}-1\right) \mathrm{BPS}}\left\{1+\exp \left[L_{a}\left(b_{\bar{k}}\right)\right]\right\}}$ according to the LLR definition of $L_{a}=\ln \frac{p(b=1)}{p(b=0)}$ [37], [38].
}

eliminated by the the division of the Log-MAP of (14). However, in order to avoid excessive calculations in logarithm domain, we adopt the method in [34], [40], which uses a simple operation of $\bar{C}_{a, \bar{k}_{v}}=\frac{1}{2}\left[\left|L_{a}\left(b_{\bar{k}_{v}}\right)\right|+L_{a}\left(b_{\bar{k}_{v}}\right)\right]$ to guarantee a nonnegative ED. As a result, the PED of soft-decision MSDSD may be defined as [3]:

$$
\begin{aligned}
d_{v} & =\sum_{\bar{v}=2}^{v}\left\|\sum_{t=1}^{\bar{v}} \bar{s}_{t}^{*} \mathbf{U}_{t, \bar{v}}\right\|^{2}-\sum_{\bar{v}=2}^{v} \sum_{\bar{k}_{\bar{v}}=1}^{\text {BPS }}\left[\widetilde{b}_{\bar{k}_{\bar{v}}} L_{a}\left(b_{\bar{k}_{\bar{v}}}\right)-\bar{C}_{a, \bar{k}_{\bar{v}}}\right] \\
& =d_{v-1}+\Delta_{v-1},
\end{aligned}
$$

where the PED increment is given by:

$$
\begin{aligned}
\Delta_{v-1}=\| \bar{s}_{v-1}^{*} \mathbf{U}_{v, v}+x^{m} & \left(\sum_{t=1}^{v-1} \bar{s}_{t}^{*} \mathbf{U}_{t, v}\right) \|^{2} \\
& -\sum_{\bar{k}_{v}=1}^{\mathrm{BPS}}\left[\tilde{b}_{\bar{k}_{v}} L_{a}\left(b_{\bar{k}_{v}}\right)-\bar{C}_{a, \bar{k}_{v}}\right] .
\end{aligned}
$$

As introduced in [3], the Schnorr-Euchner search strategy may search for $x_{v-1}$ according to $\Delta_{v-1}$ of (19). However, unlike the hard-decision MSDSD, the decision variable $z_{v-1}$ of (13) cannot be directly used, because the a priori information $\sum_{\bar{k}_{v}=1}^{\mathrm{BPS}}\left[\widetilde{b}_{\bar{k}_{v}} L_{a}\left(b_{\bar{k}_{v}}\right)-\bar{C}_{a, \bar{k}_{v}}\right]$ is not included in $z_{v-1}$. As a result, the conventional Schnorr-Euchner search strategy in [3] has to visit all MPSK constellation points for $x_{v-1}$ by evaluating and sorting a total of $M$ PED increment values $\Delta_{v-1}$ of (19).

The soft-decision MSDSD algorithm based on the PED of (18) may now be implemented by the "MAP-MSDSD" function in [3], which is exemplified for the cases of employing DQPSK and D8PSK in Figs. 1 and 2, respectively. In summary, the MSDSD algorithm in [3] is capable of finding both the global minimum $d_{M A P}$ as well as the optimum constellation points $\left\{\hat{x}_{v-1}\right\}_{v=2}^{N_{w}-1}$, which may be translated into the hard-bit decisions of $\left\{b_{k}^{M A P}\right\}_{k=1}^{\left(N_{w}-1\right) \mathrm{BPS}}$. In order to produce soft-bit decisions, the Max-Log-MAP algorithm of (15) may be completed as:

$$
L_{p}\left(b_{k}\right)= \begin{cases}-d_{M A P}+\bar{d}_{M A P}, & \text { if } b_{k}^{M A P}=1 \\ -\bar{d}_{M A P}+d_{M A P}, & \text { if } b_{k}^{M A P}=0\end{cases}
$$

where $\bar{d}_{M A P}$ is obtained by invoking the MSDSD again, where the search space is halved by fixing $b_{k}$ to be the flipped version of the MAP decision as $b_{k}=\bar{b}_{k}^{M A P}$. In summary, when the consecutive MSDSD windows are simply overlapped by $N_{O L}=1$ observations, the MSDSD algorithm of [3] has to be invoked once first for finding the global MAP solution $d_{M A P}$ in (20), and then it is invoked for an additional $\left(N_{w}-1\right) \log _{2} M$ number of times for finding the local MAP solutions $\bar{d}_{M A P}$ in (20), which may be referred to as the Repeated Tree Search (RTS).

Alternatively, it's recently proposed in [33], [34] that the Single Tree Search (STS) [41] may opt to invoke the SD only once for obtaining all the EDs of $d_{M A P}$ and $\bar{d}_{M A P}$, which may induce a potential performance loss. More explicitly, if the hypothesis bit-mapping arrangement for $d_{M A P}$ is updated and changed, all the counter-hypothesis bit-mapping arrangements for $\bar{d}_{M A P}$ have to be changed accordingly. As a result, the previously dismissed candidates that obey the new bit-mapping cannot be taken into account again. As a remedy, the sub-optimal 

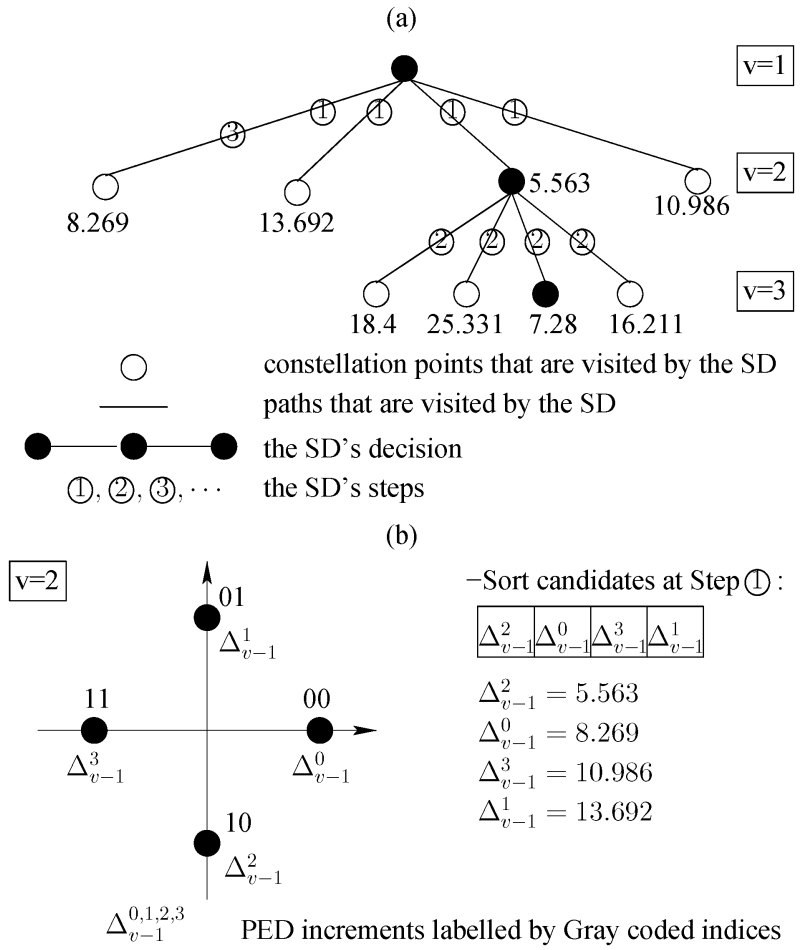

Fig. 1. Example of soft-decision MSDSD aided DQPSK, recorded at SNR = $0 \mathrm{~dB}$, where we have $I_{A}=0.3, N_{R}=2$ and $N_{w}=3$. (a) Example of Soft-Decision MSDSD Conceived for DQPSK Detection (labelled with PED values). (b) Example of QPSK constellation digram visited by Soft-Decision $\mathrm{SD}$ at index $\mathrm{v}=2$.

(a)

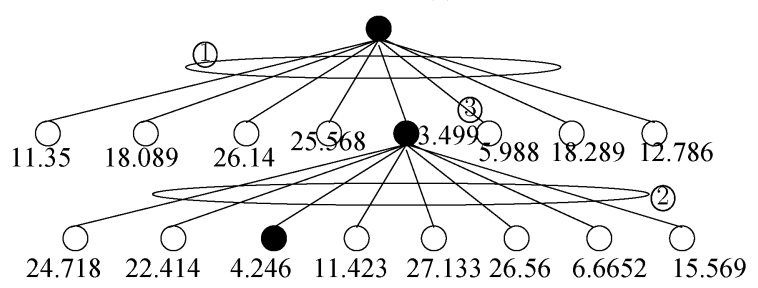

$\mathrm{v}=1$

$\mathrm{v}=2$

$\mathrm{v}=3$

(b)

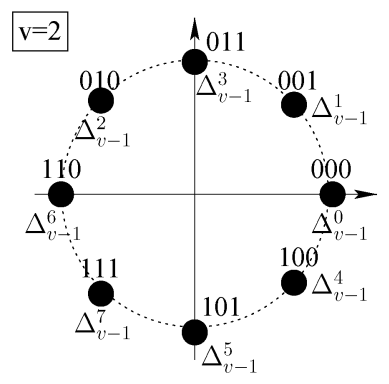

-Sort candidates at Step (1):

\begin{tabular}{|l|l|l|l|l|l|l|l|}
\hline$\Delta_{v-1}^{4}$ & $\Delta_{v-1}^{5}$ & $\Delta_{v-1}^{0}$ & $\Delta_{v-1}^{7}$ & $\Delta_{v-1}^{1}$ & $\Delta_{v-1}^{6}$ & $\Delta_{v-1}^{3}$ & $\Delta_{v-1}^{2}$ \\
\hline
\end{tabular}

$$
\begin{aligned}
& \Delta_{v-1}^{4}=3.499 \\
& \Delta_{v-1}^{5}=5.988 \\
& \Delta_{v-1}^{0}=11.35 \\
& \Delta_{v-1}^{7}=12.786 \\
& \Delta_{v-1}^{1}=18.089 \\
& \Delta_{v-1}^{6}=18.289 \\
& \Delta_{v-1}^{3}=25.568 \\
& \Delta_{v-1}^{2}=26.14
\end{aligned}
$$

Fig. 2. Example of soft-decision MSDSD aided D8PSK, recorded at SNR = $3 \mathrm{~dB}$, where we have $I_{A}=0.3, N_{R}=2$ and $N_{w}=3$. (a) Example of SoftDecision MSDSD Conceived for D8PSK Detection. (b) Example of 8PSK constellation digram visited by Soft-Decision SD at index v $=2$.

detector has to invoke the LLR correction method [34] for correcting the LLR results. Against this background, the RTS is suggested in this paper. In fact, the STS's motivation of visiting a node at most once can still be accomplished by the RTS, where the previously visited nodes may be labelled so that the repeated calculations may be avoided by reading the previously evaluated PED metrics.

\section{REDUCED-COMPLEXITY SOFT-DeCISION MSDSD}

It was demonstrated in Section III that the conventional Schnorr-Euchner search strategy utilized by the soft-decision MSDSD of [3] has to visit all MPSK constellation points. In this section, we opt to propose a reduced-complexity soft-decision MSDSD algorithm, where the Schnorr-Euchner search strategy may once again visit the MPSK constellation points in a zigzag fashion. More explicitly, the PED increment $\Delta_{v-1}$ of (19) may be further extended as:

$$
\begin{aligned}
& \Delta_{v-1}=\left\|\mathbf{A}_{v-1}^{\mathrm{MSDD}}-x_{v-1} \mathbf{B}_{v-1}^{\mathrm{MSDD}}\right\|^{2}-\sum_{\bar{k}_{v}=1}^{\mathrm{BPS}}\left[\widetilde{b}_{\bar{k}_{v}} L_{a}\left(b_{\bar{k}_{v}}\right)-\bar{C}_{a, \bar{k}_{v}}\right] \\
& =-2 \Re\left(\bar{x}_{v-1}^{*} \bar{z}_{v-1}\right)-\sum_{\bar{k}_{v}=1}^{\mathrm{BPS}} \widetilde{b}_{\bar{k}_{v}} L_{a}\left(b_{\bar{k}_{v}}\right)+\frac{L_{a}\left(b_{1}\right)+L_{a}\left(b_{2}\right)}{2}+\bar{C}_{v-1},
\end{aligned}
$$

where we deliberately rotate all the detected MPSK constellations (except for BPSK $^{4}$ ) anti-clockwise by $(\pi / M)$ as suggested by [28], i.e. we have $\bar{x}_{v-1}=x_{v-1} \cdot \exp \left(j \frac{\pi}{M}\right)$, so that there are exactly $M / 4$ constellation points in each quadrant. Furthermore, the new decision variable $\bar{z}_{v}-1$ seen in (21) is given by:

$$
\bar{z}_{v-1}=\mathbf{A}_{v-1}^{\mathrm{MSDD}}\left(\mathbf{B}_{v-1}^{\mathrm{MSDD}}\right)^{H} \cdot \exp \left(j \frac{\pi}{M}\right)
$$

which is rotated anti-clockwise from the correlation decision variable $z_{v-1}^{M S D D}$ of (13) by $(\pi / M)$ for detecting $\bar{x}_{v-1}$, while the constant of $\bar{C}_{v-1}$ seen in (21) is given by:

$$
\bar{C}_{v-1}=\left\|\mathbf{A}_{v-1}^{\mathrm{MSDD}}\right\|^{2}+\left\|\mathbf{B}_{v-1}^{\mathrm{MSDD}}\right\|^{2}+\bar{C}_{a, v-1}-\frac{L_{a}\left(b_{1}\right)+L_{a}\left(b_{2}\right)}{2},
$$

and we have the constant $\bar{C}_{a, v-1}=\sum_{\bar{k}_{v}=1}^{\mathrm{BPS}} \bar{C}_{a, \bar{k}_{v}}$. We note that $\bar{C}_{v-1}$ of (23) is invariant over all the different candidates $\bar{x}_{v-1}$ in (21). As a result, comparing the $M$ candidates $\left\{x^{m}\right\}_{m=0}^{M-1}$ according to their PED increment values $\Delta_{v-1}$ of (21) is equivalent to comparing the following equivalent PED increment metric over the variables $\bar{x}_{v-1}$ as:

$$
\begin{aligned}
\bar{\Delta}_{v-1}= & -2 \Re\left(\bar{x}_{v-1}\right) \Re\left(\bar{z}_{v-1}\right)-2 \Im\left(\bar{x}_{v-1}\right) \Im\left(\bar{z}_{v-1}\right) \\
& -\sum_{\bar{k}_{v}=1}^{\mathrm{BPS}} \widetilde{b}_{\bar{k}_{v}} L_{a}\left(b_{\bar{k}_{v}}\right)+\frac{L_{a}\left(b_{1}\right)+L_{a}\left(b_{2}\right)}{2},
\end{aligned}
$$

where we have the algebraic relationship of $\Re\left(\bar{x}_{v-1}^{*} \bar{z}_{v-1}\right)=$ $\Re\left(\bar{x}_{v-1}\right) \Re\left(\bar{z}_{v-1}\right)+\Im\left(\bar{x}_{v-1}\right) \Im\left(\bar{z}_{v-1}\right)$.

Considering the rotated QPSK as an example, the four probability metrics $\left\{\bar{\Delta}_{v-1}^{m}\right\}_{m=0}^{M-1=3}$ of (24) corresponding to the $M=4$ rotated QPSK constellation points $\left\{\bar{x}^{m}= \pm \frac{1}{\sqrt{2}} \pm j \frac{1}{\sqrt{2}}\right\}_{m=0}^{M-1=3}$ may be expressed as (25), shown at the bottom of the next page.

\footnotetext{
${ }^{4}$ We note that rotating the BPSK constellation anti-clockwise by $(\pi / M)$ will only move the two BPSK constellation points from the real axis to the imaginary axis, which is not neccessary.
} 
TABLE I

PSEUdo-Code For REDUCED-COMPLEXITY SOFT-DECISION MSDSD AIDEd DPSK

\begin{tabular}{|c|c|c|}
\hline Function: & \multicolumn{2}{|c|}{$\begin{array}{c}{\left[\left\{\hat{x}_{v-1}\right\}_{v=2}^{N_{w}}, d_{M A P}\right]=\text { Soft-MSDSD-RC }\left(\left\{\left\{\mathbf{U}_{t, v}\right\}_{t=1}^{v}\right\}_{v=1}^{N_{w}},\left\{\left\{L_{a}^{v-1, k}\right\}_{v=2}^{N_{w}}\right\}_{k=1}^{2},\left\{\bar{C}_{a, v-1}\right\}_{v=2}^{N_{w}}\right.} \\
\left.\left\{\left\{P_{v-1}^{g}\right\}_{g=0}^{M / 4-1}\right\}_{v=2}^{N_{w}}, N_{w}, R^{2}\right)\end{array}$} \\
\hline 1: $d_{1}=0$ & & //initialize PED \\
\hline 2: $\bar{s}_{1}=1$ & & //initialize the first transmitted symbol \\
\hline 3: $v=2$ & & //initialize SD search index \\
\hline \multirow{2}{*}{\multicolumn{3}{|c|}{$\begin{array}{l}\text { 4: (subfunction) findBest-DBPSK/findBest-DQPSK/findBest-DPSK } \\
\text { 5: loop }\end{array}$}} \\
\hline & & \\
\hline \multicolumn{3}{|c|}{ 6: $\quad d_{v}=d_{v-1}+\bar{\Delta}_{v-1}+\bar{C}_{v-1}$} \\
\hline \multicolumn{3}{|r|}{ //update candidate data symbol } \\
\hline \multicolumn{3}{|r|}{ //update candidate transmitted symbol } \\
\hline \multicolumn{3}{|c|}{ if $v \neq N_{w}$} \\
\hline \multirow{2}{*}{\multicolumn{3}{|c|}{ (subfunction) findBest-DBPSK/findBest-DQPSK/findBest-DPSK }} \\
\hline & & \\
\hline \multicolumn{3}{|l|}{ else } \\
\hline \multicolumn{3}{|r|}{ //update $\mathrm{SD}$ radius } \\
\hline \multicolumn{3}{|c|}{$\begin{array}{l}\left\{\hat{x}_{v-1}\right\}_{v=2}^{N_{w}^{w}}=\left\{x_{v-1}\right\}_{v=2}^{N_{w}} \\
\text { do }\end{array}$} \\
\hline $\begin{array}{l}17: \\
18:\end{array}$ & if $v==2$ return $\left[\left\{\hat{x}_{v-1}\right\}_{v=2}^{N_{w}}, R^{2}\right]$ and exit & $\begin{array}{l}\text { //terminate SD for the case of } N_{w}=2 \\
/ / \text { move down }\end{array}$ \\
\hline \multicolumn{3}{|c|}{ while $n_{v-1}==(M-1)$} \\
\hline \multicolumn{3}{|c|}{ (subfunction) findNext-DBPSK/findNext-DQPSK/findNext-DPSK } \\
\hline \multirow{2}{*}{\multicolumn{3}{|c|}{ 21: end if }} \\
\hline \multirow{2}{*}{\multicolumn{3}{|c|}{$\begin{array}{ll}\text { 22: } & \text { else } \\
\text { 23: } & \text { do }\end{array}$}} \\
\hline & & \\
\hline \multicolumn{2}{|r|}{$v=v-1$} & \\
\hline \multicolumn{3}{|c|}{ while $n_{v-1}==(M-1)$} \\
\hline \multicolumn{3}{|c|}{ 7: (subfunction) findNext-DBPSK/findNext-DQPSK/findNext-DPSK } \\
\hline $\begin{array}{l}\text { 28: end if } \\
\text { 29: end loop }\end{array}$ & & \\
\hline
\end{tabular}

where we associate the real and imaginary parts of $\bar{z}_{v-1}$ with $L_{a}\left(b_{2}\right)$ and $L_{a}\left(b_{1}\right)$ respectively as:

$t_{v-1}^{R e}=\sqrt{2} \Re\left(\bar{z}_{v-1}\right)-\frac{L_{a}\left(b_{2}\right)}{2}, \quad t_{v-1}^{I m}=\sqrt{2} \Im\left(\bar{z}_{v-1}\right)-\frac{L_{a}\left(b_{1}\right)}{2}$.

After assigning the a priori LLRs to the appropriate parts of $\bar{z}_{v-1}$, the only difference between the four candidates $\left\{\bar{\Delta}_{v-1}^{m}\right\}_{m=0}^{M-1=3}$ in (25) is the polarity of $t_{v-1}^{R e}$ and $t_{v-1}^{I m}$. This allows us to directly obtain the minimum metric by simply evaluating $\bar{\Delta}_{v-1}=-\left|t_{v-1}^{R e}\right|-\left|t_{v-1}^{I m}\right|$, and then the ranking order of the rest of the candidates may be obtained by comparing $\left|t_{v-1}^{R e}\right|$ and $\left|t_{v-1}^{I m}\right|$. In more detail, if we have the condition of $\left|t_{v-1}^{R e}\right|>$ $\left|t_{v-1}^{I m}\right|$, then the SD may visit the remaining candidates in a zigzag fashion according to the steps of $\bar{\Delta}_{v-1}=-\left|t_{v-1}^{R e}\right|+\left|t_{v-1}^{I m}\right|$, $\bar{\Delta}_{v-1}=\left|t_{v-1}^{R e}\right|-\left|t_{v-1}^{I m}\right|$ and $\bar{\Delta}_{v-1}=\left|t_{v-1}^{R e}\right|+\left|t_{v-1}^{I m}\right|$. Other- wise, the remaining steps should be $\bar{\Delta}_{v-1}=\left|t_{v-1}^{R e}\right|-\left|t_{v-1}^{I m}\right|$, $\bar{\Delta}_{v-1}=-\left|t_{v-1}^{R e}\right|+\left|t_{v-1}^{I m}\right|$ and $\bar{\Delta}_{v-1}=\left|t_{v-1}^{R e}\right|+\left|t_{v-1}^{I m}\right|$. In summary, similar to the condition of $\operatorname{sign}\left(p_{v-1}-\left\lfloor p_{v-1}\right\rceil\right)$ for the hard-decision MSDSD of Section II, the soft-decision MSDSD aided DQPSK may rely on the condition of $\operatorname{sign}\left(\left|t_{v-1}^{R e}\right|-\right.$ $\left.\left|t_{v-1}^{I m}\right|\right)$ for deciding the direction of SD's zigzag path.

In more detail, the reduced-complexity soft-decision MSDSD is summarized in the form of its pseudo-code in Table I, where the simplified Schnorr-Euchner search strategy specifically tailored for DBPSK and DQPSK is given by Tables II and III, respectively. Furthermore, Fig. 3 revisits the specific example of Fig. 1, where the reduced-complexity algorithms of Tables I and III are invoked. It can be seen in Fig. 3 that the proposed reduced-complexity MSDSD exhibits a reduced number of visited nodes compared to the conventional MSDSD exemplified by Fig. 1.

$$
\begin{aligned}
& \bar{\Delta}_{v-1}^{0}=-\sqrt{2} \Re\left(\bar{z}_{v-1}\right)-\sqrt{2} \Im\left(\bar{z}_{v-1}\right)+\frac{L_{a}\left(b_{1}\right)+L_{a}\left(b_{2}\right)}{2}=-t_{v-1}^{R e}-t_{v-1}^{I m}, \\
& \bar{\Delta}_{v-1}^{1}=\sqrt{2} \Re\left(\bar{z}_{v-1}\right)-\sqrt{2} \Im\left(\bar{z}_{v-1}\right)-L_{a}\left(b_{2}\right)+\frac{L_{a}\left(b_{1}\right)+L_{a}\left(b_{2}\right)}{2}=t_{v-1}^{R e}-t_{v-1}^{I m}, \\
& \bar{\Delta}_{v-1}^{2}=-\sqrt{2} \Re\left(\bar{z}_{v-1}\right)+\sqrt{2} \Im\left(\bar{z}_{v-1}\right)-L_{a}\left(b_{1}\right)+\frac{L_{a}\left(b_{1}\right)+L_{a}\left(b_{2}\right)}{2}=-t_{v-1}^{R e}+t_{v-1}^{I m}, \\
& \bar{\Delta}_{v-1}^{3}=\sqrt{2} \Re\left(\bar{z}_{v-1}\right)+\sqrt{2} \Im\left(\bar{z}_{v-1}\right)-L_{a}\left(b_{1}\right)-L_{a}\left(b_{2}\right)+\frac{L_{a}\left(b_{1}\right)+L_{a}\left(b_{2}\right)}{2}=t_{v-1}^{R e}+t_{v-1}^{I m},
\end{aligned}
$$


TABLE II

PSEUdo-Code For THE SUBFUNCTIONS OF THE REDUCED-COMPLEXITY SOFT-DECISION MSDSD OF TABLE I, WHERE DBPSK IS EMPLOYED

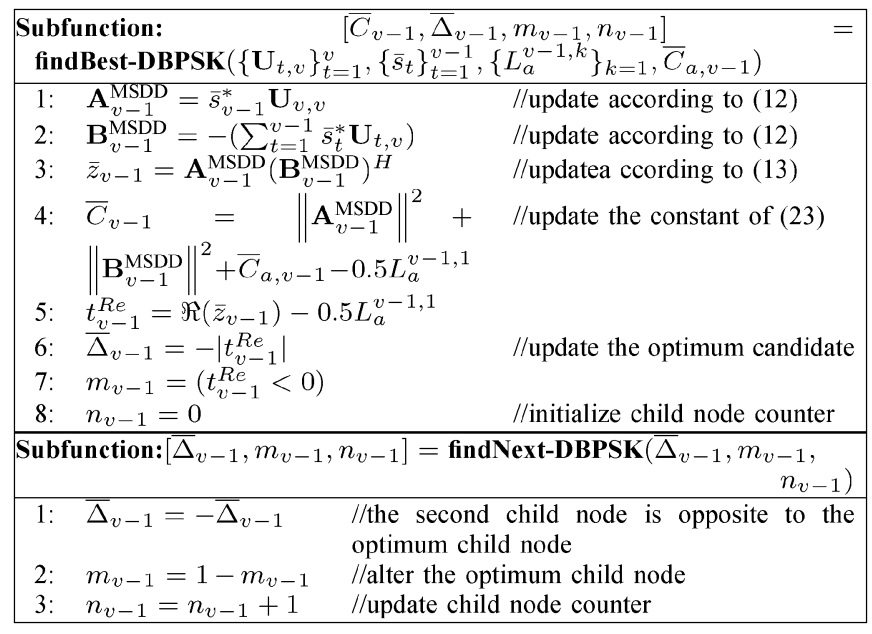

Let us now consider the rotated 8PSK constellation portrayed by Fig. 4(b) as an example, where the $M=8$ constellation points are arranged to $M / 4=2$ groups, which are Group G0 of $\left\{ \pm \cos \left(\frac{\pi}{8}\right) \pm j \sin \left(\frac{\pi}{8}\right)\right\}$ and Group G1 of $\left\{ \pm \sin \left(\frac{\pi}{8}\right) \pm\right.$ $\left.j \cos \left(\frac{\pi}{8}\right)\right\}$. Accordingly, their probability metrics $\left\{\bar{\Delta}_{v-1}^{m}\right\}_{m=0}^{M-1=7}$ of (24) may be expressed as:

$$
\begin{aligned}
& \bar{\Delta}_{v-1}^{0}=-t_{v-1}^{R e 0}-t_{v-1}^{I m 0}, \quad \bar{\Delta}_{v-1}^{2}=t_{v-1}^{R e 0}-t_{v-1}^{I m 0}, \\
& \bar{\Delta}_{v-1}^{4}=-t_{v-1}^{R e 0}+t_{v-1}^{I m 0}, \quad \bar{\Delta}_{v-1}^{6}=t_{v-1}^{R e 0}+t_{v-1}^{I m 0}, \\
& \bar{\Delta}_{v-1}^{1}=-t_{v-1}^{R e 1}-t_{v-1}^{I m 1}-L_{a}\left(b_{3}\right), \\
& \bar{\Delta}_{v-1}^{3}=t_{v-1}^{R e 1}-t_{v-1}^{I m 1}-L_{a}\left(b_{3}\right), \\
& \bar{\Delta}_{v-1}^{5}=-t_{v-1}^{R e 1}+t_{v-1}^{I m 1}-L_{a}\left(b_{3}\right), \\
& \bar{\Delta}_{v-1}^{7}=t_{v-1}^{R e 1}+t_{v-1}^{I m 1}-L_{a}\left(b_{3}\right),
\end{aligned}
$$

where the two pairs of real/imaginary terms are given by $t_{v-1}^{R e 0}=$ $2 \cos \left(\frac{\pi}{8}\right) \cdot \Re\left(\bar{z}_{v-1}\right)-\frac{L_{a}\left(b_{2}\right)}{2}, t_{v-1}^{I m 0}=2 \sin \left(\frac{\pi}{8}\right) \cdot \Im\left(\bar{z}_{v-1}\right)-\frac{L_{a}\left(b_{1}\right)}{2}$, $t_{v-1}^{R e 1}=2 \sin \left(\frac{\pi}{8}\right) \cdot \Re\left(\bar{z}_{v-1}\right)-\frac{L_{a}\left(b_{2}\right)}{2}$ and $t_{v-1}^{\operatorname{Im} 1}=2 \cos \left(\frac{\pi}{8}\right) \cdot \Im\left(\bar{z}_{v}-1\right)-$ $\frac{L_{a}\left(b_{1}\right)}{2}$. It can be seen in (27a) and (27b) that the only difference between the four component probability metrics within each group is the polarity of the real/imaginary terms. As a result, the local minimum metrics of the $M / 4=2$ groups may be obtained by $\bar{\Delta}_{v-1}^{G 0}=\min _{m \in\{0,2,4,6\}} \bar{\Delta}_{v-1}^{m}=-\left|t_{v-1}^{R e 0}\right|-\left|t_{v-1}^{I m 0}\right|$ and $\bar{\Delta}_{v-1}^{G 1}=\min _{m \in\{1,3,5,7\}} \bar{\Delta}_{v-1}^{m}=-\left|t_{v-1}^{R e 1}\right|-\left|t_{v-1}^{I m 1}\right|-L_{a}\left(b_{3}\right)$, respectively, which are evaluated without invoking (24) for $M=8$ times in (27). Finally, the global minimum over $\left\{\bar{\Delta}_{v-1}^{m}\right\}_{m=0}^{M-1=7}$ of (24) may be simply obtained by comparing the two local minima as $\bar{\Delta}_{v-1}=\min \left\{\bar{\Delta}_{v-1}^{G 0}, \bar{\Delta}_{v-1}^{G 1}\right\}$.

In summary, for a generic high-order MPSK scheme $(M>4)$, we may firstly assign the $M$ constellation points to $M / 4$ groups of QPSK-like constellation points that are associated with the same magnitudes but different polarities, so that the local minimum metric for $\left\{\bar{\Delta}_{v-1}^{m}\right\}_{m=0}^{M-1}$ of (24) within each group is simply given by:

$$
\bar{\Delta}_{v-1}^{G g}=-\left|t_{v-1}^{R e_{g}}\right|-\left|t_{v-1}^{I m_{g}}\right|-\sum_{\bar{k}=3}^{\mathrm{BPS}} \widetilde{b}_{\bar{k}} L_{a}\left(b_{\bar{k}}\right),
$$

where the range for the group index is given by $g \in\{0, \cdots$, $M / 4-1\}$, while the real and imaginary parts of $\bar{z}_{v-1}$ are associated with $L_{a}\left(b_{2}\right)$ and $L_{a}\left(b_{1}\right)$ respectively as follows:

$t_{v-1}^{R e_{g}}=\bar{A}^{g} \cdot \Re\left(\bar{z}_{v-1}\right)-\frac{L_{a}\left(b_{2}\right)}{2}, \quad t_{v-1}^{I m_{g}}=\bar{B}^{g} \cdot \Im\left(\bar{z}_{v-1}\right)-\frac{L_{a}\left(b_{1}\right)}{2}$.

The coordinates of the MPSK constellation points, which are located in the first quadrant may be denoted by $\left\{\left(A^{g}\right.\right.$, $\left.\left.B^{g}\right)\right\}_{g=0}^{M / 4-1}$, and we have $\bar{A}^{g}=2 A^{g}$ as well as $\bar{B}^{g}=2 B^{g}$ in (29). As a result, the global minimum for $\left\{\bar{\Delta}_{v-1}^{m}\right\}_{m=0}^{M-1}$ of (24) may be simply given by:

$$
\bar{\Delta}_{v-1}=\min _{g \in\{0, \cdots, M / 4-1\}} \bar{\Delta}_{v-1}^{G g},
$$

which is obtained by visiting a reduced-subset of $M / 4$ constellation points that correspond to the $M / 4$ local minima of (28).

We note that the procedures conceived for obtaining the minimum probability metric of (30) are similar to those in our previous work designed for generic soft-decision MPSK detection in [28]. However, for the soft-decision MSDSD, the Schnorr-Euchner search strategy also relies on the ranking order of constellation points. Against this background, we propose to complete the Schnorr-Euchner strategy by using a Comparison Window (CW). More explicitly, the $\mathrm{CW}$ is initialized to have $M / 4$ local minima of (28), which correspond to local best candidates. Then the $\mathrm{CW}$ may choose the global best candidate which has the global minimum metric by invoking (30). This is the subfunction of "findBest-DPSK" for the Schnorr-Euchner search strategy tailored for MPSK $(M>4)$ in Table IV. Furthermore, when the SD re-visits a specific SD index $v$, the "findNext-DPSK" subfunction in Table IV may offer the next constellation node. More explicitly, if previously a local minimum from Group $\mathrm{G} \bar{g}$ is chosen as the global candidate, i.e. previously we have $\bar{\Delta}_{v-1}=\bar{\Delta}_{v-1}^{G \bar{g}}$ from (30), then Group G $\bar{g}$ has to visit a new local candidate in a zigzag fashion by comparing $\left|t_{v-1}^{R e_{\bar{g}}}\right|$ and $\left|t_{v-1}^{I m_{\bar{g}}}\right|$. Following this, the CW may once again update the new global candidate by invoking (30).

Fig. 4 portrays the D8PSK example of Fig. 2, where the reduced-complexity algorithms of Tables I and IV are invoked. More explicitly, it can be seen in Fig. 4 that the "findBestDPSK" subfunction in Table IV may firstly initialize the CW by the $M / 4=2$ local minima of (28) as $\bar{\Delta}_{v-1}^{G 0}=-3.487$ and $\bar{\Delta}_{v-1}^{G 1}=-0.998$, and then the CW invokes (30) in order to obtain the global candidate of $\bar{\Delta}_{v-1}=\bar{\Delta}_{v-1}^{G 0}=-3.487$. Moreover, when the SD re-visits index $v=2$ in Fig. 4, the "findNextDPSK" in Table IV may firstly update a new local candidate $\bar{\Delta}_{v-1}^{G 0}=4.364$ from Group G0 by visiting the QPSK-like constellation points in a zigzag fashion relying on the relationship between $\left|t_{v-1}^{R e 0}\right|$ and $\left|t_{v-1}^{I m 0}\right|$, and then the CW invokes (30) again in order to obtain the new global candidate of $\bar{\Delta}_{v-1}=\bar{\Delta}_{v-1}^{G 1}=$ -0.998 . As a result, the reduced-complexity MSDSD exemplified in Fig. 4 visits a reduced subset of the constellation points compared to the conventional MSDSD exemplified in Fig. 2, yet the same SD result is arrived at.

Moreover, it is worth pointing out that the conventional MSDSD algorithm in [3] requires the Schnorr-Euchner search 
TABLE III

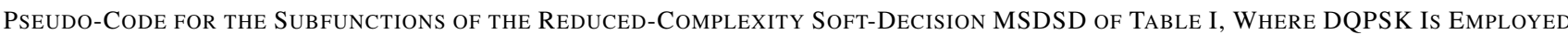

\begin{tabular}{|c|c|}
\hline \multicolumn{2}{|c|}{ Subfunction: $\left[\bar{C}_{v-1},\left|t_{v-1}^{R e}\right|,\left|t_{v-1}^{I m}\right|, \bar{\Delta}_{v-1}, m_{v-1}, n_{v-1}\right]=\mathbf{f i n d B e s t - D Q P S K}\left(\left\{\mathbf{U}_{t, v}\right\}_{t=1}^{v},\left\{\bar{s}_{t}\right\}_{t=1}^{v-1},\left\{L_{a}^{v-1, k}\right\}_{k=1}^{2}, \bar{C}_{a, v-1}\right)$} \\
\hline \multirow{2}{*}{\multicolumn{2}{|c|}{$\begin{array}{ll}1: & \mathbf{A}_{v-1}^{\mathrm{MSDD}}=\bar{s}_{v-1}^{*} \mathbf{U}_{v, v} \\
2: & \mathbf{B}_{v-1}^{\mathrm{MSDD}}=-\left(\sum_{t-1}^{v-1} \bar{s}_{+}^{*} \mathbf{U}_{t, v}\right)\end{array}$}} \\
\hline & //update $\mathbf{B}_{v-1}^{\mathrm{MSDD}}$ according to Eq. (12) \\
\hline \multicolumn{2}{|l|}{ 3: $\quad \bar{z}_{v-1}^{M-1} \overline{S S D}=\mathbf{A}_{v-1}^{\mathrm{MSDD}}\left(\mathbf{B}_{v-1}^{\mathrm{MSDD}}\right)^{H} \cdot \exp \left(j \frac{\pi}{M}\right)$} \\
\hline \multicolumn{2}{|c|}{ 4: $\quad \bar{C}_{v-1}=\left\|\mathbf{A}_{v-1}^{\mathrm{MSDD}}\right\|^{2}+\left\|\mathbf{B}_{v-1}^{\mathrm{MSDD}}\right\|^{2}+\bar{C}_{a, v-1}-0.5\left(L_{a}^{v-1,1}+L_{a}^{v-1,2}\right)$} \\
\hline \multicolumn{2}{|l|}{ 5: $\quad t_{v-1}^{R e}=\sqrt{2} \Re\left(\bar{z}_{v-1}^{M S} D S D\right)-0.5 L_{a}^{v-1,2}$} \\
\hline \multicolumn{2}{|l|}{ 6: $\quad t_{v-1}^{I m}=\sqrt{2} \Im\left(\bar{z}_{v-1}^{M S D S D}\right)-0.5 L_{a}^{v-1,1}$} \\
\hline \multicolumn{2}{|l|}{ 7: $\quad \bar{\Delta}_{v-1}=-\left|t_{v-1}^{R e}\right|-\left|t_{v-1}^{I m}\right|$} \\
\hline \multicolumn{2}{|r|}{ //update the optimum candidate } \\
\hline \multicolumn{2}{|l|}{ 9: $\quad b_{2}=\left(t_{v-1}^{R-1}<0\right)$} \\
\hline \multicolumn{2}{|r|}{ //translate binary bits to decimal index } \\
\hline \multirow{2}{*}{\multicolumn{2}{|c|}{$\begin{array}{lr}\text { 11: } n_{v-1}=0 & \text { //initialize child node counter } \\
\text { Subfunction: } & {\left[\bar{\Delta}_{v-1}, m_{v-1}, n_{v-1}, \operatorname{cd}_{v-1}\right]=\text { findNext-DQPSK }\left(\left|t^{R e}\right|,\left|t^{I m}\right|, \bar{\Delta}_{v-1}, m_{v-1}, n_{v-1}, \operatorname{cd}_{v-1}\right)}\end{array}$}} \\
\hline & \\
\hline \multicolumn{2}{|c|}{$1: \quad n_{v-1}=n_{v-1}+1 \quad$ //update child node counter } \\
\hline \\
\hline \multicolumn{2}{|l|}{ 3: $\quad$ case $1: b_{1} b_{2}=\operatorname{dec} 2 \operatorname{bin}\left(m_{v-1}\right)$} \\
\hline 4: $\quad \operatorname{cd}_{v-1}=\operatorname{sign}\left(\left|t_{v-1}^{R e}\right|-\left|t_{v-1}^{I m}\right|\right)$ & //update the condition \\
\hline if $\mathrm{cd}_{v-1}==1$ & $/ /$ the case of $\left|t_{v-1}^{R e}\right|>\left|t_{v-1}^{I m}\right|$ \\
\hline 6: $\quad \bar{\Delta}_{v-1}=-\left|t_{v-1}^{R e}\right|+\left|t_{v-1}^{I m}\right|$ & //alter the imaginary part of the PED increment \\
\hline$\quad m_{v-1}=\operatorname{bin} 2 \operatorname{dec}\left(\bar{b}_{1} b_{2}\right)$ & //alter $b_{1}$ in the optimum child node \\
\hline 8: $\quad$ else & $/ /$ the case of $\left|t_{v-1}^{R e}\right|<\left|t_{v-1}^{\operatorname{Im}}\right|$ \\
\hline $\bar{\Delta}_{v-1}=\left|t_{v-1}^{R e}\right|-\left|t_{v-1}^{I m}\right|$ & //alter the real part of the PED increment \\
\hline 10: $\quad m_{v-1}=\operatorname{bin} 2 \operatorname{dec}\left(b_{1} \bar{b}_{2}\right)$ & //alter $b_{2}$ in the optimum child node \\
\hline end if & \\
\hline \multicolumn{2}{|l|}{ 12: $\quad$ break } \\
\hline case $2: \bar{\Delta}_{v-1}=-\bar{\Delta}_{v-1}$ & //alter the decision made by the previous step \\
\hline$m_{v-1}=3-m_{v-1}$ & \\
\hline break & \\
\hline case 3: $b_{1} b_{2}=\operatorname{dec} 2 \operatorname{bin}\left(m_{v-1}\right)$ & \\
\hline $\bar{\Delta}_{v-1}=\left|t_{v-1}^{R e}\right|+\left|t_{v-1}^{I m}\right|$ & //alter the optimum child node \\
\hline if $\mathrm{cd}_{v-1}==1 m_{v-1}=\operatorname{bin} 2 \operatorname{dec}\left(\bar{b}_{1} b_{2}\right)$ & //alter $b_{1}$ in the decision made by the previous step \\
\hline else $m_{v-1}=\operatorname{bin} 2 \operatorname{dec}\left(b_{1} \bar{b}_{2}\right)$ & //alter $b_{2}$ in the decision made by the previous step \\
\hline 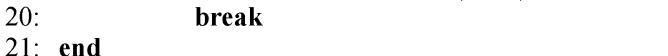 & \\
\hline
\end{tabular}

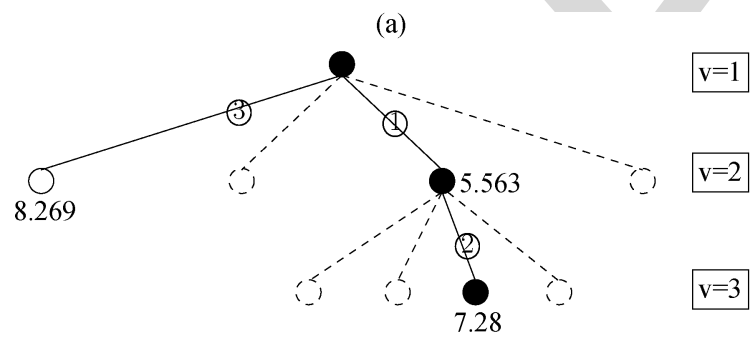

(1) the SD paths that are avoided

because of the reduced-complexity design

(b)

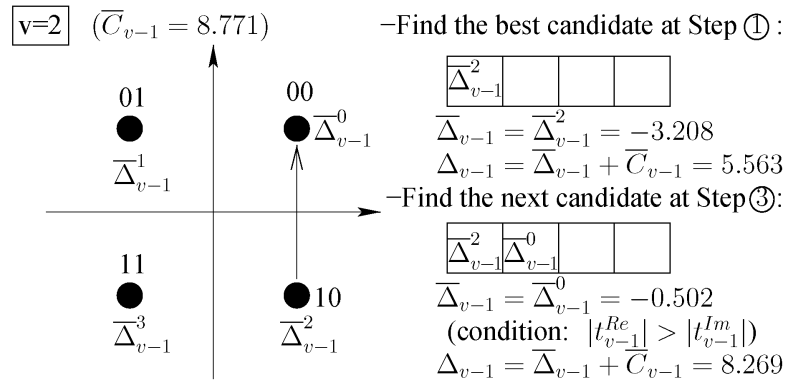

Fig. 3. Example of soft-decision MSDSD aided DQPSK, which corresponds to the example seen in Fig. 1, subject to the difference that the reducedcomplexity algorithm of Tables I and III are invoked. (a) Example of ReducedComplexity Soft-Decision MSDSD Conceived for DQPSK Detection. (b) Example of QPSK constellation diagram visited by Reduced-Complexity Soft-Decision SD at index $\mathrm{v}=2$. strategy to invoke a sorting algorithm, which was represented by the "qsort" function on line F-6 in the pseudo-code algorithm table of [3]. As a result, all $M$ constellation points $\left\{x^{m}\right\}_{m=0}^{M-1}$ are ranked according to an ascending order of the PED increment values $\left\{\Delta_{v-1}^{m}\right\}_{m=0}^{M-1}$, which is explicitly exemplified by Figs. 1 and 2. There are numerous sorting algorithms that may be suitable, such as Bubble sort, Timsort, Library sort [42], [43], etc., but the average number of comparisons required by these algorithms is as high as $O(M \log M)$. By contrast, the reduced-complexity MSDSD of Tables II-IV does not require any sorting algorithms. As exemplified by Figs. 3 and 4, the proposed Schnorr-Euchner search strategy does not have to maintain the complete ranking order of constellation points, which dispenses with a considerable number of comparisons.

\section{APPROX-LOG-MAP IMPLEMENTED BY MSDSD}

The soft-decision MSDSD discussed in the Sections III and IV aims to implement the Max-Log-MAP of (15), which may impose a performance loss compared to the near-optimum ApproxLog-MAP of (16). In order to mitigate this open problem, we propose the Approx-Log-MAP solution for MSDSD as follows:

(1) Let us define the leaf nodes of a SD structure as the candidates associated with the SD index $v=N_{w}$. For example, the $M=4$ candidates visited at the SD's step of (2) in Fig. 1(a) as well as the $M=8$ candidates visited at step (2) 
(a)

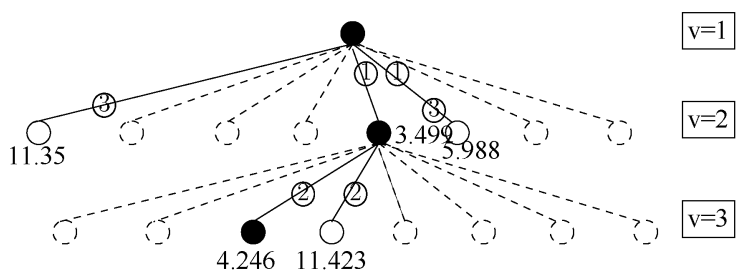

(b)

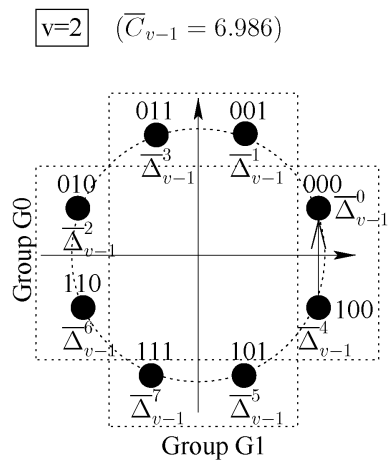

-Find the best candidate at Step (1)

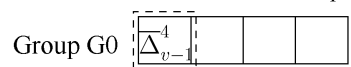

Group G1 \begin{tabular}{l|l|l|l|l|}
\hline$\Delta_{v-1}^{5}$ & & & \\
\hline
\end{tabular}

Comparison Window: $\bar{\Delta}_{v-1}^{G 0}=\bar{\Delta}_{v-1}^{4}=-3.487$ $\bar{\Delta}_{v-1}^{G 1}=\bar{\Delta}_{v-1}^{5}=-0.998$ $\therefore \bar{\Delta}_{v-1}=\overline{\min }_{\bar{\Delta}}\left\{\bar{\Delta}_{v-1}^{G 0}, \bar{\Delta}_{v-1}^{G 1}\right\}=-3.287$ $\therefore \Delta_{v-1}=\bar{\Delta}_{v-1}+\bar{C}_{v-1}=3.499$

-Find the next candidate at Step (3):

Group G1 \begin{tabular}{|l|l|l|l|}
\hline & $\Delta_{v-1}^{5}$ & & \\
\hline
\end{tabular} Comparison Window: $\bar{\Delta}_{v-1}^{G 0}=\bar{\Delta}_{v-1}^{0}=4.364$ (condition $\left|t_{v-1}^{R e 0}\right|>\left|t_{v-1}^{I m 0}\right|$ ) $\bar{\Delta}_{v-1}^{G 1}=\bar{\Delta}_{v-1}^{5}=-0.998$

$\therefore \bar{\Delta}_{v-1}=\min \left\{\bar{\Delta}_{v-1}^{G 0}, \bar{\Delta}_{v-1}^{G 1}\right\}=-0.998$

$\therefore \Delta_{v-1}=\bar{\Delta}_{v-1}+\bar{C}_{v-1}=5.988$

Fig. 4. Example of the soft-decision MSDSD aided D8PSK, which corresponds to the example seen in Fig. 2, subject to the difference that the reduced-complexity algorithms of Tables I and IV are invoked. (a) Example of Reduced-Complexity Soft-Decision MSDSD Conceived for D8PSK Detection. (b) Example of 8PSK constellation diagram visited by ReducedComplexity Soft-Decision SD at index v $=2$.

in Fig. 2(a) are all leaf nodes. This leads us to the proposed change of the MSDSD output scenario. When the MSDSD algorithm is invoked for the first time, instead of just producing the global optimum $d_{M A P}$ and the MAP hard-bit decisions $\left\{b_{k}^{M A P}\right\}_{k=1}^{\left(N_{w}-1\right) \mathrm{BPS}}$, all the PED values of the leaf nodes $\left\{d_{C A N}=d_{v}\right\}_{\forall v=N_{w}}$ as well as all the corresponding hardbit decisions $\left\{b_{k}^{C A N}\right\}_{k=1}^{\left(N_{w}-1\right) \mathrm{BPS}}$ may also be recorded and produced. For example, the SD of Fig. 1(a) may produce both the MAP solution, which is represented by $d_{M A P}=7.28$ and $\left\{b_{k}^{M A P}\right\}_{k=1}^{\left(N_{w}-1\right) \mathrm{BPS}}=1010$, as well as all the leaf nodes, which are represented by $\left\{d_{C A N}\right\}=\{18.4,25.331,7.28$, $16.211\}$ and $\left\{b_{k}^{M A P}\right\}_{k=1}^{\left(N_{w}-1\right) \mathrm{BPS}}=\{1000,1001,1010,1011\}$.

(2) For each soft-bit output, the MSDSD algorithm is invoked again with a fixed bit $b_{k}=\bar{b}_{k}^{M A P}$. Similarly, whenever the SD visits index $v=N_{w}$, the resultant $M$ leaf candidates $\left\{d_{C A N}^{b_{k}=\bar{b}_{k}^{M A P}}\right\}$ may all be recorded and produced. For example, when the SD of Fig. 1(a) is invoked again with a fixed bit $b_{1}=0$, the resultant SD structure is portrayed in Fig. 5, where the two sub-groups of leaf nodes $\{25.118$, $35.09,18.97,14.91\}$ and $\{20.05,24.09,46.615,35.05\}$ may be recorded as $\left\{d_{C A N}^{b_{k}=\bar{b}_{k}^{M A P}}\right\}$. We note that there may only be $M / 2$ leaf candidates, when the fixed bit $b_{k}=\bar{b}_{k}^{M A P}$ is at the specific position in the range of $k \in\left\{\left(N_{w}-\right.\right.$ 2)BPS $\left.+1, \cdots,\left(N_{w}-1\right) \mathrm{BPS}\right\}$.

(3) Finally, the Max-Log-MAP of (20) may be revised for the Approx-Log-MAP as:

$L_{p}\left(b_{k}\right)= \begin{cases}\operatorname{jac}\left(-d_{C A N}\right)-\mathrm{jac}\left(-d_{C A N}^{b_{k}=\bar{b}_{k}^{M A P}}\right), & \text { if } b_{k}^{M A P}=1 \\ \mathrm{jac}\left(-d_{C A N}^{b_{k}=\bar{b}_{k}^{M A P}}\right)-\mathrm{jac}\left(-d_{C A N}\right), & \text { if } b_{k}^{M A P}=0 .\end{cases}$

We note that when the sizes of the two candidate groups $\left\{d_{C A N}\right\}$ and $\left\{d_{C A N}^{b_{k}=\bar{b}_{k}^{M A P}}\right\}$ are not the same, the size of the larger group may be reduced, so that ideally both groups disregard the same number of candidates. Ideally, any potential deviations introduced both by the jac $\left(-d_{C A N}\right)$ and by jac $\left(-d_{C A N}^{b_{k}=\bar{b}_{k}^{M A P}}\right)$ operations may be cancelled out. In practice, the SD's output candidates for $\left\{d_{C A N}\right\}$ and $\left\{d_{C A N}^{b_{k}=\bar{b}_{k}^{M A P}}\right\}$ are always constituted by either the sub-group of $M$ leaf candidates or by the sub-group of $M / 2$ leaf candidates. Therefore, for the larger-sized group, we may compare the best leaf candidates, which are supposed to have the minimum PED values in each sub-groups, and then we may delete the sub-groups associated with the highest locally best leaf candidate's PED value. For the example of Fig. 5, we may delete the sub-group of $\{20.05,24.09,46.615,35.05\}$, because the local best leaf candidates from each sub-group have the relationship of $20.05>14.91$. As a result, the Approx-Log-MAP of (31) may be implemented for the example seen in Figs. 1(a) and 5 as $L_{p}\left(b_{1}\right)=\mathrm{jac} \quad(-\{18.4,25.331,7.28,16.211\})-$ jac $(-\{25.118,35.09,18.97,14.91\})$.

One may argue that the SD does not visit all the MSDD candidates, which means that the group sizes of $\left\{d_{C A N}\right\}$ and $\left\{d_{C A N}^{b_{k}=\bar{b}_{k}^{M A P}}\right\}$ seen in (31) may be smaller than the group sizes of $\overline{\mathbf{S}}^{i} \in \overline{\mathbf{S}}_{b_{k}=1}$ and $\overline{\mathbf{S}}^{i} \in \overline{\mathbf{S}}_{b_{k}=0}$ seen in (16). In other words, ideally, the Approx-Log-MAP of (16) may include all the MSDD candidates, but naturally the SD may only visit a subset of them. Nonetheless, as suggested by [39], when the Approx-LogMAP corrects the difference between two probability metrics of $\left|d^{1}-d^{2}\right|$, only 8 values corresponding to $\left|d^{1}-d^{2}\right|$ ranging between 0 and 5.0 may be taken into account. This implies that large differences of $\left|d^{1}-d^{2}\right|>5.0$ are inherently ignored by the Approx-Log-MAP. Therefore, we may assume that the leaf candidates ignored by the SD may also be ignored by the Approx-Log-MAP, so that no extra complexity is imposed on the SD by our proposed Approx-Log-MAP.

We note that for a better implementation, Step (2) may be executed for all BPS $\left(N_{w}-1\right)$ fixed bits $\left\{b_{k}=\bar{b}_{k}^{M A P}\right\}_{k=1}^{\operatorname{BPS}\left(N_{w}-1\right)}$ before proceeding to Step (3), so that all the leaf nodes visited by the repeated SD searches may be utilized in Step (3). As a result, $\left\{d_{C A N}\right\}$ in (31) may be replaced by $\left\{d_{C A N}^{b_{k}^{C A N}}=b_{k}^{M A P}\right\}$, and then both $\left\{d_{C A N}^{b_{k}^{C A N}=b_{k}^{M A P}}\right\}$ and $\left\{d_{C A N}^{b_{k}=\bar{b}_{k}^{M A P}}\right\}$ in (31) may include all the leaf nodes obtained from Steps (1) and (2) corresponding to the specific bit $b_{k}$ being $b_{k}^{M A P}$ and $\bar{b}_{k}^{M A P}$, respectively. 
TABLE IV

Pseudo-Code for the Subfunctions of The Reduced-Complexity Soft-Decision MSDSD of TABle I, Where DPSK ( $M>4$ ) Is EmPloyed

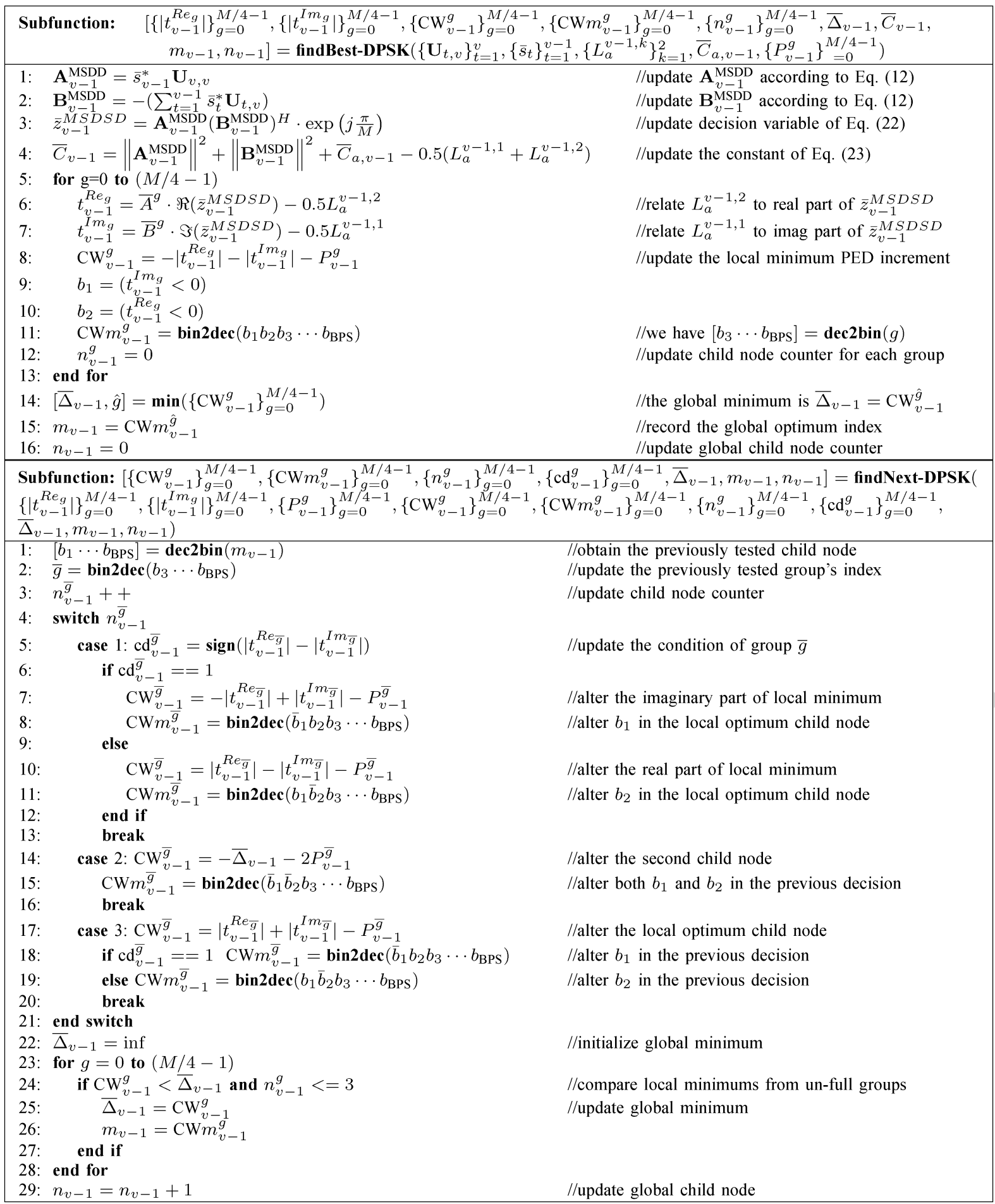

The Approx-Log-MAP may also be straightforwardly applied to the reduced-complexity soft-decision MSDSD of Section IV, where the simplified Schnorr-Euchner strategy of Tables II-IV can be invoked for all SD indice satisfying $v<N_{w}$. However, the original Schnorr-Euchner strategy of [3] has to be invoked for the specific SD index $v=N_{w}$, because all the leaf nodes at $v=N_{w}$ have to be recorded and produced for the Approx-Log-MAP.

\section{Performance Results}

\section{A. Approx-Log-MAP Versus Max-Log-MAP}

First of all, the accuracy of the extrinsic LLRs produced by the Approx-Log-MAP and Max-Log-MAP algorithms are tested as portrayed in Fig. 6, where the two PDFs $\left\{p\left(L_{e} \mid b\right)\right\}_{b=\{0,1\}}$ may be obtained by estimating the histograms of $L_{e}$, with the source bits being $b=\{0,1\}$. If the LLR 


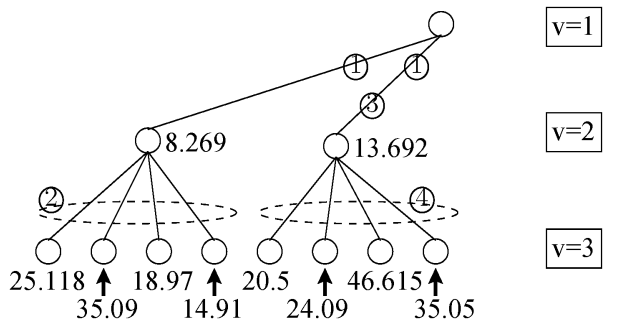

Fig. 5. Example of the soft-decision MSDSD conceived for DQPSK implementing Approx-Log-MAP, which invokes the SD seen in Fig. 1 with a fixed bit of $b_{1}=0$.

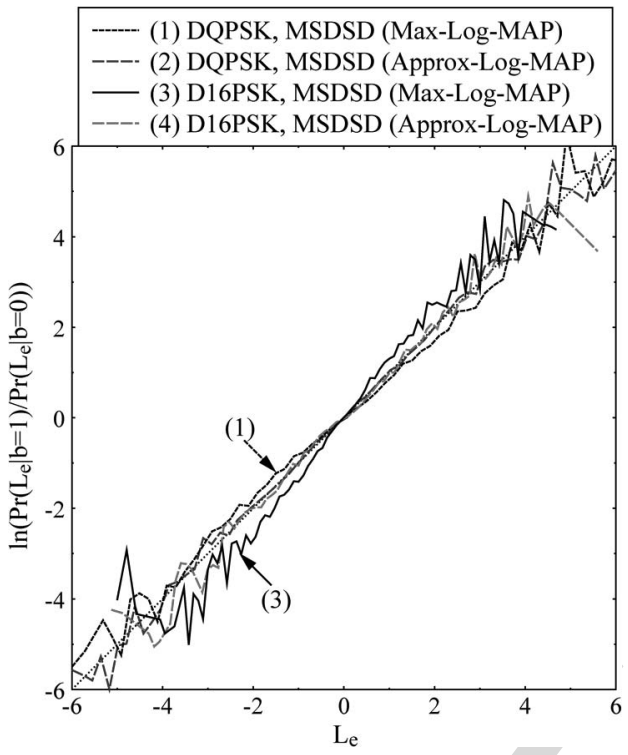

Fig. 6. LLR accuracy test for DQPSK and D16PSK employing both ApproxLog-MAP and Max-Log-MAP aided Subset MSDSD $\left(N_{w}=4\right)$, recorded at $\mathrm{SNR}=0 \mathrm{~dB}$ and $I_{A}=0$, where we have $N_{R}=2$ and $f_{d}=0.03$.

definition of $L_{e}=\ln \frac{p\left(L_{e} \mid b=1\right)}{p\left(L_{e} \mid b=0\right)}$ is statistically true, then the LLRs accuracy test is supposed to result in a diagonal line in Fig. 6. However, the LLRs of the Max-Log-MAP suffer from a noticeable deviation, which is effectively improved by the proposed Approx-Log-MAP, as evidenced by Fig. 6

It is worthy to note that the so-called Subset MSDSD is employed throughout this section. More explicitly, it was discovered in [44] that the symbols at the middle of the MSDSD window may be more reliably detected than those at its edges. Therefore, the Subset MSDSD overlaps the consecutive detection windows by $N_{O L}=3$ observations, so that the $\left(N_{O L}-\right.$ $1=2$ ) symbols detected at the edges may be discarded. ${ }^{5}$ The BER performance of Fig. 7 further confirms the advantage of the proposed Approx-Log-MAP algorithm in both TC coded and IRCC-URC coded DPSK systems, where the simulation parameters are summarized in Table V.

\section{B. Complexity Reduction}

To elaborate, the asymptotic complexity analysis of MSDSD was presented in [45] following the same guideline as the SD

${ }^{5}$ We note that the choice of $N_{O L}$ is independent of $N_{w}$, and it was demonstrated in [44] that increasing $N_{O L}$ beyond three does not provide any further advantage.

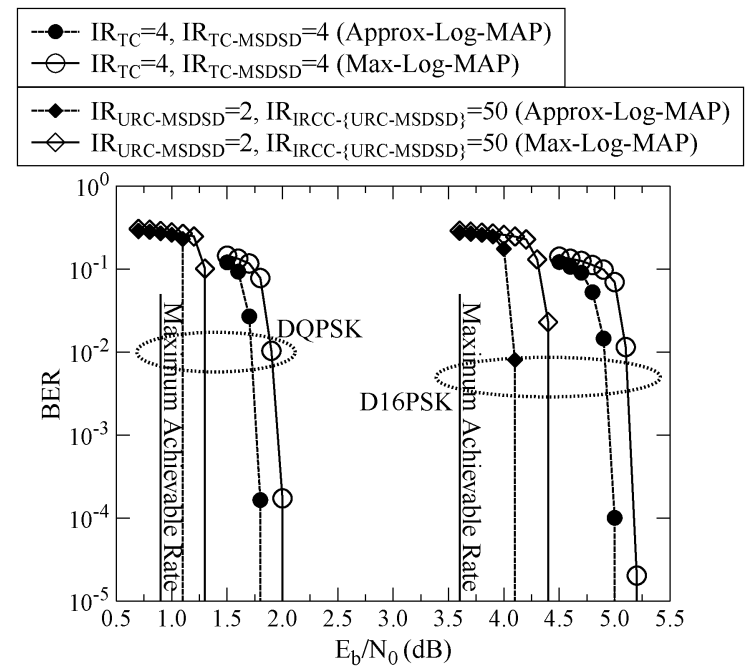

Fig. 7. BER performance of TC/IRCC-URC coded DPSK employing Subset $\operatorname{MSDSD}\left(N_{w}=4\right)$, where we have $N_{R}=2$ and $f_{d}=0.03$.

TABLE V

SYSTEM PARAMETERS

\begin{tabular}{|l|l|}
\hline $\begin{array}{l}\text { Channel } \\
\text { Coding }\end{array}$ & $\begin{array}{l}\text { TC coded DPSK (Schematics Fig. 3.1 in [38]) } \\
\text { IRCC-URC coded DPSK (Schematics Fig. 5.1 in [38]) }\end{array}$ \\
\hline TC coded & $I R_{T C}$ iterations within TC decoder; $I R_{T C}-M S D S D$ \\
DPSK & iterations between TC decoder and MSDSD \\
\hline IRCC-URC & $I R_{U R C-M S D S D}$ iterations between URC decoder and \\
coded & MSDSD; $I_{I R C C-\{U R C-M S D S D\}}$ iterations between \\
DPSK & IRCC and the amalgamated URC-MSDSD decoder. \\
\hline Frame length & 1000000 bits \\
\hline
\end{tabular}

TABLE VI

COMPLEXITY OF SOFT-DECISION MSDSD SUBFUNCTIONS

\begin{tabular}{|c|c|c|c|c|}
\hline & $\begin{array}{l}\text { real-valued mul- } \\
\text { tiplications }\end{array}$ & $\begin{array}{l}\text { real-valued } \\
\text { additions }\end{array}$ & comparisons & $\begin{array}{l}\text { visited } \\
\text { nodes }\end{array}$ \\
\hline sortDelta & $\begin{array}{r}\left(4 N_{R} v+\right. \\
\left.6 N_{R}\right) M\end{array}$ & $\begin{array}{r}\left(4 N_{R} v+\right. \\
\left.2 N_{R}\right) M\end{array}$ & $O(M \log M)$ & $M$ \\
\hline findBest-DBPSK & $4 N_{R} v+8 N_{R}$ & $4 N_{R} v+4 N_{R}$ & 2 & 1 \\
\hline findNext-DBPSK & 0 & 2 & 0 & 1 \\
\hline findBest-DQPSK & $\begin{array}{l}4 N_{R} v+8 N_{R}+ \\
7\end{array}$ & $\begin{array}{l}4 N_{R} v+4 N_{R}+ \\
6\end{array}$ & 4 & 1 \\
\hline findNext-DQPSK & $\leq 1$ & $\leq 4$ & $\leq 3$ & 1 \\
\hline findBest-DPSK & $\begin{array}{l}4 N_{R} v+8 N_{R}+ \\
M+4\end{array}$ & $\begin{array}{c}4 N_{R} v+4 N_{R}+ \\
3 M / 2+2\end{array}$ & $5 M / 4$ & $M / 4$ \\
\hline findNext-DPSK & $\leq 3$ & $\leq 7$ & $\leq M / 2+2$ & 1 \\
\hline
\end{tabular}

aided BLAST of [18], which was only feasible when invoking the sub-optimal Fincke-Pohst strategy of [21]. By contrast, in this work, we focus our attention on the complexity reduction achieved for the optimal Schnorr-Euchner strategy of [1]. Against this background, the detection complexities of the softdecision MSDSD subfunctions are summarized in Table VI, ${ }^{6}$ where "sortDelta" refers to the conventional Schnorr-Euchner search strategy in [3], while the rest of the proposed subfunctions are given by Tables II-IV. It can be seen in Table VI that the proposed Schnorr-Euchner search strategy visits a reduced

\footnotetext{
${ }^{6}$ We note that unneccessary calculations are eliminated for Table VI. For example, both $\left\{0.5 L_{a}^{v-1,1}\right\}_{\forall v}$ and $\left\{0.5 L_{a}^{v-1,2}\right\}_{\forall v}$ may be calculated before invoking the MSDSD's subfunctions. Furthermore, the function of $\left[b_{1} \cdots\right.$ $\left.b_{\mathrm{BPS}}\right]=\mathbf{d e c} 2 \mathbf{b i n}(m)$ may be implemented by a pre-stored lookup table for bit-mapping, while its inverse function $m=\operatorname{bin} 2 \operatorname{dec}\left(b_{1} \cdots b_{\mathrm{BPS}}\right)=b_{1}$. $2^{\mathrm{BPS}-1}+b_{2} \cdot 2^{\mathrm{BPS}-2}+\cdots+b_{\mathrm{BPS}-1} \cdot 2+b_{\mathrm{BPS}}$ may require a total number of $(B P S-1)$ multiplications as well as $(B P S-1)$ additions.
} 


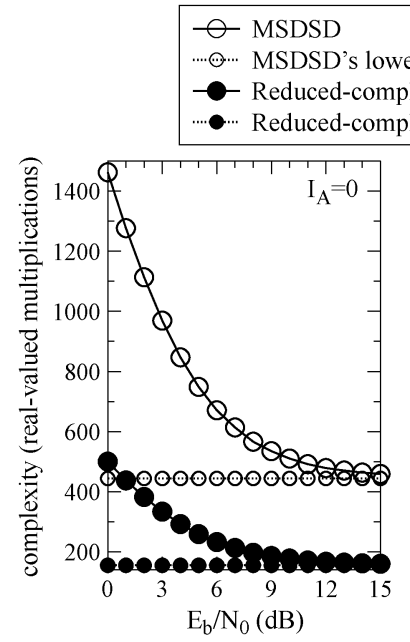

(a)
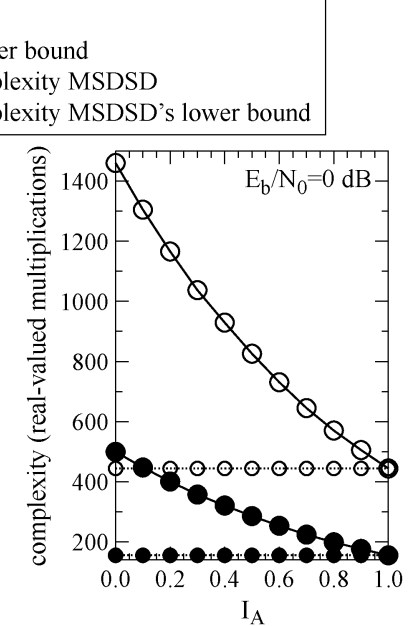

(b)
Fig. 8. Complexity (number of real-valued multiplications) comparison between the conventional MSDSD algorithm of [3] associated with $N_{w}=4$ and the reduced-complexity MSDSD algorithm of Tables I and III for coded DQPSK, where we have $N_{R}=2$ and $f_{d}=0.03$. (a) $E_{b} / N_{0}=0 \sim 15 \mathrm{~dB}$. (b) $I_{A}=0 \sim 1$.

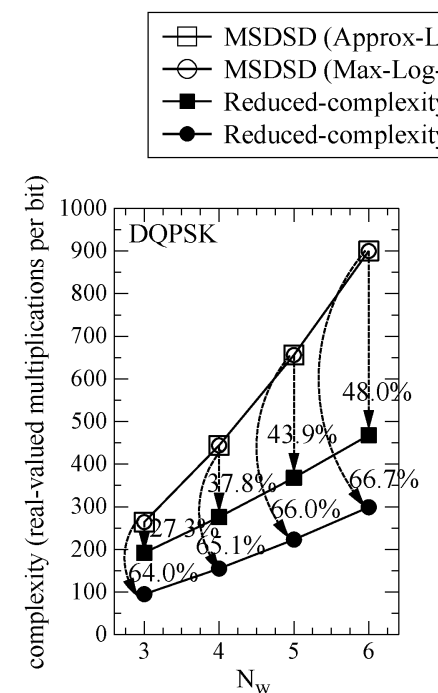

(a)

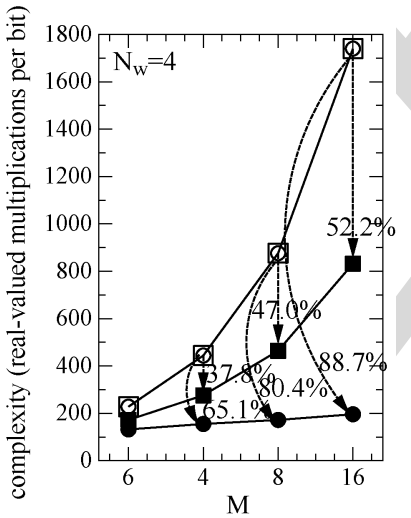

(b)
Fig. 9. Complexity-Reduction Ratio (CRR) achieved by the reducedcomplexity MSDSD algorithm of Table I compared to the conventional MSDSD algorithm of [3] recorded at $E_{b} / N_{0}=0 \mathrm{~dB}$ and $I_{A}=1$, where we have $N_{R}=2$ and $f_{d}=0.03$. (a) $N_{w}=\{3,4,5,6\}$. (b) $M=\{2,4,8,16\}$.

number of nodes, which results in a reduced complexity in all categories.

The complexity of the conventional MSDSD algorithm and that of the proposed MSDSD algorithm conceived for coded DQPSK are compared in Fig. 8 in terms of the total number of real-valued multiplications. It is confirmed by Fig. 8(a) and (b) that the complexities of the MSDSD algorithms may converge to their lower bounds, as $E_{b} / N_{0}$ and/or $I_{A}$ increase. Fig. 8 demonstrates that the proposed MSDSD of Table I substantially reduces the complexity of the conventional MSDSD in [3]. The Complexity-Reduction Ratios (CRRs) achieved by our reduced-complexity design are further presented in Fig. 9.
We note that for the conventional MSDSD, the Approx-LogMAP and the Max-Log-MAP impose the same number of realvalued multiplications. By contrast, for the proposed MSDSD, the Approx-Log-MAP has to invoke the conventional SchnorrEuchner strategy for the specific SD index $v=N_{w}$, which results in a higher number of multiplications than the Max-LogMAP. Nonetheless, Fig. 9(a) and (b) demonstrate that substantial complexity reductions of up to $C R R=48.0 \%$ and $C R R=$ $52.2 \%$ are achieved by the Subset MSDSD $\left(N_{w}=6\right)$ aided DQPSK and by the Subset MSDSD $\left(N_{w}=4\right)$ aided D16PSK, when the Approx-Log-MAP is implemented. Furthermore, even more substantial complexity reductions of $C R R=66.7 \%$ and $C R R=88.7 \%$ are achieved by the Subset MSDSD $\left(N_{w}=\right.$ 6) aided DQPSK and by the Subset MSDSD $\left(N_{w}=4\right)$ aided D16PSK, when the Max-Log-MAP is implemented. We note that the complexity reductions achieved by the proposed MSDSD are especially significant, when the MSDSD is iteratively invoked several times by the turbo detected systems.

Once again, we note that the proposed soft-decision MSDSD algorithm presented in Tables I-IV has exactly the same detection capability as the conventional soft-decision MSDSD algorithm presented in Appendix I of [3]. We have arranged for them to decode the same channel output associated with the same $a$ priori soft input, and we found that they always produce exactly the same SD decisions.

\section{Coherent Versus Noncoherent}

Last but not least, MSDSD aided DQPSK is compared to its PSAM aided coherent QPSK counterpart. First of all, Fig. $10^{7}$ demonstrates that when we have $f_{d}=0.03$, the LLRs produced by the PSAM aided QPSK detector suffer from severe deviation from the true probabilities, which may result in disproportionately high LLR values that may misinform the channel decoder.

Secondly, Fig. $11^{8}$ demonstrates that when we have $f_{d}=0.001$, the coherent PSAM aided QPSK significantly outperforms the Subset MSDSD aided DQPSK in both our TC and IRCC-URC coded systems. However, when the normalized Doppler frequency is increased to $f_{d}=0.03$, the PSAM aided QPSK's performance degrades substantially, while the Subset MSDSD aided DQPSK only suffers from a small BER performance degradation, which gives the noncoherent schemes a $0.7 \mathrm{~dB}$ and a $1.4 \mathrm{~dB}$ performance advantage over their coherent counterparts in the context of our TC and IRCC-URC coded systems, respectively, as evidenced by Fig. 11. Therefore, we may conclude that the DPSK schemes employing MSDSD may be deemed to be a more suitable candidate for channel coded systems operating at high Doppler frequencies.

\footnotetext{
${ }^{7}$ When we have $f_{d}=0.03$, the PSAM's pilot spacing $N_{P S}$ is reduced to 12 in order to sample the channel more frequently, while the PSAM's observation window length $N_{O W}$ (number of filter coefficients) is also reduced to 12 , due to the weak temporal correlation.

${ }^{8}$ There is no iteration between the QPSK detector and the channel decoder in Fig. 11, because the QPSK detection does not produce any iteration gain [28]. Nonetheless, the coded coherent schemes and their noncoherent counterparts have the same total number of iterations for turbo detection.
} 


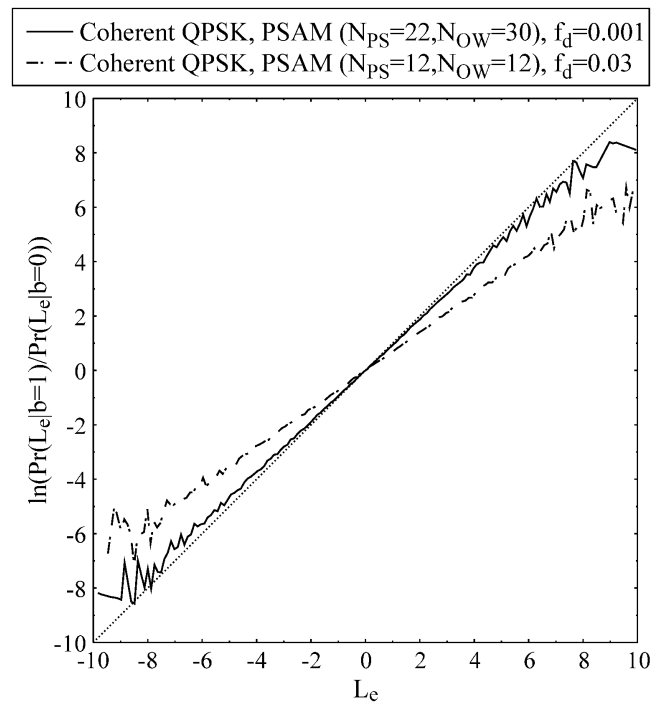

Fig. 10. LLR accuracy test for PSAM [11] aided coherent QPSK, recorded at $\mathrm{SNR}=0 \mathrm{~dB}$ and $I_{A}=0$, where we have $N_{R}=2$.

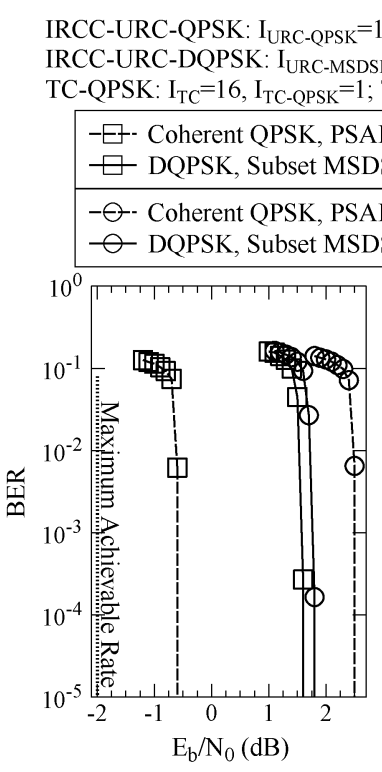

(a)
$=1, \mathrm{I}_{\text {IRCC- }\{\text { URC-QPSK }\}}=60$

$2, \mathrm{I}_{\text {IRCC- }\{\text { URC-MSDSD }\}}=30$ -DQPSK: $\mathrm{I}_{\mathrm{TC}}=4, \mathrm{I}_{\mathrm{TC}-\mathrm{MSDSD}}=4$

Fig. 11. BER performance comparison between TC/IRCC-URC coded PSAM [11] aided coherent QPSK and TC/IRCC-URC coded Subset MSDSD aided DQPSK, where the Approx-Log-MAP is invoked, while $N_{R}=2$ receive antennas are used. (a) TC coded systems. (b) IRCC-URC coded systems.

\section{CONCLUSION}

In this paper, the Schnorr-Euchner search strategy was configured to always visit the MPSK constellation points in a zigzag fashion both for the hard-decision MSDSD of Section II and for the soft-decision MSDSD of Section IV. The complexity results of Figs. 8 and 9 demonstrated that our proposed design offers a substantial complexity reduction. Furthermore, the Approx-Log-MAP algorithm implemented by the MSDSD was proposed in Section V, which improved the Max-LogMAP, as demonstrated by Figs. 6 and 7. Finally, our comparison of the channel-coded coherent and noncoherent schemes characterized in Figs. 10 and 11 suggested that the DPSK schemes employing MSDSD may be deemed to be more suitable candidates for channel coded systems operating at high Doppler frequencies.

\section{APPENDIX}

In this section, we aim to conceive the SD aided V-BLAST employing MPSK [29]-[34] in the same form as the MSDSD aided DPSK, so that the reduced-complexity Schnorr-Euchner search strategy proposed in Section IV and the Approx-LogMAP proposed in Section $V$ may also be applied to the V-BLAST detection.

Explicitly, the $\left(1 \times N_{T}\right)$-element V-BLAST transmission matrix is given by:

$$
\mathbf{S}=\left[s_{1}, \quad \cdots, \quad s_{N_{T}}\right]=\left[\begin{array}{lll}
\frac{s^{m_{1}}}{\sqrt{N_{T}}}, & \cdots, & \frac{s^{m_{N_{T}}}}{\sqrt{N_{T}}}
\end{array}\right],
$$

where the MPSK/QAM symbols are separately modulated as $\left\{s^{m_{v}}\right\}_{v=1}^{N_{T}}$, while the factor $\sqrt{N_{T}}$ normalizes the transmission power. The signal received by the $N_{R}$ antenna elements at the receiver may be modelled as:

$$
\mathbf{Y}=\mathbf{S H}+\mathbf{V}
$$

where the $\left(1 \times N_{T}\right)$-element vector $\mathbf{S}$ and the $\left(1 \times N_{R}\right)$-element vector $\mathbf{Y}$ represent the input and output signals of the MIMO channels, respectively. Furthermore, the $\left(N_{T} \times N_{R}\right)$-element $\mathbf{H}$ matrix of (33) models the MIMO's Rayleigh fading channels, while the $\left(1 \times N_{R}\right)$-element AWGN vector $\mathbf{V}$ of (33) models the zero-mean Gaussian random variables with a common complex variance of $N_{0}$, whose PDF is given by $p(\mathbf{V})=p\left(\mathbf{Y} \mid \mathbf{S}^{i}\right)=$ $\frac{1}{\left(\pi N_{0}\right)^{N_{R} N_{P}}} \exp \left(-\frac{\left\|\mathbf{Y}-\mathbf{S}^{i} \mathbf{H}\right\|^{2}}{N_{0}}\right)$, where there is a total number of $M^{N_{T}}$ combinations $\left\{\mathbf{S}^{i}\right\}_{i=0}^{M^{N_{T}}-1}$ for the MIMO transmission matrix $\mathbf{S}$ in (33). In order to invoke the classic SD, the V-BLAST receiver may apply QR decomposition to $\mathbf{H}^{H}$ [31]-[34], which results in $\mathbf{H}=(\mathbf{Q U})^{H}=\mathbf{L} \mathbf{Q}^{H}$, where the $\left(N_{R} \times N_{T}\right)$-element matrix $\mathbf{Q}$ has orthogonal columns $\mathbf{Q}^{H} \mathbf{Q}=I_{N_{T}}$, while $\mathbf{U}$ and $\mathbf{L}=\mathbf{U}^{H}$ are upper- and lower- triangular matrices, respectively. As a result, (33) may be reformulated as:

$$
\tilde{\mathbf{Y}}=\mathbf{Y Q}=\mathbf{S L}+\mathbf{V Q},
$$

where VQ has exactly the same statistics as the AWGN vector $\mathbf{V}$. Based on the conditional probability $p\left(\mathbf{Y} \mid \mathbf{S}^{i}\right)$ as well as on the Bayes's theorem of (5), the ED to be minimized by the SD may be expressed as [34], [40]:

$$
d=\frac{\sum_{v=1}^{N_{T}}\left|\widetilde{Y}_{v}-\sum_{t=v}^{N_{T}} l_{t, v} s_{t}\right|^{2}}{N_{0}}-\sum_{v=1}^{N_{T}} \sum_{\bar{k}_{v}=1}^{\text {BPS }}\left[\widetilde{b}_{\bar{k}_{v}} L_{a}\left(b_{\bar{k}_{v}}\right)-\bar{C}_{a, \bar{k}_{v}}^{S D}\right]
$$

where an extra constant $\bar{C}_{a, \bar{k}_{v}}^{S D}=\frac{1}{2}\left[\left|L_{a}\left(b_{\bar{k}_{v}}\right)\right|+L_{a}\left(b_{\bar{k}_{v}}\right)\right]$ is introduced in order to guarantee that the ED remains non-negative [34], [40]. According to the ED of (35), the SD's PED may be 
formulated as $d_{v}=d_{v+1}+\Delta_{v}$, where the PED increment $\Delta_{v}$ may be expressed as:

$$
\begin{aligned}
\Delta_{v} & =\frac{\left|\widetilde{Y}_{v}-\sum_{t=v}^{N_{T}} l_{t, v} s_{t}\right|^{2}}{N_{0}}-\sum_{\bar{k}_{v}=1}^{\mathrm{BPS}}\left[\widetilde{b}_{\bar{k}_{v}} L_{a}\left(b_{\bar{k}_{v}}\right)-\bar{C}_{a, \bar{k}_{v}}^{S D}\right] \\
& =\left|\widetilde{A}_{v}^{S D}-s_{v}^{m} \widetilde{B}_{v}^{S D}\right|^{2}-\sum_{\bar{k}_{v}=1}^{\mathrm{BPS}}\left[\widetilde{b}_{\bar{k}_{v}} L_{a}\left(b_{\bar{k}_{v}}\right)-\bar{C}_{a, \bar{k}_{v}}^{S D}\right],
\end{aligned}
$$

where we have $\widetilde{A}_{v}^{S D}=\frac{\widetilde{Y}_{v}-\sum_{t=v+1}^{N_{T}} l_{t, v} S_{t}}{\sqrt{N_{0}}}$ and $\widetilde{B}_{v}^{S D}=\frac{l_{v, v}}{\sqrt{N_{0} N_{T}}}$. As a result, (36) is in the same form as the MSDSD's PED increment of (19) in Section III and (21) in Section IV. Therefore, the reduced-complexity Schnorr-Euchner search strategy and the proposed Approx-Log-MAP solution may be directly applied to the SD aided V-BLAST employing MPSK.

\section{REFERENCES}

[1] C. P. Schnorr and M. Euchner, "Lattice basis reduction: Improved practical algorithms and solving subset sum problems," Math. Programm., vol. 66, no. 1-3, pp. 181-191, Aug. 1994.

[2] L. Lampe, R. Schober, V. Pauli, and C. Windpassinger, "Multiple-symbol differential sphere decoding," IEEE Trans. Commun., vol. 53, no. 12, pp. 1981-1985, Dec. 2005.

[3] V. Pauli, L. Lampe, and R. Schober, "Turbo DPSK' using soft multiplesymbol differential sphere decoding," IEEE Trans. Inf. Theory, vol. 52, no. 4, pp. 1385-1398, Apr. 2006.

[4] J. Lawton, "Theoretical error rates of differentially coherent binary and kineplex data transmission systems," Proc. Inst. Radio Eng., vol. 47, no. 2, pp. 333-334, 1959.

[5] J. Lawton, "Investigation of digital data communication systems," Cornell Aeron. Lab., Buffalo, NY, USA, 1961.

[6] C. Cahn, "Performance of digital phase-modulation communication systems," IRE Trans. Commun. Syst., vol. 7, no. 1, pp. 3-6, Jan. 1959.

[7] P. Bello and B. D. Nelin, "The influence of fading spectrum on the binary error probabilites of incoherent and differentially coherent matched filter recievers," IRE Trans. Commun. Syst., vol. 10, no. 2, pp. 160-168, Jun. 1962.

[8] D. Divsalar and M. K. Simon, "Multiple-symbol differential detection of MPSK," IEEE Trans. Commun., vol. 38, no. 3, pp. 300-308, Mar. 1990.

[9] P. Ho and D. Fung, "Error performance of multiple-symbol differential detection of PSK signals transmitted over correlated Rayleigh fading channels," IEEE Trans. Commun., vol. 40, no. 10, pp. 1566-1569, Oct. 1992.

[10] K. Mackenthun, "A fast algorithm for multiple-symbol differential detection of MPSK," IEEE Trans. Commun., vol. 42, no. 2-4, pp. 1471-1474, Feb.-Apr. 1994.

[11] J. Cavers, "An analysis of pilot symbol assisted modulation for Rayleigh fading channels," IEEE Trans. Veh. Technol., vol. 40, no. 4, pp. 686-693, Nov. 1991.

[12] H. Leib and S. Pasupathy, "The phase of a vector perturbed by Gaussian noise and differentially coherent receivers," IEEE Trans. Inf. Theory, vol. 34, no. 6, pp. 1491-1501, Nov. 1988.

[13] H. Leib, "Data-aided noncoherent demodulation of DPSK," IEEE Trans. Commun., vol. 43, no. 2-4, pp. 722-725, Feb.-Apr. 1995.

[14] R. Schober, W. Gerstacker, and J. Huber, "Decision-feedback differential detection of MDPSK for flat Rayleigh fading channels," IEEE Trans. Commun., vol. 47, no. 7, pp. 1025-1035, Jul. 1999.

[15] E. Agrell, T. Eriksson, A. Vardy, and K. Zeger, "Closest point search in lattices," IEEE Trans. Inf. Theory, vol. 48, no. 8, pp. 2201-2214, Aug. 2002.

[16] C. Berrou and A. Glavieux, "Near optimum error correcting coding and decoding: Turbo-codes," IEEE Trans. Commun., vol. 44, no. 10, pp. 1261-1271, Oct. 1996.

[17] M. El-Hajjar and L. Hanzo, "EXIT charts for system design and analysis," IEEE Commun. Surveys Tuts., vol. 16, pp. 127-153, 1st Quart. 2014.

[18] J. Jalden and B. Ottersten, "On the complexity of sphere decoding in digital communications," IEEE Trans. Signal Process., vol. 53, no. 4, pp. 1474-1484, Apr. 2005.

[19] B. Hassibi and H. Vikalo, "On the sphere-decoding algorithm I. Expected complexity," IEEE Trans. Signal Process., vol. 53, no. 8, pp. 2806-2818, Aug. 2005
[20] H. Vikalo and B. Hassibi, "On the sphere-decoding algorithm II. Generalizations, second-order statistics, and applications to communications," IEEE Trans. Signal Process., vol. 53, no. 8, pp. 2819-2834, Aug. 2005

[21] U. Fincke and M. Pohst, "Improved methods for calculating vectors of short length in a lattice, including a complexity analysis," Math. Comput., vol. 44, no. 170, pp. 463-471, 1985.

[22] A. Kyrillidis and G. N. Karystinos, "Fixed-rank Rayleigh quotient maximization by an MPSK sequence," IEEE Trans. Commun., vol. 62, no. 3 , pp. 961-975, Mar. 2014.

[23] Z. Pi and F. Khan, "An introduction to millimeter-wave mobile broadband systems," IEEE Commun. Mag., vol. 49, no. 6, pp. 101-107, Jun. 2011.

[24] T. Rappaport, J. Murdock, and F. Gutierrez, "State of the art in 60-GHz integrated circuits and systems for wireless communications," Proc. IEEE, vol. 99, no. 8, pp. 1390-1436, Aug. 2011.

[25] T. Marzetta, "Noncooperative cellular wireless with unlimited numbers of base station antennas," IEEE Trans. Wireless Commun., vol. 9, no. 11, pp. 3590-3600, Nov. 2010 .

[26] F. Rusek et al., "Scaling up MIMO: Opportunities and challenges with very large arrays," IEEE Signal Process. Mag., vol. 30, no. 1, pp. 40-60, Jan. 2013.

[27] C. Xu, S. Sugiura, S. X. Ng, and L. Hanzo, "Reduced-complexity noncoherently detected differential space-time shift keying," IEEE Signal Process. Lett., vol. 18, no. 3, pp. 153-156, Mar. 2011.

[28] C. Xu, D. Liang, S. Sugiura, S. X. Ng, and L. Hanzo, "Reduced-complexity approx-log-MAP and max-log-MAP soft PSK/QAM detection algorithms," IEEE Trans. Commun., vol. 61, no. 4, pp. 1415-1425, Apr. 2013.

[29] O. Damen, A. Chkeif, and J. C. Belfiore, "Lattice code decoder for spacetime codes," IEEE Commun. Lett., vol. 4, no. 5, pp. 161-163, May 2000.

[30] M. Damen, H. El-Gamal, and G. Caire, "On maximum-likelihood detection and the search for the closest lattice point," IEEE Trans. Inf. Theory, vol. 49 , no. 10 , pp. 2389-2402, Oct. 2003.

[31] B. Hochwald and S. ten Brink, "Achieving near-capacity on a multipleantenna channel," IEEE Trans. Commun., vol. 51, no. 3, pp. 389-399, Mar. 2003.

[32] H. Vikalo, B. Hassibi, and T. Kailath, "Iterative decoding for MIMO channels via modified sphere decoding," IEEE Trans. Wireless Commun., vol. 3, no. 6, pp. 2299-2311, Jan. 2004.

[33] C. Studer, A. Burg, and H. Bolcskei, "Soft-output sphere decoding: Algorithms and VLSI implementation," IEEE J. Sel. Areas Commun., vol. 26, no. 2, pp. 290-300, Feb. 2008.

[34] C. Studer and H. Bolcskei, "Soft-input soft-output single tree-search sphere decoding," IEEE Trans. Inf. Theory, vol. 56, no. 10, pp. 48274842, Sep. 2010

[35] D. Divsalar and M. Simon, "Maximum-likelihood differential detection of uncoded and trellis coded amplitude phase modulation over AWGN and fading channels-Metrics and performance," IEEE Trans. Commun., vol. 42, no. 1, pp. 76-89, Jan. 1994.

[36] R. H. Clarke, "A statistical theory of mobile radio reception," Bell Labs Tech. J., vol. 47, no. 6, pp. 957-1000, Jul./Aug. 1968.

[37] W. Koch and A. Baier, "Optimum and sub-optimum detection of coded data disturbed by time-varying intersymbol interference," in Proc. IEEE GLOBECOM, San Diego, CA, USA, Dec. 1990, vol. 3, pp. 1679-1684.

[38] L. Hanzo, O. Alamri, M. El-Hajjar, and N. Wu, Near-Capacity MultiFunctional MIMO Systems: Sphere-Packing, Iterative Detection and Cooperation. Hoboken, NJ, USA: Wiley, May 2009.

[39] P. Robertson, E. Villebrun, and P. Hoeher, "A comparison of optimal and sub-optimal MAP decoding algorithms operating in the log domain," in Proc. IEEE ICC, Seattle, WA, USA, Jun. 1995, vol. 2, pp. 1009-1013.

[40] S. Baro, J. Hagenauer, and M. Witzke, "Iterative detection of MIMO transmission using a List-Sequential (LISS) detector," in Proc. IEEE ICC, May 2003, vol. 4, pp. 2653-2657.

[41] J. Jalden and B. Ottersten, "Parallel implementation of a soft output sphere decoder," in Proc. 39th Asilomar Conf. Signals, Syst. Comput., Oct. 2005, pp. 581-585.

[42] T. H. Cormen, C. E. Leiserson, R. L. Rivest, and C. Stein, Introduction to Algorithms. Cambridge, MA, USA: MIT Press, 2001.

[43] M. A. Bender, M. Farach-Colton, and M. Mosteiro, "Insertion sort is O(n $\log$ n)," Theory Comput. Syst., vol. 39, no. 3, pp. 391-397, 2006.

[44] V. Pauli and L. Lampe, "Tree-search multiple-symbol differential decoding for unitary space-time modulation," IEEE Trans. Commun., vol. 55, no. 8, pp. 1567-1576, Aug. 2007.

[45] V. Pauli and L. Lampe, "On the complexity of sphere decoding for differential detection," IEEE Trans. Inf. Theory, vol. 53, no. 4, pp. 1595-1603, Apr. 2007. 


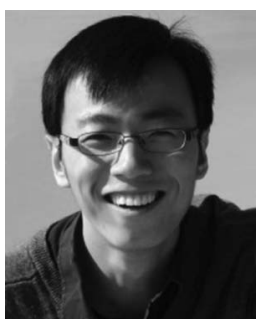

Chao Xu (S'09-M'14) received the B.Eng. degree from Beijing University of Posts and Telecommunications, Beijing, China, and the B.Sc. (Eng.) with First Class Honours from Queen Mary, University of London, London, U.K., through a Sino-U.K. joint degree program in 2008 , both in telecommunications engineering with management. He received the M.Sc. degree with distinction in Radio Frequency Communication Systems and the Ph.D. degree in wireless communications from the University of Southampton, Southampton, U.K., in 2009 and 2015, respectively. He is currently a Postdoctoral Researcher working at Southampton Wireless Group, University of Southampton, Southampton, U.K. His research interests include reduced-complexity multiple-input multiple-output design, noncoherent detection, extrinsic-information-transfer-chart-aided turbo detection, and cooperative communications. He was awarded the Best M.Sc. Student in Broadband and Mobile Communication Networks by the IEEE Communications Society (United Kingdom and Republic of Ireland Chapter) in 2009, and he received 2012 Chinese Government Award for Outstanding Self-Financed Student Abroad.

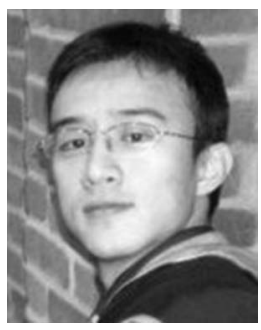

Xin Zuo received the B.Eng. degree in telecommunication engineering from the Beijing University of Posts and Telecommunications (BUPT), Beijing in 2008, and the M.Sc. degree with distinction in radio frequency communication systems from the University of Southampton, Southampton, U.K., in 2009. $\mathrm{He}$ is currently working towards the Ph.D. degree in the Wireless Communications Group in the University of Southampton. His research interests include the error-tolerant design and the implementation of LDPC decoders.

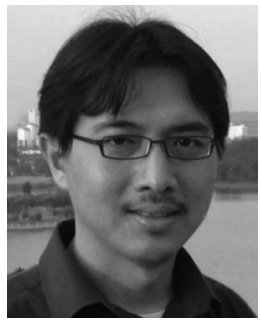

Soon Xin Ng (S'99-M'03-SM'08) received the B.Eng. degree (First Class) in electronic engineering and the $\mathrm{Ph} . \mathrm{D}$. degree in telecommunications from the University of Southampton, Southampton, U.K., in 1999 and 2002, respectively. From 2003 to 2006, he was a Postdoctoral Research Fellow working on collaborative European research projects known as SCOUT, NEWCOM, and PHOENIX. Since August 2006, he has been a member of the academic staff in the School of Electronics and Computer Science, University of Southampton. He is involved in the OPTIMIX and CONCERTO European projects as well as the IU-ATC and UC4G projects. He is currently an Associate Professor in telecommunications at the University of Southampton.

His research interests include adaptive coded modulation, coded modulation, channel coding, space-time coding, joint source and channel coding, iterative detection, OFDM, MIMO, cooperative communications, distributed coding, quantum error correction codes and joint wireless-and-optical-fibre communications. He has published over 180 papers and co-authored two John Wiley/IEEE Press books in this field. He is a Chartered Engineer and a Fellow of the Higher Education Academy in the U.K.

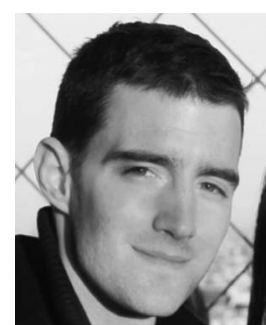

Robert G. Maunder (S'03-M'08-SM'12) received the B.Eng. degree (first class honors) in electronic engineering and the Ph.D. degree in wireless communications from University of Southampton, U.K., in 2003 and 2007, respectively. He became a Lecturer in 2007 and an Associated Professor in 2013. His research interests include joint source/ channel coding, iterative decoding, irregular coding and modulation techniques. For further information on this research, please refer to http://users.ecs.soton. ac.uk/rm

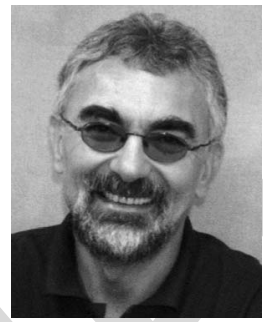

Lajos Hanzo (M'91-SM'92-F'04) received the degree in electronics in 1976 and the doctorate in 1983. In 2009 he was awarded an honorary doctorate by the Technical University of Budapest, while in 2015 by the University of Edinburgh. During his 38-year career in telecommunications he has held various research and academic posts in Hungary, Germany and the U.K. Since 1986, he has been with the School of Electronics and Computer Science, University of Southampton, U.K., where he holds the chair in telecommunications. He has successfully supervised about $100 \mathrm{Ph}$.D. students, co-authored 20 John Wiley/IEEE Press books on mobile radio communications totalling in excess of 10,000 pages, published $1400+$ research entries at IEEE Xplore, acted both as TPC and General Chair of IEEE conferences, presented keynote lectures, and been awarded a number of distinctions. Currently, he is directing a 60-strong academic research team, working on a range of research projects in the field of wireless multimedia communications sponsored by industry, the Engineering and Physical Sciences Research Council (EPSRC) U.K., the European Research Council's Advanced Fellow Grant and the Royal Society's Wolfson Research Merit Award. He is an enthusiastic supporter of industrial and academic liaison and he offers a range of industrial courses. He is also a Governor of the IEEE Vehicular Technology Society. During 2008-2012, he was the Editor-in-Chief of the IEEE Press and a Chaired Professor also at Tsinghua University, Beijing. His research is funded by the European Research Council's Senior Research Fellow Grant. He has $22000+$ citations. For further information on research in progress and associated publications please refer to http://www-mobile.ecs.soton.ac.uk 